\title{
At risk for psychosis : exploring cognition as intermediate phenotype
}

Citation for published version (APA):

Pfeifer, S. (2011). At risk for psychosis : exploring cognition as intermediate phenotype. [Doctoral Thesis, Maastricht University]. Maastricht University. https://doi.org/10.26481/dis.20110217sp

Document status and date:

Published: 01/01/2011

DOI:

10.26481/dis.20110217sp

Document Version:

Publisher's PDF, also known as Version of record

\section{Please check the document version of this publication:}

- A submitted manuscript is the version of the article upon submission and before peer-review. There can be important differences between the submitted version and the official published version of record.

People interested in the research are advised to contact the author for the final version of the publication, or visit the DOI to the publisher's website.

- The final author version and the galley proof are versions of the publication after peer review.

- The final published version features the final layout of the paper including the volume, issue and page numbers.

Link to publication

\footnotetext{
General rights rights.

- You may freely distribute the URL identifying the publication in the public portal. please follow below link for the End User Agreement:

www.umlib.nl/taverne-license

Take down policy

If you believe that this document breaches copyright please contact us at:

repository@maastrichtuniversity.nl

providing details and we will investigate your claim.
}

Copyright and moral rights for the publications made accessible in the public portal are retained by the authors and/or other copyright owners and it is a condition of accessing publications that users recognise and abide by the legal requirements associated with these

- Users may download and print one copy of any publication from the public portal for the purpose of private study or research.

- You may not further distribute the material or use it for any profit-making activity or commercial gain

If the publication is distributed under the terms of Article $25 \mathrm{fa}$ of the Dutch Copyright Act, indicated by the "Taverne" license above, 


\section{AT RISK FOR PSYCHOSIS}

Exploring Cognition as Intermediate Phenotype 
Colophon

ISBN | 978-90-5335-359-2

Graphic Design | Stefanie Pfeifer, Andreas Zimmerer Print | Ridderprint, Ridderkerk

Copyright @ S. Pfeifer, Maastricht 2011

All rights reserved. No part of this publication may be reproduced, stored in a retrieval system, or transmitted, in any form or by any means, electronic, mechanical, photocopying, recording, or otherwise, without the prior permission in writing from the proprietor. 


\section{AT RISK FOR PSYCHOSIS}

\section{Exploring Cognition as Intermediate Phenotype}

\section{PROEFSCHRIFT}

Ter verkrijging van de graad van doctor aan de Universiteit Maastricht, op gezag van de Rector Magnificus, Prof. mr. G.P.M.F. Mols, volgens het besluit van het College van Decanen, in het openbaar te verdedigen op donderdag 17. februari 2011 om 12:00 uur

door

\section{Stefanie Pfeifer}

Geboren op 11 oktober 1975 te Augsburg 


\section{Promotores}

Prof. dr. J. van Os

Prof. dr. N.O. Schiller

\section{Copromotor}

Dr. L. Krabbendam (Vrije Universiteit Amsterdam)

\section{Beoordelingscommissie}

Prof. dr. M. De Vries (voorzitter)

Prof. dr. A. Aleman (UMC Groningen)

Dr. L. Jonkman

Prof. dr. D. Linszen (Universiteit van Amsterdam)

Dr. M. Marcelis

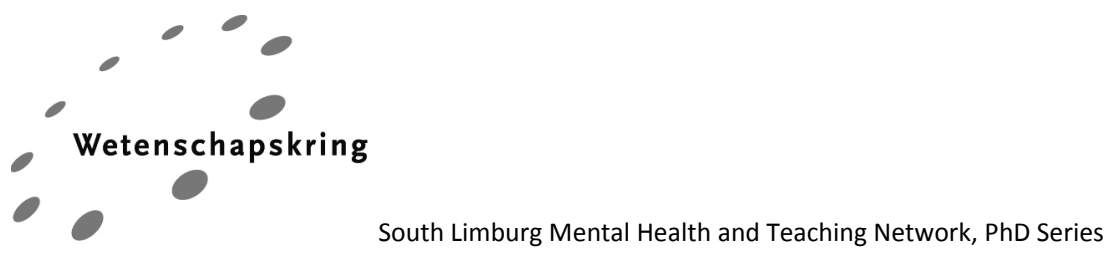

The research presented in this thesis was conducted at the School for Mental Health and Neuroscience, Department of Psychiatry and Neuropsychology of Maastricht University, Maastricht University Medical Centre and Mondriaan Zorggroep.

Publication of this thesis was financially supported by: Lundbeck B.V. 
Für meine Eltern 


\section{Paranimfen}

Tineke Lataster

Christine Firk 
CONTENTS

Chapter $1 \quad$ Introduction

Chapter 2 A cognitive intermediate phenotype study confirming 23 possible gene-early adversity interaction in psychosis outcome: A general population twin study

Chapter 3 Subjective experience of cognitive failures as possible risk factor for negative symptoms of psychosis in the general population

Chapter $4 \quad$ Semantic activation in patients with a psychotic disorder and first-degree relatives

Chapter 5 Electrophysiological correlates of automatic spreading of activation in patients with psychotic disorder and first-degree relatives

$\begin{array}{llr}\text { Chapter } 6 & \text { Epilogue } & 121 \\ & \text { Summary } & 139 \\ \text { Samenvatting } & 145 \\ & \\ & \text { Dankwoord } & 151 \\ \text { Curriculum Vitae } & 155 \\ \text { List of publications } & 157\end{array}$
121 139 145 151 155 

CHAPTER 1

Introduction 


\section{Phenomenology of psychosis}

Psychotic disorder refers to a mental state that is characterised by a severe distortion in recognising and comprehending reality. Of all psychotic disorders, schizophrenia is the most severe mental health illness, with an estimated lifetime prevalence of approximately $1 \%$ in the population (Jablensky, et al., 1992). The core symptoms of psychotic disorders include delusions, hallucinations, disorganized speech, chaotic and catatonic behaviour, and negative symptoms (e.g., flat affect, poverty of speech, and avolition) (APA, 1994). Positive symptoms, such as hallucinations or delusions, are experiences that are not present in healthy individuals and are more transient in nature, whereas negative symptoms tend to be chronic and reflect an absence of certain abilities and impulses present in healthy individuals (Green, 2001).

However, the positive and negative classification may not be best account for the heterogeneity of the psychosis phenotype. Affective symptoms also frequently appear in patients with psychotic disorder and can be seen as an inherent part of the psychosis phenotype (Krabbendam, et al., 2004; Stefanis, et al., 2002). Positive symptoms can further be subdivided into reality distortion and disorganization symptoms (e.g., disorganized speech) (Peralta, et al., 1992), and cognitive deficits form an essential component of psychotic disorders (Dollfus, et al., 2002; Heinrichs \& Zakzanis, 1998). These cognitive impairments are central aspects of psychotic disorders, although they are not currently part of the classification criteria in the DSM-IV.

Although a syndrome-oriented categorical representation of psychosis, which makes a distinction between either the presence or absence of the disorder, is still employed in clinical practice, it does not accurately reflect the psychosis phenotype in nature. Considering the heterogeneous manifestation of the disorder, the psychosis phenotype may be better represented by a distribution along a continuum, extending from subclinical symptoms in the healthy population to clinical disorder, rather than an all-or-none phenomenon (Johns \& van Os, 2001; Linscott \& van Os, 2010; van Os, et al., 2000; van Os $\&$ Kapur, 2009). These subclinical psychotic experiences, commonly referred to as schizotypy, psychosis proneness or at risk mental states, share many of the epidemiological and phenomenological characteristics of the clinical disorder, supporting the notion of continuity between the clinical and the subclinical phenotype (van Os, et al., 1999). Investigating psychotic symptoms in non-clinical populations with a higher than 
average risk of developing the disorder, for example, psychometrically identified individuals with high schizotypy scores or first-degree relatives of patients with psychotic disorder may further elucidate the underlying aetiology of psychosis. The strategy of studying at risk populations has the advantage that potential confounding factors that are inherent to clinical patients, e.g., antipsychotic drug treatment, attention capacity or a chronic state of psychotic disorder, can be avoided.

\section{Aetiology of psychosis}

Psychotic disorder is a very heterogeneous, complex disorder with multiple causes. Genes and environmental factors are likely to interact in its aetiology. The heritability of psychosis has been investigated in family studies. First-degree relatives of patients with schizophrenia have an approximately ten times higher risk of becoming ill than individuals from the general population (Kendler \& Diehl, 1993). In twin studies, it was shown that dizygotic twins, who share about $50 \%$ of their genes, have a concordance rate of about $10 \%$ for developing the disorder, whereas for monozygotic twins, who share $100 \%$ of their genes, this rate was up to $50 \%$ (Tsuang, et al., 2001). This finding indicates that genetic factors alone are not a sufficient cause and that environmental factors also play an important role. Environmental factors that have been found to increase the risk of developing psychosis are for example: childhood abuse (Read, et al., 2005), urbanicity (van Os, 2004), stress in daily life (Myin-Germeys, et al., 2005) or minority status (van Os, et al., 2005).

Thus, the high heritability of psychosis results not only from genetic factors but also in interaction with environmental influences. While the identification of the molecular genetic variation associated with psychosis is still in an exploratory phase (van Os \& Murray, 2008), it was found that genetic factors affect some measurable heritable traits that underlie psychosis, which are closer to alterations in gene function than the diagnostic category of the psychosis phenotype (van Os \& Kapur, 2009). Thus, research has turned to the intermediate phenotype strategy, which is not only useful in understanding the genetic basis of psychosis (Braff, et al., 2007) but is also useful in studies investigating gene-environment interactions. 


\section{Vulnerability and intermediate phenotypes}

The search for causal mechanisms of psychosis has led to increased interest in the study of intermediate phenotypes. Intermediate phenotypes, also called endophenotypes, are measurable and trait-related characteristics that are thought to be closely linked to heritable risk factors. They reflect the genetic predispositions of an individual to the disorder, without the expression of the diagnostically determined phenotype, thereby providing a more reliable index of liability than the illness itself (Braff, et al., 2007; Gottesman \& Gould, 2003). Gottesman \& Gould (2003) defined five criteria of an intermediate phenotype: association with the illness in the population; heritability; state independence; cosegregation with the illness within families; and present in unaffected relatives to a higher degree than in the general population. These biologically based risk markers have a less complex genetic basis than the heterogeneous psychosis phenotype and thus, reduce the complexity of genetic research (Cannon, 2005; van Os \& Kapur, 2009). Intermediate phenotypes of psychopathology can be found in neuroanatomy, neurophysiology, neurodevelopment, biochemistry, endocrinology (Braff, et al., 2007) and in several areas of cognition (Faraone, et al., 2000; Guerra, et al., 2009; Keefe, et al., 2006b; Pflueger, et al., 2007; Sitskoorn, et al., 2004).

\section{Cognitive intermediate phenotypes}

Cognitive impairments are core features of psychotic disorders and affect a range of functions that appear to be moderately to severely impaired in most patients with the disease (Dickinson, et al., 2007; Fioravanti, et al., 2005; Heinrichs \& Zakzanis, 1998). They are not just the result of the profound impact of the symptoms on cognitive functioning in patients or the current treatments of the illness (Green, et al., 2004), as deficits remain relatively stable during the course of the illness (Ojeda, et al., 2007; Saykin, et al., 1994). Cognitive impairments are independent of positive symptoms and are particularly associated with the negative symptom domain (Dominguez, et al., 2009; Keefe, et al., 2006a). Impairments are found across various domains of cognitive functioning, including impairments of general intellectual functioning, attention, executive functioning, language, and memory (Heinrichs \& Zakzanis, 1998; Keefe, et al., 2006a). 
There is evidence that first-degree relatives of patients with psychotic disorder and people with psychometrically defined risk for the disorder also have cognitive alterations in similar domains, yet to a lesser degree than patients (Krabbendam, et al., 2001; Kremen, et al., 1994; Sitskoorn, et al., 2004; Voglmaier, et al., 1997). The findings that cognitive deficits are (i) heritable, (ii) present in relatives of patients with psychotic disorder and in individuals from the general population with subclinical psychotic symptoms, (iii) cluster in families of patients with psychotic disorder, and (iv) are associated with psychotic disorder and not a consequence of the illness, support the suggestion that deficits in cognitive functioning are a promising indicator of psychosis liability and can be seen as useful intermediate phenotype of psychosis (Gottesman \& Gould, 2003).

Thus, cognitive impairment is a central aspect of psychotic disorder, and it is well recognised that deficits in cognitive functioning can be useful intermediate phenotypes in studying psychosis. However, it is less clear how cognitive intermediate phenotypes are associated with the mechanisms of symptom formation in psychosis and how they interact with environmental influences. Therefore, the focus of this thesis is to examine whether the presence of various cognitive intermediate phenotypes is associated with specific symptoms of psychosis and how environmental risk factors interact with cognitive intermediate phenotypes in the development of specific symptoms of psychosis.

Various approaches of cognition were examined. Cognitive speed (Chapter 2) and subjective cognitive failures (Chapter 3 ), as more broadly or general defined cognition, and the semantic network system (Chapters 4 and 5), as more specifically defined cognition, were investigated to increase the understanding of the role of intermediate phenotypes of psychosis in relation to the aetiology of symptoms and the interaction with the environment.

\section{Cognitive speed}

Information processing speed, which refers to the speed at which different cognitive operations can be executed, is a central feature of the cognitive impairment seen in patients with psychotic disorder. Cognitive speed represents a general process underlying cognitive functioning, as many cognitive operations (e.g., decision making, encoding, and retrieval operations) depend on speed to a considerable degree (Dickinson, et al., 2007; Salthouse, 1996). Several previous studies indicated that cognitive speed, as an objectively measurable cognitive disturbance, is a promising intermediate phenotypic marker of 
psychosis liability, as deficits were found in relatives of patients with psychosis and in individuals with a psychometrically defined risk for the disorder (Dickinson, et al., 2007; Jabben, et al., 2007; Krabbendam, et al., 2001; Simons, et al., 2007). Accordingly, the use of cognitive speed as a liability marker of psychosis seems useful as an indirect measure of genetic risk. However, genetic mechanisms related to psychotic disorder are likely to interact with environmental factors in the aetiology of psychosis (van Os, et al., 2008). The study presented in chapter 2 applies the intermediate phenotype approach to the study of gene-environment interactions in the formation of subclinical psychosis, using cognitive speed as an indirect measure of genetic risk and childhood trauma as an environmental risk factor. A cross-twin, cross-trait design was used to investigate the gene-environment interaction between genetic risk (measured with cognitive speed) and childhood trauma in the formation of psychotic symptoms in a general population sample. This method has been validated in previous studies by our group (Lataster, et al., 2009; Wichers, et al., 2008; Wichers, et al., 2007), and the design typically examines the association between one trait measured in the proband twin and another trait measured in the co-twin. Given that one trait can be contaminated with the other trait in the same person, this method offers the possibility of examining the relationship between two traits in an unconfounded fashion.

\section{Subjective experience of cognitive failures}

Although objectively measurable cognitive disturbances such as cognitive speed have been found to be promising intermediate phenotypes, there is less evidence whether subjective experiences of cognitive functioning can also be seen as an indicator of genetic risk. The Cognitive Failure Questionnaire (CFQ) is a questionnaire that measures the selfreported frequency of subjective cognitive failures in daily life. Subjective cognitive failures are cognitively based mistakes on simple tasks that can normally be completed without error, such as throwing out a new pen and keeping the old one or failing to notice something relevant (Broadbent, et al., 1982; Wallace, et al., 2002). The experience of cognitive failures was found to be related to anxiety and depression (Merckelbach, et al., 1996; Power, 1988), and it has been suggested that abnormalities of cognitive mechanisms that are relevant to anxiety and depression may also play a role in the formation of psychotic symptoms (Garety, et al., 2001; Taylor \& John, 2004). Examining the relationship between subjective cognitive failures and psychotic symptoms may, 
therefore, further our understanding of the underlying mechanisms of psychotic symptom formation.

The study presented in chapter 3 examines the relationship between subjective cognitive failures and the formation of subclinical psychotic symptoms in a longitudinal general population family study. A longitudinal general population design makes it possible to investigate the de novo emergence of psychotic symptoms with the advantage of avoiding any confounding influence of patient status. A cross-trait, within and between-relative approach was used to test a) whether there is an association of both traits within individuals and b) whether there is familial continuity between both traits by testing the association between one trait measured in one family member and another trait measured in the other family member.

\section{Semantic network system as a specific neurocognitive intermediate phenotype}

Abnormalities in language are among the most prominent psychopathological features of psychotic disorders. Many patients exhibit an inadequate use of semantic and relational aspects of language, which are experienced as disorganized speech by the listener (Goldberg, et al., 1998). Disorganized speech is characterised by loose associations, derailments or flight of ideas (ideas slip off the track from one topic to another), irrelevant or tangential responses (answers questions in an irrelevant manner), circumstantialities or loss of goal (indirect and delayed pattern of speech), and intrinsic illogicality (Andreasen, 1979). It has been assumed that disorganized speech objectively reflects the underlying conceptual disorganization or 'thought disorder' (Aloia, et al., 1998; Spitzer, 1997). When first described by Bleuler (Bleuler, 1950), 'disturbances of associations' were considered not only as a clinical phenomenon but also as a possibility to understand and identify the neurocognitive mechanisms and dysfunctions involved in psychotic disorder (Salisbury, 2008). To shed light on processes presumably underlying disorganized speech in patients with psychotic disorder, more recent theories propose that disorganization symptoms result from abnormalities in how concepts activate one another in semantic memory (Nestor, et al., 1998; Spitzer, 1997).

The semantic priming technique is one of the most commonly used techniques to assess the semantic architecture of associations in patients with psychotic disorder. Semantic priming refers to the facilitation of responding to a target stimulus more quickly if the target is preceded by a semantically related stimulus (prime) compared to an unrelated 
stimulus (Collins \& Loftus, 1975). This facilitation is proposed to occur as semantic knowledge is organised in networks, and the related words benefit from facilitation processes in the semantic network through an automatic spreading of activation from the activated prime (Collins \& Loftus, 1975). According to this model, disorganized speech in patients with psychotic disorder results from an abnormal increase of spreading activation in the semantic memory network (Spitzer, 1997).

In the search for underlying risk factors and biological markers for psychosis, behavioural measures of neurocognitive functioning and neurophysiological assessments of brain systems are promising potential intermediate phenotypes. Alterations in the semantic network were found in patients with psychotic disorder and, to a lesser extent than in patients, in individuals at risk for psychosis. Abnormal priming effects were detected at the behavioural level (Kerns \& Berenbaum, 2000; Moritz, et al., 1999; Moritz, et al., 2001; Spitzer, 1997) and at the neurophysiological level (Kreher, et al., 2008; Mathalon, et al., 2010; Niznikiewicz, et al., 2004), which is consistent with broader or increased spreading activation.

The influence of semantic network mechanisms on symptom formation in psychosis was investigated at the behavioural (Chapter 4) and at the neurophysiological level (Chapter 5) in patients with psychotic disorder as well as their first-degree relatives. 


\section{Aims and outline of this thesis}

The overall aim of this thesis was to investigate whether the presence of various cognitive intermediate phenotypes is associated with specific symptoms of psychosis and how environmental risk factors interact with cognitive intermediate phenotypes in the development of specific symptoms of psychosis. In addition to patients with a psychotic disorder, the inclusion of research groups with a higher than average psychometric or familial risk for developing psychosis offers the possibility to investigate hypotheses not only related to alterations in cognitive mechanisms in patients with a lifetime history of a nonaffective psychotic disorder but also related to whether alterations in cognitive mechanisms demonstrate a liability for psychosis.

The first aim of this thesis was to investigate more broadly or generally defined cognitive functions as potential cognitive intermediate phenotypes in the general population. In chapter 2 we investigated whether cognitive speed serves as an intermediate phenotype of psychosis liability and whether cognitive speed interacts with childhood trauma in the formation of positive psychotic symptoms in a general population twin sample. In chapter 3 we examined in a population-based study whether proneness to subjective cognitive failures increases the risk for the development of psychotic symptoms and to what degree any association was familial.

The second aim of this thesis was to investigate whether a more specifically defined cognitive mechanism, namely the semantic network system, is abnormal in patients with psychotic disorder, and whether the semantic network system can be considered a cognitive intermediate phenotype for psychosis. In chapter 3, the picture-word interference task, a behavioural task exploring semantic processes in speech production, was used to investigate the categorical interference and associative/indirect facilitation pathways of the semantic network in patients with a psychotic disorder and healthy controls. These effects were also examined in first-degree relatives of patients with psychotic disorder, and we investigated whether an abnormal semantic network system could serve as an intermediate phenotype of psychosis liability. In chapter 4, the physiological correlates of semantic priming were examined using event-related potentials in patients with a psychotic disorder and healthy controls. Again, we assessed whether 
first-degree relatives of patients with psychotic disorder showed an aberrant semantic network system at the neurophysiological level.

Finally, a concise resume and integrative overview of the presented studies will be given in chapter 6, together with a discussion of the implications for clinical practice as well as directions for future research. 


\section{References}

Aloia, M. S., Gourovitch, M. L., Missar, D., Pickar, D., Weinberger, D. R. \& Goldberg, T. E. (1998). Cognitive substrates of thought disorder, II: specifying a candidate cognitive mechanism. Am J Psychiatry, 155, 1677-1684.

Andreasen, N. C. (1979). Thought, language, and communication disorders. I. Clinical assessment, definition of terms, and evaluation of their reliability. Arch Gen Psychiatry, 36(12), 1315-1321.

APA (1994). DSM-IV: Diagnostic and Statistical Manual of Mental Disorders (4 ed.). Washington,DC: The Association.

Bleuler, E. (1950). Dementia praecox or the group of schizophrenias. [Translated by J. Zinkin]. New York: International Universities Press.

Braff, D. L., Freedman, R., Schork, N. J. \& Gottesman, II (2007). Deconstructing schizophrenia: an overview of the use of endophenotypes in order to understand a complex disorder. Schizophr Bull, 33(1), 21-32.

Broadbent, D. E., Cooper, P. F., FitzGerald, P. \& Parkes, K. R. (1982). The Cognitive Failures Questionnaire (CFQ) and its correlates. Br J Clin Psychol, 21(1), 1-16.

Cannon, T. D. (2005). The inheritance of intermediate phenotypes for schizophrenia. Curr Opin Psychiatry, 18(2), $135-140$

Collins, A. M. \& Loftus, E. F. (1975). A spreading activation theory of semantic processing. Psychol Rev, 82, 407428.

Dickinson, D., Ramsey, M. E. \& Gold, J. M. (2007). Overlooking the obvious: a meta-analytic comparison of digit symbol coding tasks and other cognitive measures in schizophrenia. Arch Gen Psychiatry, 64(5), 532542.

Dollfus, S., Lombardo, C., Benali, K., Halbecq, I., Abadie, P., Marie, R. M., et al. (2002). Executive/attentional cognitive functions in schizophrenic patients and their parents: a preliminary study. Schizophr Res, 53(1-2), 93-99.

Dominguez, M. G., Viechtbauer, W., Simons, C. J., van Os, J. \& Krabbendam, L. (2009). Are psychotic psychopathology and neurocognition orthogonal? A systematic review of their associations. Psychol Bull, 135(1), 157-171.

Faraone, S. V., Seidman, L. J., Kremen, W. S., Toomey, R., Pepple, J. R. \& Tsuang, M. T. (2000). Neuropsychologic functioning among the nonpsychotic relatives of schizophrenic patients: the effect of genetic loading. Biol Psychiatry, 48(2), 120-126.

Fioravanti, M., Carlone, O., Vitale, B., Cinti, M. E. \& Clare, L. (2005). A meta-analysis of cognitive deficits in adults with a diagnosis of schizophrenia. Neuropsychol Rev, 15(2), 73-95

Garety, P. A., Kuipers, E., Fowler, D., Freeman, D. \& Bebbington, P. E. (2001). A cognitive model of the positive symptoms of psychosis. Psychol Med, 31(2), 189-195.

Goldberg, T. E., Aloia, M., Gourovitch, M., Missar, D., Pickar, D. \& Weinberger, D. (1998). Cognitive Substrates of Thought Disorder, I: The Semantic System. Am J Psychiatry, 155, 1671-1676.

Gottesman, II \& Gould, T. D. (2003). The endophenotype concept in psychiatry: etymology and strategic intentions. Am J Psychiatry, 160(4), 636-645.

Green, M. F. (2001). Schizophrenia Revealed - From neurons to social interactions. New York: W.W. Norton \& Company Inc.

Green, M. F., Nuechterlein, K. H., Gold, J. M., Barch, D. M., Cohen, J., Essock, S., et al. (2004). Approaching a consensus cognitive battery for clinical trials in schizophrenia: the NIMH-MATRICS conference to select cognitive domains and test criteria. Biol Psychiatry, 56(5), 301-307.

Guerra, S., Ibáñez, A., Martín, M., Bobes, M. A., Reyes, A., Mendoza, R., et al. (2009). N400 deficits from semantic matching of pictures in probands and first-degree relatives from multiplex schizophrenia families. Brain Cogn, 70(2), 221-230.

Heinrichs, R. W. \& Zakzanis, K. K. (1998). Neurocognitive deficit in schizophrenia: a quantitative review of the evidence. Neuropsychology, 12(3), 426-445.

Jabben, N., van Os, J., Janssen, I., Versmissen, D. \& Krabbendam, L. (2007). Cognitive alterations in groups at risk for psychosis: neutral markers of genetic risk or indicators of social disability? Acta Psychiatr Scand, 116(4), 253-262.

Jablensky, A., Sartorius, N., Ernberg, G., Anker, M., Korten, A., Cooper, J. E., et al. (1992). Schizophrenia: manifestations, incidence and course in different cultures. A World Health Organization ten-country study. Psychol Med Monogr Suppl, 20, 1-97.

Johns, L. C. \& van Os, J. (2001). The continuity of psychotic experiences in the general population. Clin Psychol Rev, 21(8), 1125-1141. 
Keefe, R. S., Bilder, R. M., Harvey, P. D., Davis, S. M., Palmer, B. W., Gold, J. M., et al. (2006a). Baseline neurocognitive deficits in the CATIE schizophrenia trial. Neuropsychopharmacol, 31(9), 2033-2046.

Keefe, R. S., Perkins, D. O., Gu, H., Zipursky, R. B., Christensen, B. K. \& Lieberman, J. A. (2006b). A longitudinal study of neurocognitive function in individuals at-risk for psychosis. Schizophr Res, 88(1-3), 26-35.

Kendler, K. S. \& Diehl, S. R. (1993). The genetics of schizophrenia: a current, genetic-epidemiologic perspective. Schizophr Bull, 19(2), 261-285.

Kerns, J. G. \& Berenbaum, H. (2000). Aberrant semantic and affective processing in people at risk for psychosis. J Abnorm Psychol, 109(4), 728-732.

Krabbendam, L., Marcelis, M., Delespaul, P., Jolles, J. \& van Os, J. (2001). Single or multiple familial cognitive risk factors in schizophrenia? Am J Med Genet, 105(2), 183-188.

Krabbendam, L., Myin-Germeys, I., De Graaf, R., Vollebergh, W., Nolen, W. A., ledema, J., et al. (2004). Dimensions of depression, mania and psychosis in the general population. Psychol Med, 34(7), 11771186.

Kreher, D. A., Holcomb, P. J., Goff, D. \& Kuperberg, G. R. (2008). Neural Evidence for Faster and Further Automatic Spreading Activation in Schizophrenic Thought Disorder. Schizophr Bull, 34(3), 473-482.

Kremen, W. S., Seidman, L. J., Pepple, J. R., Lyons, M. J., Tsuang, M. T. \& Faraone, S. V. (1994). Neuropsychological risk indicators for schizophrenia: a review of family studies. Schizophr Bull, 20(1), 103-119.

Lataster, T., Wichers, M., Jacobs, N., Mengelers, R., Derom, C., Thiery, E., et al. (2009). Does reactivity to stress cosegregate with subclinical psychosis? A general population twin study. Acta Psychiatr Scand, 119(1), 45-53.

Linscott, R. J. \& van Os, J. (2010). Systematic Reviews of Categorical Versus Continuum Models in Psychosis: Evidence for Discontinuous Subpopulations Underlying a Psychometric Continuum. Implications for DSM-V, DSM-VI, and DSM-VII. Annu Rev Clin Psychol, 6(1), 391-419.

Mathalon, D. H., Roach, B. J. \& Ford, J. M. (2010). Automatic semantic priming abnormalities in schizophrenia. Int J Psychophysiol, 75(2), 157-166.

Merckelbach, H., Muris, P., Nijman, H. \& de Jong, P. J. (1996). Self-reported cognitive failures and neurotic symptomatoloy. Pers Indiv Differ, 20(6), 715-724.

Moritz, S., Andresen, B., Domin, F., Martin, T., Probsthein, E., Kretschmer, G., et al. (1999). Increased automatic spreading activation in healthy subjects with elevated scores in a scale assessing schizophrenic language disturbances. Psychol Med, 29(1), 161-170.

Moritz, S., Mersmann, K., Kloss, M., Jacobsen, D., Wilke, U., Andresen, B., et al. (2001). 'Hyper-priming' in thought-disordered schizophrenic patients. Psychol Med, 31(2), 221-229.

Myin-Germeys, I., Delespaul, P. \& van Os, J. (2005). Behavioural sensitization to daily life stress in psychosis. Psychol Med, 35(5), 733-741.

Nestor, P. G., Shenton, M. E., Wible, C., Hokama, H., O'Donnell, B. F., Law, S., et al. (1998). A neuropsychological analysis of schizophrenic thought disorder. Schizophr Res, 29(3), 217-225.

Niznikiewicz, M. A., Friedman, M., Shenton, M. E., Voglmaier, M., Nestor, P. G., Frumin, M., et al. (2004). Processing sentence context in women with schizotypal personality disorder: An ERP study. Psychophysiology, 41(3), 367-371.

Ojeda, N., Sanchez, P., Elizagarate, E., Yoller, A. B., Ezcurra, J., Ramirez, I., et al. (2007). Course of cognitive symptoms in schizophrenia: a review of the literature. Actas Esp Psiquiatr, 35(4), 263-270.

Peralta, V., de Leon, J. \& Cuesta, M. J. (1992). Are there more than two syndromes in schizophrenia? A critique of the positive-negative dichotomy. Br J Psychiatry, 161, 335-343.

Pflueger, M. O., Gschwandtner, U., Stieglitz, R. D. \& Riecher-Rossler, A. (2007). Neuropsychological deficits in individuals with an at risk mental state for psychosis - working memory as a potential trait marker. Schizophr Res, 97(1-3), 14-24.

Power, M. J. (1988). Cognitive failures, dysfunctional attitudes, and symptomatology: A longitudinal study. Cog.Emo, 2(2), 133-143.

Read, J., van Os, J., Morrison, A. P. \& Ross, C. A. (2005). Childhood trauma, psychosis and schizophrenia: a literature review with theoretical and clinical implications. Acta Psychiatr Scand, 112(5), 330-350.

Salisbury, D. F. (2008). Semantic activation and verbal working memory maintenance in schizophrenic thought disorder: insights from electrophysiology and lexical ambiguity. Clin EEG Neurosci, 39(2), 103-107.

Salthouse, T. A. (1996). The processing-speed theory of adult age differences in cognition. Psychol Rev, 103(3), 403-428.

Saykin, A. J., Shtasel, D. L., Gur, R. E., Kester, D. B., Mozley, L. H., Stafiniak, P., et al. (1994). Neuropsychological deficits in neuroleptic naive patients with first-episode schizophrenia. Arch Gen Psychiatry, 51(2), 124131. 
Simons, C. J., Jacobs, N., Jolles, J., van Os, J. \& Krabbendam, L. (2007). Subclinical psychotic experiences and cognitive functioning as a bivariate phenotype for genetic studies in the general population. Schizophr Res, 92(1-3), 24-31.

Sitskoorn, M. M., Aleman, A., Ebisch, S. J., Appels, M. C. \& Kahn, R. S. (2004). Cognitive deficits in relatives of patients with schizophrenia: a meta-analysis. Schizophr Res, 71(2-3), 285-295.

Spitzer, M. (1997). A cognitive neuroscience view of schizophrenic thought disorder. Schizophr Bull, 23(1), 29-50.

Stefanis, N. C., Hanssen, M., Smirnis, N. K., Avramopoulos, D. A., Evdokimidis, I. K., Stefanis, C. N., et al. (2002). Evidence that three dimensions of psychosis have a distribution in the general population. Psychol Med, 32(2), 347-358.

Taylor, J. L. \& John, C. H. (2004). Attentional and memory bias in persecutory delusions and depression. Psychopathology, 37(5), 233-241.

Tsuang, M. T., Stone, W. S. \& Faraone, S. V. (2001). Genes, environment and schizophrenia. Br J Psychiatry, $178(40), 18-24$

van Os, J. (2004). Does the urban environment cause psychosis? Br J Psychiatry, 184, 287-288.

van Os, J., Hanssen, M., Bijl, R. V. \& Ravelli, A. (2000). Strauss (1969) revisited: a psychosis continuum in the general population? Schizophr Res, 45(1-2), 11-20.

van Os, J. \& Kapur, S. (2009). Schizophrenia. Lancet, 374(9690), 635-645.

van Os, J., Krabbendam, L., Myin-Germeys, I. \& Delespaul, P. (2005). The schizophrenia envirome. Curr Opin Psychiatry, 18(2), 141-145.

van Os, J. \& Murray, R. (2008). Introduction. Schizophr Bull, 34(6), 1064-1065.

van Os, J., Rutten, B. P. \& Poulton, R. (2008). Gene-Environment Interactions in Schizophrenia: Review of Epidemiological Findings and Future Directions. Schizophr Bull, 34(6), 1066-1082.

van Os, J., Verdoux, H., Maurice-Tison, S., Gay, B., Liraud, F., Salamon, R., et al. (1999). Self-reported psychosislike symptoms and the continuum of psychosis. Soc Psychiatry Psychiatr Epidemiol, 34(9), 459-463.

VogImaier, M. M., Seidman, L. J., Salisbury, D. \& McCarley, R. W. (1997). Neuropsychological dysfunction in schizotypal personality disorder: a profile analysis. Biol Psychiatry, 41(5), 530-540.

Wallace, J. C., Kass, S. J. \& Stanny, C. J. (2002). The cognitive failures questionnaire revisited: dimensions and correlates. J Gen Psychol, 129(3), 238-256.

Wichers, M. C., Myin-Germeys, I., Jacobs, N., Kenis, G., Derom, C., Vlietinck, R., et al. (2008). Susceptibility to depression expressed as alterations in cortisol day curve: a cross-twin, cross-trait study. Psychosom Med, 70(3), 314-318.

Wichers, M. C., Myin-Germeys, I., Jacobs, N., Peeters, F., Kenis, G., Derom, C., et al. (2007). Genetic risk of depression and stress-induced negative affect in daily life. Br J Psychiatry, 191, 218-223. 



\title{
A cognitive intermediate phenotype study confirming possible gene-early adversity interaction in psychosis outcome: A general population twin study
}

\author{
Stefanie Pfeifer ${ }^{1}$, Lydia Krabbendam ${ }^{1,2}$, Inez Myin-Germeys ${ }^{1}$, Catherine Derom ${ }^{3}$, Marieke \\ Wichers $^{1}$, Nele Jacobs ${ }^{1,4}$, Evert W. Thiery ${ }^{5}$, Jim van $0 s^{1,6}$

\footnotetext{
${ }^{1}$ Department of Psychiatry and Neuropsychology, South Limburg Mental Health Research and Teaching Network, EURON, Maastricht University, Maastricht, The Netherlands

${ }^{2}$ Centre Brain and Learning, VU University Amsterdam, Amsterdam, The Netherlands

${ }^{3}$ Department of Human Genetics, University Hospital Gasthuisberg, Katholieke Universiteit Leuven, B-3000

Leuven, Belgium

${ }^{4}$ Faculty of Psychology, Open University of the Netherlands, Heerlen, The Netherlands

${ }^{5}$ Association for Scientific Research in Multiple Births, Ghent, Belgium

${ }^{6}$ Division of Psychological Medicine, Institute of Psychiatry, London SE5 8AF, UK
}

Psychosis: Psychological, Social and Integrative Approaches 2009; 2 (1): 1 - 11. 


\section{Abstract}

Background. The association between childhood adversity and psychosis outcomes later in life could possibly be moderated by genetic risk. The aim of the study was to investigate the interaction between childhood adversity and genetic risk in the formation of psychotic symptoms, using cognitive speed as indicator of genetic risk.

Methods. In a cross-twin, cross-trait analysis of monozygotic twins in the general population, the association between childhood adversity and psychotic symptoms was examined, using a cognitive intermediary phenotype as genetic risk marker.

Results. Psychotic symptoms in the proband twin were associated with childhood adversity and, independently, with a measure of cognitive speed in the co-twin. The association between childhood adversity and psychotic symptoms was much stronger (interaction: $\chi^{2}=8.48, p=0.004$ ) if cognitive speed was worse.

Conclusion. Higher level of genetic risk associated with psychosis may moderate the impact of childhood adversity on the risk of adult psychotic symptom formation. 


\section{Introduction}

There is evidence that self-reported childhood trauma may increase the risk of psychosis (Bebbington, et al., 2004; Janssen, et al., 2004; Morgan \& Fisher, 2007; Spauwen, et al., 2006). However, given the considerable heterogeneity in psychotic response to childhood trauma (Bendall, et al., 2008), any risk-increasing effect of childhood adversity must operate in interaction with other background vulnerability factors. These may comprise biological, psychological and social factors (Larkin \& Read, 2008) and likely include the level of pre-existing liability to psychosis (Spauwen, et al., 2006). Given evidence for genetrauma interactions in the context of other psychiatric phenotypes (Caspi, et al., 2002; Wichers, et al., 2009), it is attractive to hypothesize that one mechanism by which trauma increases the risk for psychosis similarly involves moderation by genetic factors or geneenvironment interaction (van Os, et al., 2008b).

Impaired cognitive functioning is one of the core symptoms of psychotic disorder (Dollfus, et al., 2002; Heinrichs \& Zakzanis, 1998) and is also present to a lesser extent in relatives of patients i.e., individuals at genetic risk of psychosis (Faraone, et al., 2000), which has led several authors to suggest that impaired cognition is an intermediate phenotypic marker of psychosis (Green \& Nuechterlein, 2004; Keefe, et al., 2006b). Therefore, measures of cognitive impairment may be used as an indirect measure of genetic risk in studies of gene-environment interaction (GXE).

\section{Aim of the study}

The aim of the present study was to investigate the interaction between childhood adversity (CA) and cognitive impairment as an expression of genetic risk in the formation of positive psychotic symptoms (PS) at the subclinical level of the general population (van Os, et al., 2008a).Cognitive impairment was assessed with a composite measure reflecting cognitive speed, which according to a recent meta-analysis, is a robust intermediate phenotypic marker of cognitive impairment in schizophrenia (Dickinson, et al., 2007). It was hypothesized that greater levels of cognitive impairment would result in larger effects sizes in the association between CA and PS. The study sample consisted of monozygotic (MZ) and dizygotic (DZ) twins from the general population, making it possible to, first, examine whether cognitive impairment can be seen as genetic risk marker, and second, to 
model the association between CA and PS in the MZ proband twin as a function of the (psychometrically unconfounded) cognitive proxy for genetic risk in the MZ co-twin.

\section{Methods}

\section{Sample}

The study was a part of a longitudinal, general population twin study in the context of gene-environment interactions in psychopathology (Jacobs, et al., 2006; Wichers, et al., 2007). Participants were recruited from the East Flanders Prospective Twin Survey (Derom, et al., 2002; Loos, et al., 1998) and from birth registers of Flemish municipalities in Belgium (62 pairs). All twin participants were female, and all four grandparents were of Belgian origin. For further details on the sample see Jacobs et al. (2006) and Wichers et al. (2007).

The study sample initially consisted of 621 female twin participants (575 female twins and 46 of their non-twin sisters). All 46 non-twin sisters were excluded from the current analyses. Three participants were additionally excluded due to missing information on zygosity.

\section{Instruments}

Assessment of subclinical psychotic experiences. Subclinical psychotic experiences were assessed with the Community Assessment of Psychic Experiences (CAPE) (Stefanis, et al., 2002), a self-report questionnaire rating attenuated affective and nonaffective psychotic experiences. The CAPE measures on a dimensional scale the frequency of the experiences (from 'never (1)' to 'nearly always (4)'). A weighted score was calculated for each frequency to account for partial non-response (Konings, et al., 2006; Stefanis, et al., 2002). The standardized total score was used in the analysis.

The present study focused on the outcome of the positive subclinical psychosis dimension. The negative and the depressive subclinical psychosis dimension were used as covariates in the analyses in order to assess specificity of any significant association with the positive dimension.

Assessment of childhood adversity. CA was assessed using a short version of the selfreport questionnaire based on the Dutch translation of the original 70-item Childhood 
Trauma Questionnaire (CTQ) (Arntz \& Wessel, 1996; Bernstein, et al., 1994). The short CTQ is a 28 -item-self-report inventory assessing retrospectively emotional and physical neglect and abuse, and sexual abuse. At the request of the Twin Registry, the most explicit items concerning sexual and physical abuse were omitted, resulting in 21 items. Each item is scored on a five-point Likert scale from 'never' to 'always', reflecting the degree of agreement with the statement. Cronbach's alpha for this 21-item questionnaire was 0.9347 .

Assessment of genetic risk. Speed of information processing (hereafter: cognitive speed) was used as marker of genetic risk. Based on previous principal component factor analysis of the same twin sample (for detailed description of factor analysis of the same sample see (Simons, et al., 2007)) the factor 'cognitive speed' was created from performance indices on the Stroop Colour-Word Test, Concept Shifting test (a modified version of the Trail Making Test) and the Letter Digit Substitution Test (a modified version of the Symbol Digit Modalities Test). The factor loadings of the Stroop and the CST varied from 0.49 to 0.70 and the factor loading for the LDST was -0.66 . Since cognitive speed was measured as performance time and factor loadings on this factor were positive, positive correlations with the subclinical psychosis dimensions indicate that a higher level of symptoms was accompanied by slower reaction times.

\section{Measurement points and risk set}

Participants were interviewed at five measurement points (TO-T4) over a mean period of 15 months. CA and cognitive speed were assessed at baseline (hereafter TO). The CAPE questionnaire was administered at TO, T2 and T4. All three measurement points from each subject were used in the current study.

The risk set consisted of all twins who had completed the CTQ, the measurements of cognitive speed and the CAPE at TO, T2 and T4 and included $330 \mathrm{MZ}$ twins and $210 \mathrm{DZ}$ twins. Only twin pairs of which both twins participated in the study were included in the analysis.

\section{Analysis}

Multilevel linear regression analysis, using the XTMIXED command in STATA version 10.0 (StataCorp, 2007) was applied to the data, using twin-pair and individual measurement 
occasion as the levels at which the data were hierarchically clustered. Effect sizes were expressed as $B$, the fixed regression coefficient of the predictor.

Analyses were adjusted for the a priori demographic confounders age and level of education, and additionally for the negative and the depressive dimension of the subclinical psychosis phenotype as indicated above.

Main effects and interactions were assessed using the Wald test (Clayton \& Hill, 1993). In the case of significant interaction, stratified effect sizes were calculated from linear combinations of coefficients, using the LINCOM command in STATA.

Cognitive speed as indicator of genetic risk. The first step in the analysis was to assess whether cognitive speed can be validly interpreted as a marker of genetic risk of the outcome of PS. This would require demonstrating stronger cross-twin, cross-trait correlations between PS and cognitive speed in MZ twins as compared to DZ twins. PS in the proband twin were regressed on the interaction between zygosity and cognitive speed in the co-twin. This was followed by the calculation of effect sizes of the association between PS in the proband twin and cognitive speed in the co-twin for MZ and DZ twins separately. If there is a significant main effect of cognitive speed in the co-twin on PS in the proband twin for $M Z$ twins but not for DZ twins, cognitive speed can be interpreted as a marker of genetic risk.

Interaction between cognitive speed and CA in the outcome of PS. The interaction analyses were carried out in genetically identical $M Z$ twins only to ensure that the interpretation of cognitive impairment as genetic risk marker was valid. In the main interaction analyses, the variable $z$ (cognitive speed) in the $M Z$ co-twin was included as possible moderator of the association between variable $x$ (CA) and the outcome $y$ (PS) in the proband twin. The reason for measuring the moderator variable $z$ in the co-twin was that the outcome $y$ is likely contaminated by the variable $z$ when measured in the same person (see Figure 1). In order to conduct these analyses, a data set was generated that contained all possible pairwise relationships between $\mathrm{MZ}$ twins.

First, linear regression analyses were conducted to investigate the association between CA and $\mathrm{PS}$ within $\mathrm{MZ}$ twins. 
Second, the association between $C A$ in the $M Z$ proband twin and cognitive speed in the co-twin was assessed using multilevel linear regression analysis, in order to test the required independence between the variables making up the interaction term.

Third, PS in the MZ proband twin were regressed on the interaction between CA in the proband twin and cognitive speed in the co-twin, in order to test the hypothesis that genetic risk moderates the association between CA and PS. The LINCOM procedure was applied to calculate stratified effect sizes in the group at high (worse cognitive speed) versus low levels of genetic risk.

\section{Results}

\section{Sample}

A total of $165 \mathrm{MZ}$ female twin pairs were included in the analyses. A further $110 \mathrm{DZ}$ female twin pairs were additionally included in the first analysis investigating cognitive speed as indicator of genetic risk. Mean age was 26.8 years $(S D=7.35$, range: 18 - 46 years) and 27.5 years ( $S D=7.71$, range: 18 - 46 years) for $M Z$ and $D Z$ twins, respectively. In MZ twins, a majority of $60 \%$ had a higher education, $37 \%$ followed higher secondary school and $3 \%$ finished primary education only. In the DZ twins, these figures were $63 \%, 35 \%$ and $2 \%$, respectively. The majority of $M Z$ and $D Z$ twins was currently employed ( $M Z$ twins (DZ): $61 \%(65 \%)$ employed, 34\% (30\%) student, 3\% (1\%) unemployed, $1 \%(4 \%)$ homemaker and $1 \%$ sick leave). See Table 1 for descriptives of the CAPE, the CTQ and the neuropsychological measures.

\section{Cognitive speed as genetic risk marker}

The correlation in MZ twin pairs between PS in the proband twin and cognitive speed in the co-twin were higher $(r=0.16)$ than in DZ twin pairs $(r=0.056)$, suggesting that cognitive speed provides a valid indicator of genetic risk for psychotic experiences. Correlations between cognitive speed and the PS in MZ and DZ twins are shown in Table 2.

Multilevel linear regression analyses showed a significant interaction between the variable zygosity and cognitive speed in the co-twin in the model of PS $\left(\chi^{2}=4.26, p=0.039\right)$. After adjustment for the a priori selected confounders, the association was only marginally reduced $\left(\chi^{2}=3.76, p=0.052\right)$. 
There was a significant association between cognitive speed in the co-twin and PS in the proband twin in $\mathrm{MZ}$ twins $(B=0.16,95 \% \mathrm{Cl}: 0.06,0.26, \mathrm{p}=0.002$ ), but not in $\mathrm{DZ}$ twins ( $B=$ $0.004,95 \% \mathrm{Cl}:-0.11,0.12, \mathrm{p}=0.947)$, suggesting that cognitive speed can be interpreted as a valid genetic marker of risk of psychosis.

\section{Interaction between proxy genetic risk and childhood adversity}

Linear regression analyses showed a significant main effect of CA on PS in MZ twins ( $B=$ $0.52,95 \% \mathrm{Cl}: 0.35,0.67, \mathrm{p}<0.000)$. This association remained significant after adjustment for the a priori selected confounders, the negative and depressive subclinical dimension and cognitive speed in the co-twin ( $B=0.28,95 \% \mathrm{Cl}: 0.12,0.41, \mathrm{p}<0.000)$.

Second, linear regression analysis in the $M Z$ twins showed no significant association between $\mathrm{CA}$ in the proband twin and cognitive speed (genetic risk) in the co-twin ( $B=0.13$, $95 \% \mathrm{Cl}:-0.06,0.33, \mathrm{p}=0.183$ ), indicating that the requirement that the variables making up the interaction term be independent was met.

Third, linear regression analyses showed a significant interaction between $C A$ in the $M Z$ proband twin and cognitive speed in the co-twin in the model of the PS outcome $\left(X^{2}=8.48\right.$, $p=0.004)$. Linear combinations of the interaction showed a stronger effect of $C A$ if the cotwin had high level of genetic risk (i.e., worse cognitive speed) $(B=0.71,95 \% \mathrm{Cl}: 0.49,0.93$, $\mathrm{p}<0.000)$ compared to if the co-twin had low level of genetic risk $(B=0.23,95 \% \mathrm{Cl}:-0.01$, $0.47, p=0.063)$. This effect did not change after additional adjustment for the $a$ priori confounders $\left(\chi^{2}=7.58, p=0.006\right.$; high genetic risk: $B=0.84,95 \% \mathrm{Cl}$ : $0.63,1.06, p<0.000$ versus low genetic risk: $B=0.41,95 \% \mathrm{Cl}: 0.16,0.65, p=0.001$ ), and was reduced but not nullified after further adjustment of negative and depressive subclinical experiences $\left(\chi^{2}=\right.$ $3.46, \mathrm{p}=0.063$; high genetic risk: $B=0.38,95 \% \mathrm{Cl}: 0.19,0.57, \mathrm{p}<0.000$ versus low genetic risk: $B=0.13,95 \% \mathrm{Cl}:-0.07,0.34, \mathrm{p}=0.213)$.

\section{Discussion}

In a general population MZ twin sample, the impact of CA on PS was moderated by level of intermediate phenotype expression of genetic risk for psychosis. That is, the association between CA and PS in one MZ twin increased with decreasing speed of information processing in the other twin. This supports the hypothesis that cognitive speed, as genetic 
risk factor, moderates the associations between CA and PS. The results remained equally strong and significant after controlling for possible demographical confounders and could not be ascribed to overlap with other dimensions within the CAPE psychosis phenotype. Genetic risk did not increase the likelihood of exposure to CA, ruling out confounding by a mechanism of genetic risk increasing likelihood of environmental exposure.

\section{Cognitive speed as indicator of genetic risk}

In line with previous studies, indicating that cognitive speed is a robust intermediate phenotypic marker of psychosis liability (Dickinson, et al., 2007; Jabben, et al., 2007) the present study showed that the link between cognitive speed and subclinical psychosis is much stronger in relatives who share all of their genetic material compared to relatives who share only part of their genetic material. Intermediate phenotypes, which are closely linked to heritable risk factors, may not only be useful in understanding the genetic basis of psychosis (Braff, et al., 2007) but also as broadly defined proxy measures of genetic risk of psychosis in studies investigating gene-environment interaction. This may represent a productive strategy whilst the identification of the molecular genetic variation associated with psychosis is still in an exploratory phase (van Os \& Murray, 2008). Using a similar approach, Weiser et al. (2007) reported that poor premorbid social and cognitive abilities, as expression of genetic risk, increased the association between urbanicity and hospitalization for schizophrenia in men (Weiser, et al., 2007).

In general, cognitive impairment seems to be more strongly correlated with negative rather than positive psychotic symptoms, both in patients with psychotic disorder (Dominguez, et al., 2009) and in first-degree family members (Grove, et al., 1991). This, however, is not necessarily discordant with our results because the attenuated expression of the different psychotic symptom dimensions of psychosis in the general population leads to more overlap between the dimensions (Stefanis, et al., 2002). It is therefore more difficult to sensitively measure and distinguish between the dimensions of the psychosis phenotype at the level of the general population compared to the clinical disorder. More importantly, it has been suggested that at the subclinical level, positive experiences are a reliable indicator of psychosis vulnerability, whereas after transition to psychotic illness, negative symptoms appear to reflect psychosis risk more reliably (Jabben, et al., 2007). This is in line with findings showing associations between cognition and positive symptoms at the subclinical level (Jabben, et al., 2007; Krabbendam, et al., 2005) and between 
cognition and negative symptoms at the clinical level (Jabben, et al., 2007; Keefe, et al., 2006a).

\section{Gene-adversity interaction in the development of positive psychotic experiences}

The finding of gene-adversity interaction is in accordance with previous studies indicating that the risk-increasing effect of CA is stronger in individuals with a familial or individual liability to psychosis (Janssen, et al., 2004; Spauwen, et al., 2006). The use of cognitive speed as one possible expression of psychosis risk may facilitate the search for the specific genetic variation associated with this interaction, as has been reported for other psychiatric phenotypes (Caspi, et al., 2002), and yields further insight into the possible mechanism of the gene-adversity interaction in psychosis.

At the neurobiological level, persistent exposure to adverse life events could modulate neurodevelopmental processes resulting in permanent changes of the underlying brain structure (Nemeroff, 2004). Specifically, it has been shown that long lasting exposure to stressors may lead to chronic glucocorticoid release, resulting in a permanent dysregulation of the hypothalamic-pituitary-adrenal (HPA) axis and structural abnormalities in the hippocampus (Teicher, et al., 2003). These HPA axis dysregulations may contribute to the dopaminergic abnormalities that are generally thought to be involved in psychosis (Read, et al., 2001; Walker \& Diforio, 1997). On the other hand, genetic liability to psychosis results in neurodevelopmental brain abnormalities, e.g., enlarged ventricles, smaller temporal lobe and smaller hippocampus (Lawrie, et al., 2008). Therefore, the increased association between CA and PS in individuals at increased genetic risk may be interpreted as the detrimental effects of CA on an already compromised developing brain. Although this effect could potentially also be expressed in cognitive impairment, suggesting an alternative explanation for the current findings, the fact that we assessed cognitive speed in the co-twin, and thus independent of any exposure to CA, renders this explanation unlikely.

At the psychological level, exposure to CA may form dysfunctional metacognitive skills which hamper coping with anomalous psychotic-like experiences, increasing the risk for true psychotic symptom formation (Bak, et al., 2005). The presence of gene-environment interaction suggests that this psychological effect of adversity is particularly harmful in those whose cognitive capacities are already affected as part of the genetic liability to psychosis. 


\section{Limitations}

The findings of the present study must be interpreted in the context of several methodological issues. First, it cannot be excluded that pre-existing psychotic experiences increased the risk for exposure to CA (i.e., person-environment correlation (van Os \& Sham, 2003)). However, this does not affect the interpretation of the current findings, which suggest that genetic risk increases the association between adversity and trauma, either by increasing the risk for persistence of prior psychotic experiences with poor prognosis, or by increasing the risk for newly developing psychotic experiences.

Second, the CTQ is a retrospective and relatively crude assessment of CA, which is based on self-report and thus verification of the traumatic event is not possible. However, in a truly prospective assessment of the effects of CA, as was done in the only population study published so far yielding negative results (Spataro, et al., 2004), findings will be biased by the fact that interventions will take place in registered cases, possibly diminishing the risk for later psychotic symptoms or general psychopathology. In the present study, CA was assessed in both twins independently and it was found that the adverse events were shared in $78 \%$ of the $M Z$ twins. However, severity and frequency of the adverse event was not assessed, and the most explicit items concerning sexual and physical abuse were omitted. Thus, differential effects of more severe types of trauma could not be assessed. For future research, it would be necessary to include more detailed assessment of childhood trauma.

Third, the interpretation of the results depends directly on the validity of the interpretation of cognitive speed as putative intermediate phenotype of psychosis. The validity was confirmed in the current study by a stronger correlation between cognitive speed in the co-twin and PS in the proband twin in MZ compared to DZ twins. However, to further confirm this interpretation, additional research may be instructive.

Forth, the findings are based on self-report. This may result in less reliable data, especially in the case of psychotic symptoms. Therefore, replication with interview data is necessary. Finally, replication is needed in a clinically diagnosed sample to extrapolate the findings to patients with the full-blown psychotic disorder. 


\section{Acknowledgments}

This work was supported by the Dutch Foundation for Scientific Research. The East Flanders Prospective Survey has been in part supported by grants from the Fund of Scientific Research, Flanders; and Twins, the Association for Scientific Research in Multiple Births, Belgium. Dr M. C. Wichers was supported by the Dutch Medical Council (VENI Grant no. 916-76-147). Dr L. Krabbendam was supported by the Netherlands Organization for Scientific Research (VIDI Grant no. 452-07-007). We thank all the participating twins for their cooperation. 
Table 1. Sample summary.

\begin{tabular}{|c|c|c|c|c|}
\hline & \multicolumn{2}{|c|}{$\mathrm{MZ}$ twins ( $\mathrm{N}=165$ pairs) } & \multicolumn{2}{|c|}{ DZ twins ( $\mathrm{N}=110$ pairs) } \\
\hline & Means (SD) & Range & Means (SD) & Range \\
\hline \multicolumn{5}{|l|}{ CAPE $^{a}$} \\
\hline Positive dimension & $1.20(0.17)$ & $1.0-2.5$ & $1.23(0.19)$ & $1.0-2.1$ \\
\hline Negative dimension & $1.53(0.33)$ & $1.0-2.9$ & $1.54(0.34)$ & $1.0-2.6$ \\
\hline Depressive dimension & $1.69(0.37)$ & $1.0-3.6$ & $1.74(0.36)$ & $1.0-3.0$ \\
\hline $\mathrm{CTQ}^{\mathrm{b}}$ & $1.67(0.58)$ & $1.0-4.2$ & $1.67(0.59)$ & $1.0-4.5$ \\
\hline \multicolumn{5}{|l|}{ Neuropsychological tasks ${ }^{c}$ : } \\
\hline Stroop names & $13.84(2.05)$ & $10.1-26.3$ & $14.31(4.69)$ & $9.3-75.4$ \\
\hline Stroop colours & $19.27(2.92)$ & $13.0-31.3$ & $20.07(4.03)$ & $13.2-40.3$ \\
\hline Stroop interference task & $32.01(6.48)$ & $18.4-58.4$ & $33.56(7.23)$ & $19.5-85.6$ \\
\hline CST numbers & $16.06(3.49)$ & $8.5-28.7$ & $16.27(3.52)$ & $10.2-28.3$ \\
\hline CST letters & $19.46(4.46)$ & $11.3-46.1$ & $19.56(6.09)$ & $12.1-75.2$ \\
\hline CST number/letter shifting & $25.30(7.78)$ & $10.9-79.2$ & $25.07(7.73)$ & $13.3-73.8$ \\
\hline LDST & $62.16(8.24)$ & $43.0-85.0$ & $60.56(7.72)$ & $40.0-83.0$ \\
\hline
\end{tabular}

Notes: CAPE, Community Assessment of Psychic Experiences; CTQ, Childhood Trauma Questionnaire; CST, Concept Shifting Test; LDST, Letter Digit Substitution Test.

${ }^{a}$ CAPE scores are weighted means of the positive, negative, and depressive dimension.

${ }^{\mathrm{b}} \mathrm{CTQ}$ scores are mean scores on trauma childhood adversity.

${ }^{c}$ Mean scores on the neuropsychological tasks are listed. 
Table 2. Correlations between the positive subclinical psychotic experiences (PS) and cognitive speed in $M Z$ and DZ twin pairs ${ }^{a}$.

\begin{tabular}{lllll}
\hline & Proband twin PS & Proband twin speed & Co-twin PS & Co-twin speed \\
\hline Proband twin PS & -------- & $\mathbf{0 . 1 1 3 2} *$ & $\mathbf{0 . 2 6 2 2} *$ & $\mathbf{0 . 1 5 8 3 ^ { * }}$ \\
Proband twin speed & 0.0254 (n.s) & ------ & $\mathbf{0 . 1 5 8 3 ^ { * }}$ & $\mathbf{0 . 5 8 7 5 *}$ \\
Co-twin PS & $0.2882^{*}$ & 0.0558 n.s. & ----- & $\mathbf{0 . 1 1 3 2 *}$ \\
Co-twin speed & 0.0558 (n.s.) & $0.4042^{*}$ & 0.0254 (n.s.) & --------- \\
\hline
\end{tabular}

${ }^{a}$ Correlations in MZ twin (165 pairs) are above the diagonal, those for DZ twin (105 pairs) are below the diagonal; n.s., non significant.

$* p<0.05$

Figure 1. Cross-twin, cross-trait design.

Interaction: The interaction term was formed by (Childhood adversity proband twin) x (Cognitive Speed co-twin) on the outcome of positive psychotic symptoms.

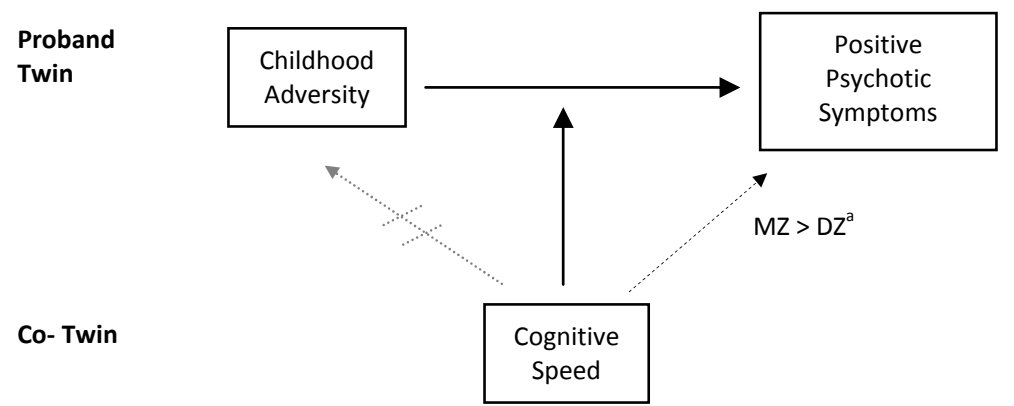

${ }^{\text {a }}$ Cognitive speed as vulnerability factor. 


\section{References}

Arntz, A. \& Wessel, I. (1996). Jeugd Trauma Vragenlijst (Dutch version of the Childhood Trauma Questionnaire). Maastricht.

Bak, M., Krabbendam, L., Janssen, I., de Graaf, R., Vollebergh, W. \& van Os, J. (2005). Early trauma may increase the risk for psychotic experiences by impacting on emotional response and perception of control. Acta Psychiatr Scand, 112(5), 360-366.

Bebbington, P. E., Bhugra, D., Brugha, T., Singleton, N., Farrell, M., Jenkins, R., et al. (2004). Psychosis, victimisation and childhood disadvantage: evidence from the second British National Survey of Psychiatric Morbidity. Br J Psychiatry, 185, 220-226.

Bendall, S., Jackson, H. J., Hulbert, C. A. \& McGorry, P. D. (2008). Childhood trauma and psychotic disorders: a systematic, critical review of the evidence. Schizophr Bull, 34(3), 568-579.

Bernstein, D. P., Fink, L., Handelsman, L., Foote, J., Lovejoy, M., Wenzel, K., et al. (1994). Initial reliability and validity of a new retrospective measure of child abuse and neglect. Am J Psychiatry, 151(8), 1132 1136.

Braff, D. L., Freedman, R., Schork, N. J. \& Gottesman, II (2007). Deconstructing schizophrenia: an overview of the use of endophenotypes in order to understand a complex disorder. Schizophr Bull, 33(1), 21-32.

Caspi, A., McClay, J., Moffitt, T. E., Mill, J., Martin, J., Craig, I. W., et al. (2002). Role of genotype in the cycle of violence in maltreated children. Science, 297(5582), 851-854.

Clayton, D. \& Hill, M. (1993). Wald tests. In D. Clayton \& M. Hills (Eds.), Statistical Models in Epidemiology (pp. 101-102): Oxford Science Publications.

Derom, C., Vlietinck, R., Thiery, E., Leroy, F., Fryns, J. P. \& Derom, R. (2002). The East Flanders Prospective Twin Survey (EFPTS). Twin Res, 5(5), 337-341.

Dickinson, D., Ramsey, M. E. \& Gold, J. M. (2007). Overlooking the obvious: a meta-analytic comparison of digit symbol coding tasks and other cognitive measures in schizophrenia. Arch Gen Psychiatry, 64(5), 532542.

Dollfus, S., Lombardo, C., Benali, K., Halbecq, I., Abadie, P., Marie, R. M., et al. (2002). Executive/attentional cognitive functions in schizophrenic patients and their parents: a preliminary study. Schizophr Res, 53(1-2), 93-99.

Dominguez, M., Viechtbauer, W., Simons, C., van Os, J. \& Krabbendam, L. (2009). Are psychotic psychopathology and neurocognition orthogonal? A systematic review of their associations. Psychol Bull, 135(1), 157171.

Faraone, S. V., Seidman, L. J., Kremen, W. S., Toomey, R., Pepple, J. R. \& Tsuang, M. T. (2000). Neuropsychologic functioning among the nonpsychotic relatives of schizophrenic patients: the effect of genetic loading. Biol Psychiatry, 48(2), 120-126.

Green, M. F. \& Nuechterlein, K. H. (2004). The MATRICS initiative: developing a consensus cognitive battery for clinical trials. Schizophr Res, 72(1), 1-3.

Grove, W. M., Lebow, B. S., Clementz, B. A., Cerri, A., Medus, C. \& lacono, W. G. (1991). Familial prevalence and coaggregation of schizotypy indicators: a multitrait family study. J Abnorm Psychol, 100(2), 115-121.

Heinrichs, R. W. \& Zakzanis, K. K. (1998). Neurocognitive deficit in schizophrenia: a quantitative review of the evidence. Neuropsychology, 12(3), 426-445.

Jabben, N., van Os, J., Janssen, I., Versmissen, D. \& Krabbendam, L. (2007). Cognitive alterations in groups at risk for psychosis: neutral markers of genetic risk or indicators of social disability? Acta Psychiatr Scand, 116(4), 253-262.

Jacobs, N., Kenis, G., Peeters, F., Derom, C., Vlietinck, R. \& van Os, J. (2006). Stress-related negative affectivity and genetically altered serotonin transporter function: evidence of synergism in shaping risk of depression. Arch Gen Psychiatry, 63(9), 989-996.

Janssen, I., Krabbendam, L., Bak, M., Hanssen, M., Vollebergh, W., de Graaf, R., et al. (2004). Childhood abuse as a risk factor for psychotic experiences. Acta Psychiatr Scand, 109(1), 38-45.

Keefe, R. S., Bilder, R. M., Harvey, P. D., Davis, S. M., Palmer, B. W., Gold, J. M., et al. (2006a). Baseline neurocognitive deficits in the CATIE schizophrenia trial. Neuropsychopharmacology, 31(9), 2033-2046.

Keefe, R. S., Perkins, D. O., Gu, H., Zipursky, R. B., Christensen, B. K. \& Lieberman, J. A. (2006b). A longitudinal study of neurocognitive function in individuals at-risk for psychosis. Schizophr Res, 88(1-3), 26-35.

Konings, M., Bak, M., Hanssen, M., van Os, J. \& Krabbendam, L. (2006). Validity and reliability of the CAPE: a selfreport instrument for the measurement of psychotic experiences in the general population. Acto Psychiatr Scand, 114(1), 55-61. 
Krabbendam, L., Myin-Germeys, I., Hanssen, M. \& van Os, J. (2005). Familial covariation of the subclinical psychosis phenotype and verbal fluency in the general population. Schizophr Res, 74(1), 37-41.

Larkin, W. \& Read, J. (2008). Childhood trauma and psychosis: evidence, pathways, and implications. J Postgrad Med, 54(4), 287-293.

Lawrie, S. M., Mclntosh, A. M., Hall, J., Owens, D. G. \& Johnstone, E. C. (2008). Brain structure and function changes during the development of schizophrenia: the evidence from studies of subjects at increased genetic risk. Schizophr Bull, 34(2), 330-340.

Loos, R., Derom, C., Vlietinck, R. \& Derom, R. (1998). The East Flanders Prospective Twin Survey (Belgium): a population-based register. Twin Res, 1(4), 167-175.

Morgan, C. \& Fisher, H. (2007). Environment and schizophrenia: environmental factors in schizophrenia: childhood trauma--a critical review. Schizophr Bull, 33(1), 3-10.

Nemeroff, C. B. (2004). Neurobiological consequences of childhood trauma. J Clin Psychiatry, 65 s18-28.

Read, J., Perry, B. D., Moskowitz, A. \& Connolly, J. (2001). The contribution of early traumatic events to schizophrenia in some patients: a traumagenic neurodevelopmental model. Psychiatry, 64(4), 319345.

Simons, C. J., Jacobs, N., Jolles, J., van Os, J. \& Krabbendam, L. (2007). Subclinical psychotic experiences and cognitive functioning as a bivariate phenotype for genetic studies in the general population. Schizophr Res, 92(1-3), 24-31.

Spataro, J., Mullen, P. E., Burgess, P. M., Wells, D. L. \& Moss, S. A. (2004). Impact of child sexual abuse on mental health: prospective study in males and females. Br J Psychiatry, 184, 416-421.

Spauwen, J., Krabbendam, L., Lieb, R., Wittchen, H. U. \& van Os, J. (2006). Impact of psychological trauma on the development of psychotic symptoms: relationship with psychosis proneness. Br J Psychiatry, 188, 527533.

StataCorp (2007). STATA Statistical Software: Release 10.0. Texas: College Station.

Stefanis, N. C., Hanssen, M., Smirnis, N. K., Avramopoulos, D. A., Evdokimidis, I. K., Stefanis, C. N., et al. (2002). Evidence that three dimensions of psychosis have a distribution in the general population. Psychol Med, 32(2), 347-358.

Teicher, M. H., Andersen, S. L., Polcari, A., Anderson, C. M., Navalta, C. P. \& Kim, D. M. (2003). The neurobiological consequences of early stress and childhood maltreatment. Neurosci Biobehav Rev, 27(1-2), 33-44.

van Os, J., Linscott, R. J., Myin-Germeys, I., Delespaul, P. \& Krabbendam, L. (2008a). A systematic review and meta-analysis of the psychosis continuum: evidence for a psychosis proneness-persistenceimpairment model of psychotic disorder. Psychol Med, 1-17.

van Os, J. \& Murray, R. (2008). Introduction. Schizophr Bull, 34(6), 1064-1065.

van Os, J., Rutten, B. P. \& Poulton, R. (2008b). Gene-Environment Interactions in Schizophrenia: Review of Epidemiological Findings and Future Directions. Schizophr Bull, 34(6)(6), 1066-1082.

van Os, J. \& Sham, P. (2003). Gene-environment correlation and interaction in schizophrenia. In R. Murray, P. B. Jones, E. Susser, J. Van Os \& M. Cannon (Eds.), The epidemiology of Schizophrenia. Cambridge: Cambridge University Press.

Walker, E. F. \& Diforio, D. (1997). Schizophrenia: a neural diathesis-stress model. Psychol Rev, 104(4), 667-685.

Weiser, M., van Os, J., Reichenberg, A., Rabinowitz, J., Nahon, D., Kravitz, E., et al. (2007). Social and cognitive functioning, urbanicity and risk for schizophrenia. Br J Psychiatry, 191, 320-324.

Wichers, M., Aguilera, M., Kenis, G., Krabbendam, L., Myin-Germeys, I., Jacobs, N., et al. (2007). The Catechol-OMethyl Transferase Val(158)Met Polymorphism and Experience of Reward in the Flow of Daily Life. Neuropsychopharmacology.

Wichers, M., Schrijvers, D., Geschwind, N., Jacobs, N., Myin-Germeys, I., Thiery, E., et al. (2009). Mechanisms of gene-environment interactions in depression: evidence that genes potentiate multiple sources of adversity. Psychol Med, 39(7), 1077-1086. 


CHAPTER 3

Subjective experience of cognitive failures as possible risk factor for negative symptoms of psychosis in the general population

Stefanie Pfeifer ${ }^{1}$, Jim van $0 s^{1,2}$, Manon Hanssen ${ }^{1}$, Philippe Delespaul ${ }^{1}$, Lydia Krabbendam $^{1}$

${ }^{1}$ Department of Psychiatry and Neuropsychology, South Limburg Mental Health Research and Teaching Network, EURON, Maastricht University, PO BOX 616 (VIJV1), 6200 MD Maastricht, The Netherlands

${ }^{2}$ Division of Psychological Medicine, Institute of Psychiatry, De Crespigny Park, Denmark Hill, London SE5 8AF, UK

Schizophrenia Bulletin 2009; 35 (4): 766-774. 


\section{Abstract}

Background. The aim of this study was to examine whether proneness to subjective cognitive failure (cognitive based mistakes) increases the risk for the development of symptoms of psychosis and to what degree any association was familial.

Methods. At baseline, the Cognitive Failure Questionnaire (CFQ) and the Community Assessment of Psychic Experiences (CAPE) questionnaire were administered in a general population sample of genetically related individuals $(n=755)$. Individuals scoring high $\left(>75^{\text {th }}\right.$ percentile) or average on the CAPE (between $40^{\text {th }}$ and $60^{\text {th }}$ percentile) $(n=488)$ were reinterviewed with the CAPE and Structured Interview for Schizotypy-Revised (SIS-R) at follow-up (mean interval $=7.7$ months, $\mathrm{SD}=4.8$ months).

Results. Cross-trait, within-relative analysis showed a significant association between the CFQ and the negative dimension, assessed with both the CAPE and SIS-R, whereas no association was found between the CFQ and the positive dimension. Cross-trait, betweenrelative analyses showed no association between the CFQ in one relative and any of the dimensions of the subclinical psychosis phenotype in the other relative.

Conclusion. Proneness to subjective cognitive failure possibly contributes to the development or persistence of negative symptoms and can be seen as potential risk factor for negative symptoms of psychosis. This overlap is due to individual effects rather than familial liability. 


\section{Introduction}

Subjective cognitive failures are cognitive based mistakes in processes which can normally be achieved without error (e.g., accidentally throwing away a new pen and keeping the old one). They refer to common errors that occur in everyday life, such as memory slips (e.g., absent-mindedness), attention slips (e.g., to fail to notice something relevant), and action slips (e.g., to perform an unintended action) (Broadbent, et al., 1982; Wallace, et al., 2002). Underlying this definition is the assumption that an individual is capable of performing a certain task but fails because something else interferes with completion of the task (Wallace, 2004). This account of the subjective experience of a cognitive failure is in line with studies that conclude that cognitive failure is unrelated to objective cognitive test performance (Rabbitt \& Abson, 1990; Wallace, 2004). Proneness to subjective cognitive failure seems to be a stable characteristic, which is expressed across a variety of situations and can be assessed reliably using self-report questionnaires, such as the Cognitive Failures Questionnaire (CFQ) (Broadbent, et al., 1982). The CFQ measures self reported frequency of subjective cognitive failures in daily life. Individuals scoring high on the CFQ report higher levels of anxiety and depression, both cross-sectionally (Merckelbach, et al., 1996) and longitudinally (Power, 1988). This effect may be particularly evident in combination with exposure to stressful environments (Broadbent, et al., 1982), suggesting that individuals scoring high on the CFQ are less successful at developing active coping strategies in dealing with stress (Broadbent, et al., 1982; Power, 1988). The lack of active coping strategies may be explained on the basis of a less effective management of attentional capacity, possibly putting individuals at risk of developing depressive symptoms (Broadbent, et al., 1982).

It has also been suggested that a high score on the CFQ reflects a vulnerability to automatic intrusions that interfere with conscious processing. Under stress, these normally benign intrusions may become malevolent (Power, 1988). This account of subjective cognitive failure is reminiscent of the role of automatic cognitive biases that laboratory experiments have shown to operate in anxiety and depression (Merckelbach, et al., 1996; Williams, et al., 1996).

Cognitive accounts of psychosis have increasingly recognised that processes relevant to anxiety and depression may also play a role in psychotic symptom formation (Blackwood, et al., 2001; Freeman, et al., 2001; Garety, et al., 2001; Morrison, 2001; Taylor \& John, 
2004). For example, individuals with persecutory delusions show an attention and memory bias for threatening information (Fear, et al., 1996; Kaney, et al., 1992) and individuals with hallucinations experience more intrusive thoughts and find these thoughts more distressing and uncontrollable than psychiatric and healthy control groups (Morrison \& Baker, 2000). If these cognitive mechanisms which are relevant to anxiety and depression likely also apply to psychotic symptom formation (Blackwood, et al., 2001; Taylor \& John, 2004), it can be suggested that the associations between subjective cognitive failures on the one hand and anxiety/depression and psychosis on the other can be explained on the basis of a shared mechanism.

Also, individuals with psychosis may have an increased sensitivity to daily life stress (MyinGermeys, et al., 2001) as well as less effective coping strategies (Bak, et al., 2003; Bak, et al., 2001). These processes may well be captured by the construct of cognitive failure but so far there have been no studies investigating the association between subjective cognitive failure and psychosis. There is evidence that subjective experience of cognitive disturbances predict psychotic symptoms (Bechdolf, et al., 2002; Klosterkotter, et al., 1996; Klosterkotter, et al., 1997; Pukrop, et al., 2006) but this refers to the experience of basic symptoms which are phenomenologically close to the positive symptoms of psychosis.

Whereas the lifetime prevalence of psychotic disorders is estimated between $0.5 \%$ to $3 \%$ (Kendler, et al., 1996; Perala, et al., 2007; Rossler, et al., 2005), there is a growing body of literature suggesting that this may represent only a minor selection of the real prevalence of psychotic symptoms in the general population (Johns \& van Os, 2001; Kendler, et al., 1996; van Os, et al., 2000). These attenuated symptoms of psychosis share many of the epidemiologic and phenomenological characteristics of the clinical disorder, supporting the notion of continuity between the clinical and the subclinical phenotype (Johns $\&$ van Os, 2001).

Longitudinal general population studies are well suited to test the role of cognitive mechanisms in psychotic symptom formation, since the association with de novo emergence of symptoms can be assessed and any confounding influence of characteristics related to patient status can be avoided. The first aim of the current study was therefore to investigate the relationship between subjective cognitive failure and subclinical symptoms of psychosis in a longitudinal general population study. 
The attenuated symptoms of psychosis tend to cluster within families, both in nonpsychotic relatives of patients with schizophrenia (Grove, et al., 1991; Grube, et al., 1998) and in the general population (Hanssen, et al., 2006; Linney, et al., 2003; Stefanis, et al., 2002), indicative of familiality of the subclinical psychosis phenotype, as observed for the clinical phenotype, which can be largely explained by the effect of shared genes (Johns \& van Os, 2001). Similarly, a substantial part of the inter-individual variation in proneness to subjective cognitive failure can be attributed to genetic factors (Boomsma, 1998). This raises the issue of both traits vary as part of the same underlying cause, similar to what has been shown for neurocognition on the one hand and subclinical psychosis on the other (Krabbendam, et al., 2005b; Simons, et al., 2007). This was investigated in the second part of this study.

The Community Assessment of Psychic Experiences (CAPE) questionnaire and the Structured Interview for Schizotypy-Revised (SIS-R) are two useful validated and replicated instruments to assess the subclinical psychosis phenotype (Konings, et al., 2006; Vollema \& Ormel, 2000). In the context of the current article, the term 'psychosis' refers to the full range of signs/symptoms/and deviant experiences observed in cases who receive a 'psychotic' diagnosis. The positive, the negative, and the depressive dimension of the CAPE and the positive, the negative, and the disorganization dimension of the SIS-R are separate dimensions of the subclinical psychosis phenotype, and there is increasing evidence that these dimensions can be found not only in patients with psychotic disorders (Blanchard \& Cohen, 2006; Grube, et al., 1998; Peralta \& Cuesta, 2001) but also in the general population (Stefanis, et al., 2002), showing a continuous distribution (Johns \& van Os, 2001).

Two approaches were used to investigate the relationship between subjective cognitive failure and subclinical psychosis. First, in a cross-trait, within-relative approach, the hypothesis was tested that subjective cognitive failures are associated with the different dimensions of the subclinical psychosis phenotype. Second, in a cross-trait, betweenrelative approach, it was examined whether subjective cognitive failures in one relative are associated with the subclinical psychosis phenotype in the other. 


\section{Method}

\section{Procedure and Sample}

The Continuum of Mental Disorders Study is a longitudinal family study of the general population in the city of Sittard, The Netherlands (Hanssen, et al., 2006). This study has two measurement points: T1 and T2, with a mean interval of 7.7 month (SD= 4.8 month, range: 1 - 26 month) between these two measurement occasions.

In order to recruit a representative sample of the general population, 4589 participants between 36 and 65 years were randomly selected and sent a letter in which they and their family members were asked to participate. The total general population sample for T1 comprised 768 participants aged 17 - 77 years, pertaining to 116 families. Taking into consideration every possible family relationship between subjects, $61.0 \%$ of the samples were first-degree relatives, $18.2 \%$ second-degree, $6.6 \%$ third-degree, and $0.4 \%$ were fourth-degree relatives; $13.8 \%$ of the subjects were married or partners. In accordance with the local medical ethical committee, written inform consent was received from all participants.

At T1, the CFQ and the CAPE were administered. All participants with an average (between $40^{\text {th }}$ and $60^{\text {th }}$ percentile) and a high (above $75^{\text {th }}$ percentile) score on the CAPE at T1 and their family members were asked to participate at T2.

This strategy was aimed at oversampling of individuals with higher levels of psychosis, thus increasing statistical power, while at the same time ensuring that the sample included sufficient individuals with 'average' levels of the subclinical psychosis phenotype so as to have sufficient variation along a hypothesized continuum of psychosis.

At T2, participants filled in the CAPE questionnaire, and trained psychologists administered the Dutch version of the SIS-R (Kendler, et al., 1989; Vollema \& Ormel, 2000).

The risk set consisted of all subjects who had completed the CFQ at T1 and (a) the CAPE at T1 $(n=755)$, (b) the CAPE at T2 $(n=501)$ and (c) the SIS-R at T2 $(n=488)$.

\section{Instruments}

The CAPE (Stefanis, et al., 2002) is a self-report questionnaire rating attenuated affective and nonaffective psychotic experiences (for detailed information of the CAPE see http://www.cape42.homestead.com/). The CAPE measures, on a dimensional scale, frequency of, as well as distress associated with, these subclinical psychotic experiences. 
The frequency score is measured on a four-point scale from 'never (1)', 'sometimes (2)', 'often (3)', to 'nearly always (4)'. The degree of distress associated with the subclinical psychotic experience is also measured on a four-point scale with labels ranging from 'not distressed (1)', 'a bit distressed (2)', 'quite distressed (3)', to 'very distressed (4)'. The CAPE includes dimensions of positive (20 items), negative (14 items), and depressive ( 8 items) psychotic symptoms associated with the subclinical psychosis phenotype in the general population (see Appendix for example of the 14 negative items of the CAPE). The CAPE provides a total score per dimension by adding up the scores on the frequency question, yielding a total frequency score, and adding up the scores on the distress question, yielding a distress score. A weighted score was calculated for each frequency and for each distress score to account for partial non-response (Konings, et al., 2006; Stefanis, et al., 2002).

Furthermore, a validated Dutch translation of the CFQ was administered (Merckelbach, et al., 1996). The CFQ is a self-report questionnaire consisting of 25-items and comprising four main subscales: absent-mindedness, social interactions, names and words, and orientation (Wallace, 2004). On a five-point subscale, the participants had to indicate how often they experience subjective cognitive failures. The scale ranges from 'never $(0)^{\prime}$, 'very rarely (1)', 'occasionally (2)', 'quite often (3)', to 'very often (4)'. Total scores for the CFQ ranged from 0 to 100 . A higher score on the CFQ indicates more subjectively experienced cognitive failures.

The SIS-R is a structured interview and is designed to measure the symptoms and signs of the positive, negative, and disorganization dimensions of the subclinical psychosis phenotype. Items can be scored on a four-point scale from absent (score 0) to severe (score 3 ). Positive schizotypy covers the symptoms referential thinking, magical ideation, illusions and suspiciousness (6 items in total). Negative schizotypy contains the symptoms social isolation, social anxiety, introversion, restricted affect, referential thinking, and suspiciousness ( 8 items in total). Disorganization schizotypy encompasses the signs goaldirectness of thinking, loosening of associations, and oddness ( 3 items in total).

\section{Analyses}

All analyses were carried out using STATA version 9.2 (StataCorp, 2006).

First, cross-trait, within-relative linear regression analyses were conducted to investigate, within subjects, the longitudinal association between total score on the CFQ at T1 and 
each dimension of the subclinical psychosis phenotype at T2. Robust estimates of variance were used, which allow observations that are not independent across groups (i.e., families). All analyses were adjusted for the a priori demographical confounders sex, age, level of education and current drug use. Any significant association was additionally adjusted for the other two remaining dimensions of the subclinical psychosis phenotype in order to examine the degree to which the association was reducible to overlap with the other subclinical psychosis dimensions. Analyses of distress associated with the subclinical psychosis dimensions of the CAPE questionnaire were additionally adjusted for the corresponding frequency score. Effect sizes were expressed as the standardized regression coefficient (beta). Linear combination of coefficients (LINCOM procedure) was additionally performed in order to test the difference between the different coefficients of the various subclinical dimensions.

In order to examine any specific patterns of associations with psychosis, the four subscales of the CFQ were also entered separately in the regression models.

In order to investigate whether any association between the CFQ total score at the T1 measurement and the subclinical psychosis phenotype (measured with the CAPE at T2) was independent of baseline presence of subclinical psychosis symptoms, all associations were adjusted for the corresponding subclinical psychosis dimensions measured with the CAPE at the T1 measurement.

Second, cross-trait, between-relative analyses were conducted in order to examine the associations between the CFQ total score in relative 1 and dimensions of the subclinical psychosis phenotype in relative 2 . A data set was generated that contained all possible pairwise relationships between subjects, either unrelated (i.e., no shared genes and not married) or related (either $6.25 \%, 12.5 \%, 25 \%$, or $50 \%$ shared genes). Married pairs were not included in this data set, in order to avoid confounding due to assortative mating. Interactions were fitted between shared genes, dichotomously defined as any degree of sharing and dimensions of the subclinical psychosis phenotype in relative 2 , using the CFQ total score in relative 1 as the outcome variable. All analyses were adjusted for age, sex, level of education and drug use of both relative 1 and relative 2 and additionally for the corresponding dimension of the subclinical psychosis phenotype in relative 1 as well as the CFQ total score in relative 2. Again, analyses of distress associated with the subclinical psychosis dimensions of the CAPE questionnaire were additionally adjusted for the corresponding frequency dimension score of relative 1 as well as of relative 2 . Robust 
estimates of variance were used, and effect sizes were expressed as the standardized regression coefficient.

\section{Results}

\section{Sample}

At $\mathrm{T} 1$, the sample consisted of 755 participants (62.0\% female) who completed the CAPE questionnaire. The mean age was 46.3 years $(S D=12.7$, range: $15-77)$. Mean education level was 4.7 (1.7) on an eight-point scale ranging from primary education to university degree. Self-reported current drug use was present in 4.2\% (range: 0 - 6 drugs used). Mean total score on the CFQ was 32.7 ( $S D=11.2$ ). The means of the weighted positive, negative and depressive dimensions of the CAPE were $1.42(S D=0.26), 1.62(S D=0.37)$ and $1.72(S D=0.41)$, respectively. At $T 2$, the sample consisted of 501 participants $(60.7 \%$ female) (of these, 488 participants (60.4\% female) completed the SIS-R). The means of the weighted positive, negative, and depressive dimensions of the CAPE were $1.16(S D=0.18)$, $1.56(S D=0.34)$ and $1.61(S D=0.35)$, respectively. Mean score for the SIS-R positive dimension was $2.15(S D=2.21)$, for the negative dimension $2.84(S D=2.77)$ and for the disorganization dimension $0.10(S D=0.52)$.

\section{Cross-trait, within-relative analysis}

Self-reported subclinical psychosis dimensions. The results of the longitudinal cross-trait, within-relative analyses showed a significant association between the CFQ total score at T1 and frequency of the positive, the negative, and the depressive dimensions of the CAPE at T2 $(B=0.262, p=0.000 ; b=0.532, p=0.000 ; B=0.540, p=0.000$, respectively). Furthermore, a significant association was also found between the CFQ total score at T1 and distress of the positive, the negative, and the depressive dimensions of the CAPE at T2 ( $b=0.238, p=0.000 ; b=0.221, p=0.000 ; b=0.105, p=0.042$, respectively). The association between the CFQ total score and the frequency of the negative and depressive dimensions was significantly stronger than the association between the CFQ total score and the frequency of the positive dimension $(t=4.00, p=0.000,95 \%$ confidence interval $(\mathrm{Cl}): 1.83-$ 5.37; and $\mathrm{t}=4.02, \mathrm{p}=0.000,95 \% \mathrm{Cl}: 1.89-5.22$, respectively). No difference was found between the frequency of the negative dimension coefficient and the depressive dimension coefficient $(p=0.966)$. After an additional adjustment for the other two 
remaining dimensions of the subclinical psychosis phenotype, only the association between the CFQ total score at T1 and the frequency and distress of the negative dimension and frequency of the depressive dimension at $\mathrm{T} 2$ remained significant (Table 1). The association between the CFQ total score and the negative dimension at T2 was reduced but remained significant even after adjustment for the negative dimension at T1 (frequency: $B=0.23, p=0.000$; distress: $B=0.112, p=0.05$ ).

Similarly, the association between the CFQ total score and the frequency of the depressive dimension at $\mathrm{T} 2$ was reduced but remained significant after adjustment for the depressive dimension at T1 (frequency: $b=0.24, p=0.000$ ), whereas the association between the CFQ total score and distress disappeared (distress: $6=-0.016, p=0.771$ ).

Comparable to the results of the total CFQ score, the analyses of the four CFQ subscales separately showed a similar pattern of results (data not shown).

Interview-based subclinical psychosis dimensions. The CFQ total score at T1 was significantly associated with the positive dimension and the negative dimension of the SIS$\mathrm{R}$ at $\mathrm{T} 2(B=0.188, \mathrm{p}=0.002$; and $B=0.355, \mathrm{p}=0.000$, respectively) but not with the disorganization dimension of the SIS-R at T2 $(B=0.012, p=0.827)$.

The association between the CFQ total score and the negative dimension was significantly stronger than the association between the CFQ total score and the positive dimension ( $t=$ 3.38, $p=0.001,95 \% \mathrm{Cl}: 0.65-2.456)$ and the disorganization dimension $(t=2.52, p=0.012$, $95 \% \mathrm{Cl}: 0.667-5.455)$. No difference between the association of the CFQ total score and the positive dimension and the CFQ total score and the disorganization dimension was found $(p=0.218)$.

After adjustment of the subclinical psychosis dimensions for each other, only the association between the CFQ total score and the negative dimension remained significant (see Table 1).

Again, comparable to the results of the total CFQ score, the analyses of the four CFQ subscales separately showed a similar pattern of results (data not shown).

\section{Cross-trait, between-relative analyses}

For the cross-trait, between-relative analyses, only participants who participated with their family members at both $\mathrm{T} 1$ and $\mathrm{T} 2$ were included (566 pairs pertaining to 72 families). In the model of the CFQ total score outcome at $\mathrm{T} 1$, there was no significant 
interaction between the variable shared genes and the frequency of CAPE positive $(B=$ $0.0001, \mathrm{t}=0.06, \mathrm{p}=0.949)$, negative $(B=0.0009, \mathrm{t}=1.00, \mathrm{p}=0.319)$, and depressive dimensions $(b=0.0001, \mathrm{t}=0.98, \mathrm{p}=0.326)$ at T2 nor with the SIS-R positive $(b=-0.0018, \mathrm{t}=-$ $0.64, p=0.519)$, negative $(b=-0.0029, t=-0.92, p=0.356)$, and disorganization dimensions $(b=-0.0012, t=-0.74, p=0.458)$ at $T 2$. Similarly, there was no interaction between the variable shared genes and the distress of the CAPE positive $(B=-0.003, t=-1.60, p=0.109)$, negative $(B=-0.0004, \mathrm{t}=-0.317, \mathrm{p}=0.751)$, and depressive dimensions $(B=-0.0002, \mathrm{t}=-$ $0.184, \mathrm{p}=0.854)$ at $\mathrm{T} 2$.

\section{Discussion}

This study was, to our knowledge, the first general population study, examining the association between proneness to everyday subjective cognitive failures, assessed with the CFQ, a self-report questionnaire, and the subclinical psychosis phenotype within and between-relatives. Overall, the results of the cross-trait, within-relative analyses showed a strong and robust positive association between subjective cognitive failure at baseline and frequency of negative symptoms at follow-up, both self-reported (assessed with the CAPE) and interview-based (assessed with the SIS-R). A higher level of subjective cognitive failure at baseline also increased the distress associated with these negative symptoms at followup. In line with previous literature (Merckelbach, et al., 1996; Power, 1988), subjective cognitive failure also showed a positive association with the frequency of self-reported depressive symptoms, although the association with distress related to depressive symptoms was a result of the overlap with the negative dimension. The associations between subjective cognitive failure and the positive dimension, both self-reported and interview-based, were reducible to the other subclinical psychosis dimensions. No association was found with the interview-based disorganization dimension.

The associations between subjective cognitive failure and the negative and depressive dimensions were reduced but not nullified after adjustment for the remaining corresponding dimensions of the baseline measurement. This may suggest that proneness to subjective cognitive failure contributes to the development or persistence of negative and depressive symptoms, independent of their baseline presence, and can be seen as a potential risk factor for developing negative and depressive symptoms associated with 
psychosis. This rules out the possibility that reverse causality (i.e., symptoms interfere with cognitive capacity, and, therefore, more subjective cognitive failures in daily live develop) (Merckelbach, et al., 1996) can explain the observed association.

In the cross-trait, between-relative analyses, no evidence was found for familial continuity between subjective cognitive failure and the subclinical psychosis dimensions, suggesting that the overlap within-relatives was due to individual rather than familial liability. Because the results of the cross-trait, between-relative analyses do not substantiate a genetic transmission of the observed overlap between the phenotypes, an alternative explanation would be that psychological mechanisms lie at the basis of the association. Speculatively, individuals with a high level of subjective cognitive failures in daily life may try to avoid situations where the chance of experiencing subjective cognitive failures is increased, e.g., in social contacts. Possibly, negative symptoms may develop partly as a result of this avoidance behaviour.

Individuals prone to subjective cognitive failure were also more prone to report distress associated with the negative symptoms, independent of their frequency. This is important because recent insights suggest that it is the distress associated with the subclinical psychotic experience which causes transition to need for care status, rather than the mere experience itself (Hanssen, et al., 2005; Krabbendam, et al., 2005a; Morrison, 2001). The fact that individuals with negative symptoms experienced more distress associated with subjective cognitive failures may seem contradictory because negative symptoms are traditionally associated with blunting of affect. However, only one study actually assessed to what degree negative symptoms are truly associated with emotional reactivity rather than the observable emotional expression. This study, using momentary assessment methodology, showed that patients in fact experienced more intense and more variable negative emotions than controls. In addition, no difference in patterns of affect was found between the blunted and the nonblunted schizophrenia subgroups (Myin-Germeys, et al., 2000). These findings indicate that there is a difference between negative symptoms as the observable expression of emotions (in virtually all patients associated to a degree with the effects of D2-blocking drugs) and negative symptoms as the experience of emotions. Those at risk of negative symptoms may show greater emotional reactivity that is associated with the clinical phenotype of psychotic disorder.

Studies investigating neurocognitive test performance have similarly reported covariation with subclinical psychosis, both in relatives (Grove, et al., 1991; Nuechterlein, et al., 2002) 
and in the general population (Dinn, et al., 2002; Krabbendam, et al., 2005b). There is evidence that the overlap between neurocognition and subclinical psychosis has a common genetic basis (Krabbendam, et al., 2005b; Simons, et al., 2007). The current findings did not point to a shared genetic basis for the overlap between subjective cognitive failure and subclinical psychosis. However, this does not point to a discrepancy between this study and previous findings because the construct of subjective cognitive failure assumes that it is unrelated to neurocognitive functioning (Wallace, 2004). More specifically, the findings suggest that the CFQ is associated with the negative domain of psychometric psychosis risk within individuals but not within families. This is an important difference with measures of neurocognition, alterations of which are transmitted together with risk for psychosis from one generation to another (Toulopoulou, et al., 2007). Earlier studies have indicated that the CFQ, as a measure of the experience of cognitive functioning, is not associated with actual cognitive functioning, as measured by tests of neurocognition (Ponds, 1998; Rabbitt \& Abson, 1990). Therefore, the question rises what area of individual-specific rather than family-specific psychosis liability the CFQ is tapping into. Several related possibilities arise. The fact that the CFQ assesses experience of cognitive functioning indicates that as a construct it is closer to the process of metacognition (thinking about one's cognition) rather than the process of cognitive function itself. Previous work has shown that metacognitive factors are important in the development of symptoms associated with psychotic disorder (Bak, et al., 2005; Linney \& Peters, 2007; Morrison, et al., 2007). In agreement with this is work showing that the CFQ is associated with factors such as anxiety and depression or sensitivity to stress (Broadbent, et al., 1982; Merckelbach, et al., 1996). Therefore, the current findings of the CFQ suggest that the area of liability involved may be closer to the domain of social cognition than the domain of neurocognition, which do not appear to be strongly correlated with each other (van Hooren, et al., 2008). The fact that the liability measured with the CFQ is individual-specific rather than shared with relatives in the same family suggests that this aspect of metacognition is associated with individual-level environmental exposures such as developmental trauma (Bak, et al., 2005; Glaser, et al., 2006).

According to cognitive models, cognitive disturbances contribute to the development and persistence of psychopathology (MacLeod \& Hagan, 1992; Morrison, 2001; Williams, et al., 2007). Thus, in panic disorder, the tendency to misinterpret bodily sensations in a 
catastrophic manner is maintained by selective attention to idiosyncratic threat cues and avoidance behaviour that prevents disconfirmation of threat. Likewise, hallucinations and delusions may be conceptualised as intrusive thoughts, which are interpreted in a malevolent way, this interpretation leading to the associated distress and disability (Morrison, 2001). In this light, the fact that the association between subjective cognitive failure and the positive dimension was entirely due to the overlap with the negative and the depressive dimensions of psychosis was not expected. A possible explanation for this finding is that expression of the symptom dimensions of psychosis in the general population is much more attenuated than in clinical samples. This makes it much more difficult to measure the dimensions of psychosis sensitively and to distinguish between them. Accordingly, correlations between the dimensions are larger in the general population compared with clinical samples (Stefanis, et al., 2002). Another possible explanation is that the CFQ is not sensitive enough to detect differential cognitive biases associated with specific forms of psychopathology. Although the CFQ contains three separate categories (attention, memory, and action) which are supported by factor analyses, all factors are highly related (Wallace, 2004) and many items may refer to phenomena involving aspects of all three areas (e.g., 'Do you find you forget appointments?') (Merckelbach, et al., 1996). This is in line with earlier explanations that the score on the CFQ reflects limitations of a general cognitive capacity, which increase in stressful situations (Broadbent, et al., 1982). Finally, subjective cognitive failures were assessed with a self-report instrument that may have low sensitivity to detect specific forms of psychopathology, particularly those associated with positive symptoms of psychosis. The lack of sensitivity may explain the absence of an association between the CFQ and positive psychotic symptoms.

\section{Limitations}

Results should be interpreted in the context of several limitations. First, the CFQ reflects a general tendency to everyday subjective cognitive failure. Therefore, it was not possible to elucidate associations between specific cognitive disturbances and the dimensions of the subclinical psychosis phenotype. In order to examine specific cognitive biases, the CFQ as a self-report measure is not suitable and experimental tasks would be required.

Second, the present study could not rule out the possibility that the association between subjective cognitive failure and negative psychotic symptoms is influenced by one 
common factor, e.g., neuroticism. This may be particularly relevant for associations with the SIS-R negative dimension because the SIS-R does not distinguish between social isolation based on apprehension and social isolation resulting from low social drive.

Third, although some of the explanations offered for the findings involve differential exposure to stress, no measures of stressful circumstances were available for analysis. There is evidence, however, that an increase in stress in daily life in patients with psychotic disorders is associated with an increase of psychotic symptoms, independent of the amount of cognitive impairment of the patients (Myin-Germeys, et al., 2002).

Fourth, although it is theoretically plausible to interpret proneness to subjective cognitive failure as a causal risk factor in the development or persistence of negative symptoms of psychosis, this study demonstrated a statistical association and therefore could not prove causality.

Fifth, as a result of carefully conducted analyses in earlier work (Vollema \& Ormel, 2000), the scales of the SIS-R positive and the negative dimensions show a small degree of item overlap. Although this may seem contradictory, earlier work has established that at the level of the general population, higher correlations between the different dimensions may occur than seen in patient samples where correlations are lower, although not orthogonal (Peralta \& Cuesta, 1999; Peralta, et al., 1997; Peralta, et al., 2001). The reason for the observation of higher correlations in the general population is that in non-clinical samples, expression of psychotic phenomena is much more attenuated, making it more difficult to discriminate between different domains. In contrast, in clinical samples, it is possible that one main symptom overshadows the other symptoms, resulting in reduced correlations between the dimensions (Peralta \& Cuesta, 1999; Stefanis, et al., 2002). The item overlap in SIS-R dimensions should not detract from the overall face validity and clearly different item content of the positive and negative scales. An additional post-hoc regression analysis of the 'affect expressive' (referential thinking, suspiciousness, and social anxiety) and the 'affect restricted' (restricted affect, introversion, and social isolation) items of the negative schizotypy dimension also showed that both contributed equally to the observed association between the CFQ and the negative dimension of the SIS-R (affect expressive: $B=0.1354, p=0.003$; affect restrictive: $B=0.1348, p=0.000$ ). These results confirm that the extremes of the clinical phenotype in patients with psychotic disorder do not separate as distinctly when the phenotype is examined at lower levels of intensity in non-patients (Peralta \& Cuesta, 1999; Stefanis, et al., 2002). In addition, the schizotypy literature has 
shown that while the dimensions of schizotypy resemble those of schizophrenia, they also contain items that are not typically associated with the symptoms of schizophrenia (Gruzelier, 1996; Lewandowski, et al., 2006; Mata, et al., 2003; Vollema \& Hoijtink, 2000; Vollema \& van den Bosch, 1995).

Sixth, the relatively small sample size could account for the absence of significant effects in the cross-trait, between-relative analyses. However, the results were not even suggestive of such an effect, whereas a previous study in the same sample did yield evidence for familial continuity between measures of neurocognition and subclinical psychosis (Krabbendam, et al., 2005b).

Finally, our findings are partially based on self-report data, which may yield less precise results, especially in the case of psychosis. However, the fact that the association with the negative dimension was apparent in both analyses suggests that the self-report questionnaire and the interview instrument were equally able to capture this relationship and provides support for the validity of the findings.

\section{Funding}

Sanofi-Synthelabo Group (2002 Research Prize of the Association of European Psychiatrists). 


\section{Appendix}

Items of the negative dimension of the CAPE:

Do you ever feel that you are not a very animated person?

Do you ever feel that you are not much of a talker when you are conversing with other people?

Do you ever feel that you experience few or no emotions at important events?

Do you ever feel that you have no interest to be with other people?

Do you ever feel that you are lacking in motivation to do things?

Do you ever feel that you are lacking in energy?

Do you ever feel that your mind is empty?

Do you ever feel that you are spending all your days doing nothing?

Do you ever feel that your feelings are lacking in intensity?

Do you ever feel that you are lacking in spontaneity?

Do you ever feel that your emotions are blunted?

Do you ever feel that you are neglecting your appearance or personal hygiene?

Do you ever feel that you can never get things done?

Do you ever feel that you have only few hobbies or interests? 
Table 1. Cross-trait, within-relative analysis of the separate associations between the different dimensions of the subclinical psychosis phenotype at $\mathrm{T} 2$ and subjective cognitive failures at $\mathrm{T} 1$.

\begin{tabular}{|c|c|c|c|c|}
\hline Subclinical psychosis dimension & $\mathrm{B}(\mathrm{SE})$ & $95 \% \mathrm{Cl}$ & $P$ & $\begin{array}{l}\text { Standardized } \\
\text { Beta }(B)\end{array}$ \\
\hline \multicolumn{5}{|l|}{ Frequency $^{\mathrm{a}}$} \\
\hline CAPE positive dimension & $-0.058(0.507)$ & $-1.056,0.940$ & 0.909 & -0.005 \\
\hline CAPE negative dimension & $3.542(0.624)$ & $2.313,4.771$ & 0.000 & 0.312 \\
\hline CAPE depressive dimension & $3.494(0.632)$ & $2.250,4.739$ & 0.000 & 0.308 \\
\hline SIS-R positive schizotypy & $-0.017(0.296)$ & $-0.599,0.566$ & 0.955 & -0.003 \\
\hline SIS-R negative schizotypy & $1.536(0.275)$ & $0.996,2.077$ & 0.000 & 0.373 \\
\hline SIS-R disorganization & $-1.524(1.160)$ & $-3.809,0.760$ & 0.190 & -0.070 \\
\hline \multicolumn{5}{|l|}{ Distress $^{\mathrm{b}}$} \\
\hline CAPE positive dimension & $0.453(0.584)$ & $-0.697,1.604$ & 0.438 & 0.0409 \\
\hline CAPE negative dimension & $2.334(0.822)$ & $0.716,3.953$ & 0.005 & 0.2088 \\
\hline CAPE depressive dimension & $-0.593(0.718)$ & $-2.008,0.821$ & 0.410 & -0.0536 \\
\hline
\end{tabular}

Notes: All associations adjusted for sex, age, education, drug use and the other subclinical psychosis dimensions. $\mathrm{Cl}$, confidence interval; SE, standard error; CAPE, Community Assessment of Psychic Experiences; SIS-R, Structured Interview for Schizotypy-Revised.

${ }^{a}$ Adjusted for the frequency score of the other remaining subclinical dimensions.

${ }^{\mathrm{b}}$ Adjusted for the distress score of the other remaining subclinical psychosis dimensions and for the corresponding frequency score. 


\section{References}

Bak, M., Krabbendam, L., Janssen, I., de Graaf, R., Vollebergh, W. \& van Os, J. (2005). Early trauma may increase the risk for psychotic experiences by impacting on emotional response and perception of control. Acta Psychiatr Scand, 112(5), 360-366.

Bak, M., Myin-Germeys, I., Hanssen, M., Bijl, R., Vollebergh, W., Delespaul, P., et al. (2003). When does experience of psychosis result in a need for care? A prospective general population study. Schizophr Bull, 29(2), 349-358.

Bak, M., van der Spil, F., Gunther, N., Radstake, S., Delespaul, P. \& van Os, J. (2001). MACS-II: does coping enhance subjective control over psychotic symptoms? Acta Psychiatr Scand, 103(6), 460-464.

Bechdolf, A., Schultze-Lutter, F. \& Klosterkotter, J. (2002). Self-experienced vulnerability, prodromal symptoms and coping strategies preceding schizophrenic and depressive relapses. Eur Psychiatry, 17(7), 384-393.

Blackwood, N. J., Howard, R. J., Bentall, R. P. \& Murray, R. M. (2001). Cognitive neuropsychiatric models of persecutory delusions. Am J Psychiatry, 158(4), 527-539.

Blanchard, J. J. \& Cohen, A. S. (2006). The structure of negative symptoms within schizophrenia: implications for assessment. Schizophr Bull, 32(2), 238-245.

Boomsma, D. I. (1998). Genetic analysis of cognitive failures (CFQ): a study of Dutch adolescent twins and their parents. Eur J Personality, 12(5), 321-330.

Broadbent, D. E., Cooper, P. F., FitzGerald, P. \& Parkes, K. R. (1982). The Cognitive Failures Questionnaire (CFQ) and its correlates. Br J Clin Psychol, 21(Pt1), 1-16.

Dinn, W. M., Harris, C. L., Aycicegi, A., Greene, P. \& Andover, M. S. (2002). Positive and negative schizotypy in a student sample: neurocognitive and clinical correlates. Schizophr Res, 56(1-2), 171-185.

Fear, C., Sharp, H. \& Healy, D. (1996). Cognitive processes in delusional disorders. Br J Psychiatry, 168(1), 61-67.

Freeman, D., Garety, P. A. \& Kuipers, E. (2001). Persecutory delusions: developing the understanding of belief maintenance and emotional distress. Psychol Med, 31(7), 1293-1306.

Garety, P. A., Kuipers, E., Fowler, D., Freeman, D. \& Bebbington, P. E. (2001). A cognitive model of the positive symptoms of psychosis. Psychol Med, 31(2), 189-195.

Glaser, J. P., van Os, J., Portegijs, P. J. \& Myin-Germeys, I. (2006). Childhood trauma and emotional reactivity to daily life stress in adult frequent attenders of general practitioners. J Psychosom Res, 61(2), 229-236.

Grove, W. M., Lebow, B. S., Clementz, B. A., Cerri, A., Medus, C. \& lacono, W. G. (1991). Familial prevalence and coaggregation of schizotypy indicators: a multitrait family study. J Abnorm Psychol, 100(2), 115-121.

Grube, B. S., Bilder, R. M. \& Goldman, R. S. (1998). Meta-analysis of symptom factors in schizophrenia. Schizophr Res, 31(2-3), 113-120.

Gruzelier, J. H. (1996). The factorial structure of schizotypy: Part I. Affinities with syndromes of schizophrenia. Schizophr Bull, 22(4), 611-620.

Hanssen, M., Bak, M., Bijl, R., Vollebergh, W. \& van Os, J. (2005). The incidence and outcome of subclinical psychotic experiences in the general population. Br J Clin Psychol, 44(Pt 2), 181-191.

Hanssen, M., Krabbendam, L., Vollema, M., Delespaul, P. \& Van Os, J. (2006). Evidence for instrument and familyspecific variation of subclinical psychosis dimensions in the general population. $J$ Abnorm Psychol, 115(1), 5-14

Johns, L. C. \& van Os, J. (2001). The continuity of psychotic experiences in the general population. Clin Psychol Rev, 21(8), 1125-1141.

Kaney, S., Wolfenden, M., Dewey, M. E. \& Bentall, R. P. (1992). Persecutory delusions and recall of threatening propositions. Br J Clin Psychol, 31(Pt 1), 85-87.

Kendler, K. S., Gallagher, T. J., Abelson, J. M. \& Kessler, R. C. (1996). Lifetime prevalence, demographic risk factors, and diagnostic validity of nonaffective psychosis as assessed in a US community sample. The National Comorbidity Survey. Arch Gen Psychiatry, 53(11), 1022-1031.

Kendler, K. S., Lieberman, J. A. \& Walsh, D. (1989). The Structured Interview for Schizotypy (SIS): a preliminary report. Schizophr Bull, 15(4), 559-571.

Klosterkotter, J., Ebel, H., Schultze-Lutter, F. \& Steinmeyer, E. M. (1996). Diagnostic validity of basic symptoms. Eur Arch Psychiatry Clin Neurosci, 246(3), 147-154.

Klosterkotter, J., Schultze-Lutter, F., Gross, G., Huber, G. \& Steinmeyer, E. M. (1997). Early self-experienced neuropsychological deficits and subsequent schizophrenic diseases: An 8-year average follow-up prospective study. Acta Psychiatr Scand, 95(5), 396-404.

Konings, M., Bak, M., Hanssen, M., van Os, J. \& Krabbendam, L. (2006). Validity and reliability of the CAPE: a selfreport instrument for the measurement of psychotic experiences in the general population. Acta Psychiatr Scand, 114(1), 55-61. 
Krabbendam, L., Myin-Germeys, I., Hanssen, M., de Graaf, R., Vollebergh, W., Bak, M., et al. (2005a). Development of depressed mood predicts onset of psychotic disorder in individuals who report hallucinatory experiences. Br J Clin Psychol, 44(Pt 1), 113-125.

Krabbendam, L., Myin-Germeys, I., Hanssen, M. \& van Os, J. (2005b). Familial covariation of the subclinical psychosis phenotype and verbal fluency in the general population. Schizophr Res, 74(1), 37-41.

Lewandowski, K. E., Barrantes-Vidal, N., Nelson-Gray, R. O., Clancy, C., Kepley, H. O. \& Kwapil, T. R. (2006). Anxiety and depression symptoms in psychometrically identified schizotypy. Schizophr Res, 83(2-3), 225-235.

Linney, Y. M., Murray, R. M., Peters, E. R., MacDonald, A. M., Rijsdijk, F. \& Sham, P. C. (2003). A quantitative genetic analysis of schizotypal personality traits. Psychol Med, 33(5), 803-816.

Linney, Y. M. \& Peters, E. R. (2007). The psychological processes underlying symptoms of thought interference in psychosis. Behav Res Ther, 45(11), 2726-2741.

MacLeod, C. \& Hagan, R. (1992). Individual differences in the selective processing of threatening information, and emotional responses to a stressful life event. Behav Res Ther, 30(2), 151-161.

Mata, I., Gilvarry, C. M., Jones, P. B., Lewis, S. W., Murray, R. M. \& Sham, P. C. (2003). Schizotypal personality traits in nonpsychotic relatives are associated with positive symptoms in psychotic probands. Schizophr Bull, 29(2), 273-283.

Merckelbach, H., Muris, P., Nijman, H. \& de Jong, P. J. (1996). Self-reported cognitive failures and neurotic symptomatoloy. Pers Indiv Differ, 20(6), 715-724.

Morrison, A. P. (2001). The interpretation of intrusions in psychosis: An integrative cognitive approach to hallucinations and delusions. Behav and Cogn Psychother, 29(3), 257-276.

Morrison, A. P. \& Baker, C. A. (2000). Intrusive thoughts and auditory hallucinations: a comparative study of intrusions in psychosis. Behav Res Ther, 38(11), 1097-1106.

Morrison, A. P., French, P. \& Wells, A. (2007). Metacognitive beliefs across the continuum of psychosis: comparisons between patients with psychotic disorders, patients at ultra-high risk and non-patients. Behav Res Ther, 45(9), 2241-2246.

Myin-Germeys, I., Delespaul, P. A. \& deVries, M. W. (2000). Schizophrenia patients are more emotionally active than is assumed based on their behavior. Schizophr Bull, 26(4), 847-854.

Myin-Germeys, I., Krabbendam, L., Jolles, J., Delespaul, P. A. \& van Os, J. (2002). Are cognitive impairments associated with sensitivity to stress in schizophrenia? An experience sampling study. Am J Psychiatry, 159(3), 443-449.

Myin-Germeys, I., van Os, J., Schwartz, J. E., Stone, A. A. \& Delespaul, P. A. (2001). Emotional reactivity to daily life stress in psychosis. Arch Gen Psychiatry, 58(12), 1137-1144.

Nuechterlein, K. H., Asarnow, R. F., Subotnik, K. L., Fogelson, D. L., Payne, D. L., Kendler, K. S., et al. (2002). The structure of schizotypy: relationships between neurocognitive and personality disorder features in relatives of schizophrenic patients in the UCLA Family Study. Schizophr Res, 54(1-2), 121-130.

Perala, J., Suvisaari, J., Saarni, S. I., Kuoppasalmi, K., Isometsa, E., Pirkola, S., et al. (2007). Lifetime Prevalence of Psychotic and Bipolar I Disorders in a General Population. Arch Gen Psychiatry, 64(1), 19-28.

Peralta, V. \& Cuesta, M. J. (1999). Dimensional structure of psychotic symptoms: an item-level analysis of SAPS and SANS symptoms in psychotic disorders. Schizophr Res, 38(1), 13-26.

Peralta, V. \& Cuesta, M. J. (2001). How many and which are the psychopathological dimensions in schizophrenia? Issues influencing their ascertainment. Schizophr Res, 49(3), 269-285.

Peralta, V., Cuesta, M. J. \& Farre, C. (1997). Factor structure of symptoms in functional psychoses. Biol Psychiatry, 42(9), 806-815.

Peralta, V., Cuesta, M. J., Martinez-Larrea, A. \& Serrano, J. F. (2001). Patterns of symptoms in neuroleptic-naive patients with schizophrenia and related psychotic disorders before and after treatment. Psychiatry Res, 105(1-2), 97-105.

Ponds, R. W. H. M. (1998). Forgetfulness and cognitive aging. Prevalence, characteristics, and determinants. PhD thesis. Maastricht, The Netherlands: Neuropsych Publishers.

Power, M. J. (1988). Cognitive failures, dysfunctional attitudes, and symptomatology: A longitudinal study. Cog.Emo, 2(2), 133-143.

Pukrop, R., Schultze-Lutter, F., Ruhrmann, S., Brockhaus-Dumke, A., Tendolkar, I., Bechdolf, A., et al. (2006). Neurocognitive functioning in subjects at risk for a first episode of psychosis compared with first- and multiple-episode schizophrenia. J Clin Exp Neuropsychol, 28(8), 1388-1407.

Rabbitt, P. \& Abson, V. (1990). 'Lost and found': some logical and methodological limitations of self-report questionnaires as tools to study cognitive ageing. Br J Psychol, 81(Pt 1), 1-16.

Rossler, W., Salize, H. J., van Os, J. \& Riecher-Rossler, A. (2005). Size of burden of schizophrenia and psychotic disorders. Eur Neuropsychopharmacol, 15(4), 399-409. 
Simons, C. J., Jacobs, N., Jolles, J., van Os, J. \& Krabbendam, L. (2007). Subclinical psychotic experiences and cognitive functioning as a bivariate phenotype for genetic studies in the general population. Schizophr Res, 92(1-3), 24-31.

StataCorp (2006). STATA Statistical Software: Release 9.2. College Station, Texas.

Stefanis, N. C., Hanssen, M., Smirnis, N. K., Avramopoulos, D. A., Evdokimidis, I. K., Stefanis, C. N., et al. (2002). Evidence that three dimensions of psychosis have a distribution in the general population. Psychol Med, 32(2), 347-358.

Taylor, J. L. \& John, C. H. (2004). Attentional and memory bias in persecutory delusions and depression. Psychopathology, 37(5), 233-241.

Toulopoulou, T., Picchioni, M., Rijsdijk, F., Hua-Hall, M., Ettinger, U., Sham, P., et al. (2007). Substantial genetic overlap between neurocognition and schizophrenia: genetic modeling in twin samples. Arch Gen Psychiatry, 64(12), 1348-1355.

van Hooren, S., Versmissen, D., Janssen, I., Myin-Germeys, I., à Campo, J., Mengelers, R., et al. (2008). Social cognition and neurocognition as independent domains in psychosis. Schizophr Res, 103(1-3), 257-265.

van Os, J., Hanssen, M., Bijl, R. V. \& Ravelli, A. (2000). Strauss (1969) revisited: a psychosis continuum in the general population? Schizophr Res, 45(1-2), 11-20.

Vollema, M. G. \& Hoijtink, H. (2000). The multidimensionality of self-report schizotypy in a psychiatric population: an analysis using multidimensional Rasch models. Schizophr Bull, 26(3), 565-575.

Vollema, M. G. \& Ormel, J. (2000). The reliability of the structured interview for schizotypy-revised. Schizophr Bull, 26(3), 619-629.

Vollema, M. G. \& van den Bosch, R. J. (1995). The multidimensionality of schizotypy. Schizophr Bull, 21(1), 19-31.

Wallace, J. C. (2004). Confirmatory factor analysis of the cognitive failures questionnaire: evidence for dimensionality and construct validity. Pers Indiv Differ, 37(2), 307-324.

Wallace, J. C., Kass, S. J. \& Stanny, C. J. (2002). The cognitive failures questionnaire revisited: dimensions and correlates. J Gen Psychol, 129(3), 238-256.

Williams, J. M., Barnhofer, T., Crane, C., Herman, D., Raes, F., Watkins, E., et al. (2007). Autobiographical memory specificity and emotional disorder. Psychol Bull, 133(1), 122-148.

Williams, J. M., Mathews, A. \& MacLeod, C. (1996). The emotional Stroop task and psychopathology. Psychol Bull, $120(1), 3-24$. 

CHAPTER 4

\section{Semantic activation in patients with a psychotic disorder and first-degree relatives}

Stefanie Pfeifer ${ }^{1}$, Niels O. Schiller ${ }^{2,3}$, Jim van Os ${ }^{1,4}$, Wim J. Riedel $^{5}$, Claudia Simons ${ }^{1,6}$ and Lydia Krabbendam ${ }^{1,7}$

\footnotetext{
${ }^{1}$ Department of Psychiatry and Neuropsychology, South Limburg Mental Health Research and Teaching Network, EURON, Maastricht University Medical Centre, Maastricht, The Netherlands

${ }^{2}$ Leiden University Centre for Linguistics (LUCL), Faculty of Humanities, Leiden University, Leiden, The Netherlands

${ }^{3}$ Cognitive Psychology Unit \& Leiden Institute for Brain and Cognition (LIBC), Faculty of Social Sciences, Leiden University, Leiden, The Netherlands

${ }^{4}$ Departiment of Psychosis Studies, Institute of Psychiatry, London SE5 8AF, UK

${ }^{5}$ Department of Neuropsychology and Psychopharmacology, Faculty of Psychology and Neuroscience, Maastricht University, Maastricht, The Netherlands

${ }^{6}$ GGZ Eindhoven, Eindhoven, The Netherlands

${ }^{7}$ Centre Brain and Learning, Department of Psychology and Education, VU University, Amsterdam, The

Netherlands
}

Submitted 


\section{Abstract}

Background. There is evidence that semantic processing deficits are present in patients with psychotic disorder and persons with high risk of psychosis, particularly evident in semantic priming tasks. However, it is less clear whether these deficits are related to abnormal interference or facilitation processes of the semantic activation system.

Methods. Semantic priming and interference effects in speech production were examined within associatively, indirectly and categorically related picture-word pairs using the Picture-Word Interference (PWI) task, with three different stimulus onset asynchronies (SOAs; short, medium, and long) in eighteen patients with a psychotic disorder, twentyfive first-degree relatives of patients with a psychotic disorder, and eighteen healthy controls.

Results. Results showed a stronger interference effect in patients with a psychotic disorder compared to the first-degree relatives group and the healthy control group only at the long SOA. Contradictory to prior reports of increased spreading of activation in patients with a psychotic disorder, in the associative and indirect condition in the present study, patients did not show enhanced semantic priming effects compared to the firstdegree relatives or the controls at any SOA. First-degree relatives did not show effects suggesting similar but attenuated impairments as observed in the patient group. Disorganization symptoms were not associated with interference and priming in any of the groups.

Conclusion. According to this speech production paradigm, an abnormal semantic network is associated with psychotic disorder but does not represent trait familial liability or state disorganization. 


\section{Introduction}

There is accumulating evidence that patients with psychotic disorder display impairments in all aspects of language (DeLisi, 2001). These deficits are particularly prominent in the symptom cluster of disorganized speech, characterized by disturbances in the structure, organization and coherence of speech (Kerns \& Berenbaum, 2002) that may be mediated by abnormalities in the semantic memory network system (Spitzer, 1997).

Semantic memory is assumed to be organized as a network of associated concepts and lexical items (Collins \& Loftus, 1975). These concepts are represented as memory nodes in the semantic network (mental lexicon) and relations between nodes are represented as associative pathways. Activation of a concept node in any part of the memory network spreads through the network to related memory areas (associated nodes). In the speech production process, these nodes - or words - become activated for a short period of time until passive decay or active inhibition occurs (Kiang \& Kutas, 2005). The degree to which a concept activates another one and facilitates its processing is presumably related to the strength of the links between them in the semantic network (Collins \& Loftus, 1975; Leeson, et al., 2005; Spitzer, 1997).

It has been hypothesized that aberrant associations in the semantic network may underlie disorganization symptoms in patients with a psychotic disorder (Spitzer, 1997). Semantic priming procedures form a prominent technique to study the semantic network and the semantic association pathways. Priming refers to the phenomenon that the target stimulus is responded to more quickly when it is preceded by a related prime stimulus (e.g., nest - bird), than when it is preceded by an unrelated prime stimulus (e.g., nest train). In the context of the semantic network model, semantic priming occurs if a prime activates the nearby nodes of a concept in the semantic network, which lowers the threshold of the associated target to become activated. Therefore, the processing of the target, which is already activated to some degree, is facilitated and the target will be recognized faster, compared to unrelated prime target pairs (Collins \& Loftus, 1975; Kiang \& Kutas, 2005; Shelton \& Martin, 1992; Spitzer, et al., 1993). Disorganized speech may be explained by an increased spreading of activation in the semantic network in patients with a psychotic disorder (Maher, 1983), leading to irrelevant intrusions of the activated words into the speech process (Soriano, et al., 2007). 
Several studies found that patients with a psychotic disorder, especially those with formal thought disorder (FTD), show increased semantic priming effects (Manschreck, et al., 1988; Spitzer, et al., 1993). Even greater semantic priming effects in FTD patients have been demonstrated with indirectly related word pairs, in which word pairs are mediated through a non-presented word (i.e., the association between the word pair is only obvious through a mediating associated word (e.g., lemon - (sour) - sweet) (Gouzoulis-Mayfrank, et al., 2003; Moritz, et al., 2001a; Moritz, et al., 2001b; Weisbrod, et al., 1999).

However, conflicting results from previous studies have complicated the interpretation of the semantic priming effect. Several studies reported normal or attenuated priming effects in patients with a psychotic disorder or FTD (Barch, et al., 1996; Goldberg, et al., 1998), rather than increased priming. Methodological issues, for example inadequate stimulus onset asynchrony (SOA) and stimulus duration in these studies, may account for the discrepant findings (for an overview of the methodological problems see (Moritz, et al., 2001b; Moritz, et al., 2002).

The Picture-Word Interference (PWI) task is one paradigm to investigate semantic interference and semantic priming in speech production (Alario, et al., 2000; Schriefers, et al., 1990; Starreveld \& La Heij, 1996). In a typical PWI-task, participants are presented with a to-be-named target picture and an accompanying prime word. It has been shown that whether or not a prime leads to faster processing of the target may critically depend on the nature of the relationship between the two stimuli (Alario, et al., 2000; Cutting \& Ferreira, 1999; Glaser \& Dungelhoff, 1984; La Heij, et al., 1990). That is, priming occurs when two stimuli are associated with each other due to frequent co-occurrence of these items in speech but do not belong to the same semantic category. In contrast, a prime from the same semantic category leads to semantic interference, i.e., slower processing of the target. Lexical competition processes between several activated candidates in the semantic network may explain this phenomenon. If a prime word is activated with its corresponding conceptual representation and a target picture is presented during this activation process, resolving the competition between the simultaneously activated candidates leads to increased reaction times (Alario, et al., 2000; Schriefers, et al., 1990). It can be hypothesized that the abnormal semantic network in psychosis may also lead to aberrant semantic interference effects but this has not been investigated with purely associatively and categorically related stimulus-pairs. 
Previous studies with healthy controls showed that both, the nature of the relationship between prime and target and the SOA, determine whether priming or interference occurs (Alario, et al., 2000; Cutting \& Ferreira, 1999; Glaser \& Dungelhoff, 1984; La Heij, et al., 1990). For example, Alario et al. (2000) found an interference effect for categorically related words (e.g., boat - TRAIN; target picture in capital letters) with short SOA (-114 $\mathrm{ms}$; the prime was presented $114 \mathrm{~ms}$ before the picture) but no effect in the associative condition (e.g., nest - BIRD). The long SOA (-234 ms) yielded a reversed pattern: no effect in the categorical condition but a significant facilitation effect in the associative condition. The explanation behind this phenomenon is likely related to a different representation of the categorical and associative relations among words in the mental lexicon. It is assumed that the semantic (or categorical) interference effect emerges because of competitive processes of simultaneously activated categorically related words, which share similar semantic features. Therefore, at shorter SOAs, due to the simultaneous availability of both candidates, an interference effect occurs, possibly because the selection of the correct candidate (the picture name) requires time to resolve this competition. At longer SOAs, the processing time of the prime is long enough to complete lexical access, and therefore it may not significantly influence the lexical access of the target picture.

On the other hand, the associative facilitation effect at longer SOAs can be explained by a direct associative link between the representations due to frequent co-occurrence of these items in speech production (without sharing semantic similarity). The activation of the prime spreads to its associated units and this activation results in facilitated target processing. One explanation for the lack of this effect at shorter SOAs is that it takes time for the spreading of activation from the prime to the associates including the target (Alario, et al., 2000; Cutting \& Ferreira, 1999; La Heij, et al., 1990; Lupker, 1979).

The aim of the present study was to simultaneously investigate priming and interference effects in speech production in psychosis using the PWI-tasks. An additional advantage of the PWI-task compared to the Lexical Decision Task that previous studies used to investigate semantic priming, is that the PWI-task taps speech production rather than comprehension and is thus more closely related to the observed abnormalities associated with the symptoms of disorganization. Based on the study by Alario and colleagues (2000), the type of the semantic relation between picture-word pairs and SOA was varied. An indirect condition was also included, based on the abnormal priming effects in this condition in patients with a psychotic disorder in previous studies (Gouzoulis-Mayfrank, et 
al., 2003; Spitzer, 1997). There is one study by Soriano and co-workers (2007) that used the PWI-task to investigate semantic memory in FTD and non-FTD patients and found decreased inhibitory automatic processes in patients with FTD, as reflected by fewer errors in the related compared to the unrelated condition (using mainly categorical related picture-word pairs). Soriano et al. (2007) accounted for this lack of effect by unsuccessful attempts of the inhibitory processes to act upon the semantic network. However, the difference to our study was that the prime exposure duration was only 100 $\mathrm{ms}$ in Soriano et al. (2007), presumably precluding deep semantic processing in patients with a psychotic disorder because of slower perceptual processing. Further, the associative facilitation and categorical interference effect was not investigated with increasing SOAs, leaving open the question how priming and interference develop over time in patients with a psychotic disorder.

It was hypothesized that patients will show increased interference in the categorical condition and increased facilitation in the associative and indirect conditions. We expected increased interference in the categorical condition because earlier studies found enlarged interference effects in patients with a psychotic disorder in tasks tapping inhibitory processes (Henik \& Salo, 2004). In addition, increased facilitation was expected in the associative and indirect conditions because patients showed increased semantic and indirect semantic priming effects in earlier studies due to a hyperactive semantic network system (Manschreck, et al., 1988; Moritz, et al., 2001a; Moritz, et al., 2001b; Spitzer, 1993). Finally, it was hypothesized that these abnormalities in the semantic network would be related to disorganization symptom scores (Spitzer, 1997).

Alterations in the semantic network were studied not only in patients with a psychotic disorder but also in individuals at risk for psychosis. Nonaffected first-degree relatives are at higher than average genetic risk for psychotic disorder and share several clinical and cognitive characteristics with patients (Faraone, et al., 1999; O'Connor, et al., 2009), including abnormalities of the semantic network system (Kimble, et al., 2000), although to a lesser extent than is observed in patients. The advantage of this design is that confounding effects occasioned by antipsychotic medication, chronic illness, and cognitive decline can be ruled out (Kimble, et al., 2000). Therefore, if attenuated effects are present in first-degree family members of patients with a psychotic disorder, the aberrant semantic network system may form part of the familial vulnerability to psychosis. 


\section{Methods}

\section{Sample}

The study sample consisted of patients with a diagnosis of nonaffective psychosis, firstdegree relatives (siblings) of patients with nonaffective psychosis, and healthy controls from the general population. Inclusion criteria of the participants of the present study were the following: (i) patients with a history of a nonaffective psychosis according to the DSM-IV and first contact with mental health facilities within the last ten years; (ii) siblings of patients, free of any lifetime nonaffective psychotic disorder; (iii) healthy control participants without the occurrence of any psychotic disorder in either the participant him/herself or any first-degree relatives. Initial selection criteria for all participants were: between 18 and 50 years of age; native speakers of Dutch; an IQ value above 75; normal or corrected to normal vision and no history of dyslexia or neurological disorders such as epilepsy and concussion with loss of consciousness.

Patients were recruited from mental health services and psychiatric hospitals through case loads of selected representative clinicians. All first-degree relatives were sampled through participating patients. Control subjects were recruited through random mailings in nearby municipalities and through advertisements in newspapers. The Comprehensive Assessment of Symptoms and History (CASH; (Andreasen, et al., 1992)) sections on affective and psychotic disorders were used to confirm presence of a diagnosis of nonaffective psychosis in patients, the absence of such a diagnosis in siblings, and absence of a lifetime diagnosis of any psychotic disorder or any current affective disorder in the healthy controls. Additionally, current drug use was assessed with the Composite International Diagnostic Interview (CIDI; (Smeets \& Dingemans, 1993)) and IQ was assessed with the short version of the WAIS III (Blyler, et al., 2000)

The standing ethics committee of Maastricht University Medical Centre approved the study. All participants signed an informed consent conforming to the local ethics committee guidelines. Participants received financial compensation for time and travel associated with the study.

\section{The Picture-Word Interference (PWI) experiment}

Materials. The PWI-task consisted of black and white line drawings of simple objects as target pictures which were paired with the interfering prime words of three different experimental conditions: the associative, categorical and indirect condition. In each 
experimental condition, two types of prime words were selected for each target picture: primes that were semantically related (either associatively, categorically or indirectly) and semantically unrelated control primes.

All primes were Dutch words and chosen from different semantic categories (e.g., animals, vehicles, body parts, food). Pairs of associatively, categorically, indirectly related and unrelated words were selected according to the following criteria:

1. Categorically related picture-word pairs had to pertain to the same semantic category (e.g., animals: wolf - LION) and were not verbally associated.

2. Associatively related picture-word pairs had to be based on a meaning relation of the two stimuli (e.g., glasses - EYE) and were not from the same semantic category.

3. Indirectly related picture-word pairs had to be based on a meaningful relation between the prime and the mediator and between the mediator and the target (e.g., white - (snow) - MOUNTAIN), without belonging to the same semantic category. Further, prime and target of indirectly related pictureword pairs were neither categorically nor associatively related.

4. Unrelated word pairs had neither an associative nor a categorical relation (e.g., car - TABLE), not even indirectly.

Most picture-word pairs of the associative and indirect condition were derived from different Dutch association norms (de Groot \& de Bil, 1987; Lauteslager, et al., 1986; Starreveld \& La Heij, 1996). The association norms of the remaining associative and indirect word pairs were gathered in our laboratory, where 22 healthy subjects other than those participating in the present study were given a sheet with words and instructed to write down, for each word, the word association first coming to their mind. Picture-word pairs of the categorical and the unrelated condition were derived according to the selection criteria previously described. The overall mean association frequency, calculated from the norms, between picture-word pairs in the associative condition was 17 (SD= 11.1), the categorical condition $0.1(S D=0.3)$, and in the indirect condition $0.3(S D=0.8)$. The association frequency between the picture and the mediator was $18(S D=14.7)$ and between the mediator and the prime $22(S D=15.8)$. In the unrelated condition, the associative frequency between picture-word pairs was 0 . 
The prime words had a mean frequency of occurrence of 104.5 per million (SD=190.04, range: $3-899)$ in the associative condition, 37.3 per million $(S D=48.06$, range: $4-191)$ in the categorical condition, and 71.5 per million $(S D=86.83$, range: $4-306$ ) in the indirect condition, as determined by the CELEX data base (Baayen, et al., 1995). Words across conditions were comparable regarding word frequency $(F=15.1, p>0.05)$. Each word was only used once as a prime.

Furthermore, phonological overlap, word length (words were between 2 - 9 letters long), and rhymes were controlled for each word pair. Additionally, word length of each experimental condition was matched with word length of the corresponding unrelated condition.

The drawings of the target pictures were selected from the picture database of the Max Planck Institute (MPI) for Psycholinguistics in Nijmegen, The Netherlands. Only pictures which were not visually similar to each other were selected. Naming agreement (based on a norming study (Schiller \& Koppenhagen, unpublished)) for the pictures used in the present study was $91 \%$.

Design. The three experimental conditions were presented in a block design. In each block, participants were presented with 40 prime target pairs, i.e., 20 from the experimental condition (associatively, categorical, or indirect) and 20 from the unrelated condition. Each target picture was presented twice - once in the experimental condition and once in the unrelated condition. The prime target pairs differed across the experimental conditions but were the same for all three SOAs $(-114,-234$, and $-414 \mathrm{~ms})$. Therefore, each participant saw the same prime target pairs from each condition three times. The order of the three SOAs and the three experimental conditions was counterbalanced for all participants. The prime target pairs in each experiment were pseudo-randomly presented with the restriction that minimally four trials occurred between two similar target pictures. See Table 1 for an overview of the paradigm.

Procedure. Participants were tested individually in a soundproof chamber at Maastricht University. At the beginning of the experiment, participants were familiarized with all pictures. First, the pictures were presented on a personal computer with the corresponding names written next to the picture. After that, only the pictures were presented and participants were asked to name all pictures aloud, to ensure that the 
participants used only the specified name of the picture. Furthermore, the experimenter instructed the participants to avoid using articles (e.g., ' $a$ tiger', or 'the dog') when naming the pictures.

During the experiment, both prime words and target pictures were presented in white at the same position as the fixation cross in the middle of the screen with a viewing distance of approximately $72 \mathrm{~cm}$ against a black background. The pictures appeared with the size of $300 \times 300$ pixels (visual angle $5.55^{\circ} \times 5.55^{\circ}$ ), and the words were presented in lower case letters with font size 30 in Courier new. Each trial consisted of the following sequences of events. First, a fixation cross was presented for $500 \mathrm{~ms}$, followed by a blank screen for 150 $\mathrm{ms}$. Second, the prime word was presented for $220 \mathrm{~ms}$. Third, with the appropriate SOA, the target picture was presented until the participant gave a response or maximally for $3000 \mathrm{~ms}$. The experiment was programmed with the software Presentation (NeurobehaviouralSystems, 2006).

Before the experiment started, participants were presented with ten practice trials to familiarize them with the experimental procedure and to adjust the voice key. These trials were created using the same sources as previously described and not used in the proper experiment. The practice trials were presented in the same order for each participant. The experiment started after a short break in which the participants received feedback about the use of the voice key. The first two trials of each experiment, which were unrelated prime target pairs, not from the experimental stimuli list, were used as warm-up trials and not used in the further analyses. After completion of each block, the participants were given a short break.

All participants were instructed to focus on the fixation cross and to name the picture as fast and accurately as possible, ignoring the previously presented word. Verbal naming latencies (RTs) were registered by a voice key connected to a microphone placed in front of the participants. RTs were measured from the onset of the target picture to the triggering of the voice key when the participant started to give a verbal response. The experimenter registered the occurrence of naming errors and voice key errors.

\section{Assessment of symptomatology}

The disorganized symptom dimension of the Brief Psychiatric Rating Scale (BPRS) version 24 (Lukoff, et al., 1986) was used to determine the severity of disorganized symptomatology over the last two weeks in the patient group. The BPRS is a semi- 
structured interview that consists of 24 items measuring positive/psychotic, negative, disorganized, and depressive symptoms. The disorganized symptom dimension of the BPRS includes nine items (hostility, excitement, mannerisms and posturing, uncooperativeness, self-neglect, bizarre behaviour, elevated mood, motor hyperactivity, distractibility). The severity of symptoms of each item was assessed using a seven-point Likert rating scale, with 1 representing 'absent' and 7 representing 'very severe'. Weighted averages and subsequently standardized scores were calculated for each participant.

Further, the short version of the Schizotypy Personality Questionnaire (SPQ-B; (Dillmann, 2003; Raine \& Benishay, 1995) was used to examine the subclinical disorganized symptom dimension over the last years in first-degree relatives and healthy control participants. The SPQ-B is a self-report questionnaire and consists of 22 items (Raine \& Benishay, 1995) measuring, across an underlying three factor structure, a cognitive-perceptual, an interpersonal, and a disorganized schizotypy dimension. The disorganized dimension includes six items (mannerisms and habits, bizarre person, vague during a conversation, unusual word usage, odd person, unclear communication). Each question was dichotomously assessed (yes/no), and weighted averages and subsequently standardized scores were calculated for each participant.

\section{Statistical analysis}

In order to clean the data from errors, all trials for which the voice key was triggered by noise or did not detect the response of the participant were considered as technical errors and excluded from the analyses. Trials in which participants misnamed the picture, failed to answer or stuttered, were regarded as naming errors and also excluded from further analyses. Percentages of technical errors in the whole dataset were $0.66 \%$ for control participants, $0.63 \%$ for siblings, and $0.83 \%$ for patients, and percentages of naming errors were $0.20 \%, 0.26 \%$, and $0.63 \%$, respectively. No significant differences were found between the three groups on naming error rates $(p>0.05)$. Therefore, further analyses concentrated on reaction time data.

Furthermore, items that produced more than $25 \%$ errors were excluded from the analyses. The item bowl ('schaal') of the associative condition was excluded because it produced a $36 \%$ error rate. In order to normalize the data, reaction times of more than three standard deviations from the mean of each participant were considered as outliers 
and were also excluded from the analyses (1.21\% for control participants, $1.18 \%$ for siblings, and $1.57 \%$ for patients).

For the reaction time (RT) analysis, the prime target pairs were treated as a withinparticipant design with the factor Relatedness (unrelated vs. related) including three levels (associative, categorical or indirect) and a semantic priming/interference effect was computed as follows: mean RTs of an unrelated condition minus the mean RTs of the corresponding related conditions. Therefore, semantic priming effects were expressed as positive values, whereas semantic interference effects were expressed as negative values. All analyses were carried out using STATA version 10.0 (StataCorp, 2007). First, in order to explore whether a difference was significant, matched-paired t-tests were performed for each SOA, each experimental condition and each group separately. In order to investigate group differences of the semantic effects, a one-way analysis of variance (ANOVA) was performed with group as between-subject variable and the semantic effect of each experimental condition as dependent variable, corrected for the a priori specified confounders sex, age, and level of education. Post-hoc multiple comparison analyses (Bonferroni) were performed to test pair wise differences between groups.

Second, linear regression analyses were used to assess the association of the semantic effects and psychotic symptoms (disorganized psychotic dimension of the BPRS in patients and subclinical disorganized symptom dimension of SPQ-B in siblings and control participants) for each experimental condition, SOA, and group, corrected for the a priori demographical confounders. Robust estimates of variance were used, which allow observations that are not independent across groups (i.e., families). Effect sizes were expressed as the standardized regression coefficient (beta).

\section{Results}

\section{Sample}

The sample consisted initially of twenty-one patients with nonaffective psychosis, twentyfive siblings of patients, and twenty healthy control participants. Due to technical difficulties, two participants of the patient group and one participant of the control group were excluded. Additionally, one participant of the control group was excluded because of dyslexia, and one patient was excluded because of incompletion of the task and the BPRS interview. The final study group thus consisted of eighteen patients, twenty-five siblings, 
and eighteen healthy control participants pertaining to 42 different families. All three groups were matched on age, gender, and education. Patients had lower IQ based on the short form of the WAIS III but did not differ on vocabulary as assessed with the Groninger Intelligence Test (GIT). Patients reported drug use more often and had higher levels of symptoms. Socio-demographic and psychopathological characteristics of the sample are given in Table 2.

Based on the CASH interview, seven patients met the criteria for schizophrenia/ paranoid type, four patients met the criteria for schizophrenia/undifferentiated type, three patients met the criteria for schizoaffective disorder, three patients met the criteria for schizophrenia/disorganized type, and two patients met the criteria for psychotic disorder NOS. Four siblings met the criteria for major depressive disorder, single episode and one met the criteria for major depressive disorder, recurrent, in full remission.

At the time of testing, fifteen patients received different second generation antipsychotics (3 amisulpride, 3 olanzapine, 3, risperidone, 2 quetiapine, 2 aripripazole, 1 clozapine, and 1 pimozide) and four patients were taking first generation antipsychotics ( 2 haloperidol, 1 flupentixol, and 1 penfluridol). One patient received 2 different second generation antipsychotics (amisulpride and aripripazole) and one patient received a combination of a first and a second generation antipsychotic (haloperidol and risperidone). Two patients were not currently taking any medication. The mean Chlorpromazine equivalent (CPZ) is given in Table 2.

\section{Semantic priming and interference effects}

Mean reaction times and the semantic effect, listed by SOA $(-114,-234,-414 \mathrm{~ms})$, experimental condition (associative, categorical, and indirect versus the corresponding unrelated condition), and group (control participants, siblings, and patients) are given in Table 3. The obtained difference scores for the semantic effects are shown in Figure 1.

\section{Short SOA (-114 ms).}

Associative condition. Matched-paired t-tests on the mean reaction time data of the associative and the corresponding unrelated condition showed that the $12 \mathrm{~ms}$ priming effect for control participants and the $0.1 \mathrm{~ms}$ difference for patients were not significant ( $p>0.1)$. In siblings, the 17 ms priming effect was significant $(t(24)=-2.9, p=0.008)$. 
Categorical condition. In the categorical condition, an interference effect of $14 \mathrm{~ms}$ in control participants was significant $(t(17)=2.2, p=0.040)$ as well as the $39 \mathrm{~ms}$ interference effect in patients $(t(17)=2.5, p=0.038)$. Also, siblings showed a categorical interference effect of $11 \mathrm{~ms}$, although short of conventional statistical precision $(\mathrm{t}(24)=1.8, \mathrm{p}=0.083)$. Indirect condition. In the indirect condition, the matched-paired t-tests on the mean reaction time data showed that the $3 \mathrm{~ms}$ priming effect for control subjects and the $21 \mathrm{~ms}$ priming effect for patients were not significant ( $p>0.3)$, whereas the $11 \mathrm{~ms}$ priming effect in siblings approached statistical significance $(\mathrm{t}(24)=-1.8, \mathrm{p}=0.083)$.

Group differences of the semantic effect. At the shortest SOA, no significant differences between the three groups in any of the three conditions were found $(p>0.09)$.

\section{Medium SOA (-234 ms).}

Associative condition. The results on the mean reaction time data showed that the $15 \mathrm{~ms}$ priming effect for control subjects $(t(17)=-3.7, p=0.002)$ and the 28 ms priming effect for siblings $(t(24)=-2.7, p=0.013$ ) were significant. For patients, the 21 ms priming effect was not significant $(p>0.2)$.

Categorical condition. In the categorical condition, the $6 \mathrm{~ms}$ priming effect for the control subjects, the $5 \mathrm{~ms}$ interference effect for the siblings, and the $6 \mathrm{~ms}$ priming effect for the patients were not significant ( $p>0.4)$.

Indirect condition. In the indirect condition, the $15 \mathrm{~ms}$ priming effect for control subjects was almost significant $(t(17)=-2.0, p=0.059)$, whereas neither the 6 ms priming effect of the siblings nor the $5 \mathrm{~ms}$ effect of the patients revealed significance $(p>0.3)$.

Group differences of the semantic effect. At medium SOA, there was no significant difference between the three groups in any of the three conditions $(p>0.2)$.

Long SOA (-414 ms).

Associative condition. The matched-paired t-tests on the mean reaction time data showed that the $23 \mathrm{~ms}$ priming effect for control subjects $(t(17)=-2.4, p=0.030)$ and the $29 \mathrm{~ms}$ priming effect for siblings $(t(24)=-3.8, p=0.001)$ were significant. In patients, the $24 \mathrm{~ms}$ priming effect was equally large but not statistically significant ( $p>0.1$ ).

Categorical condition. In the categorical condition, the $40 \mathrm{~ms}$ interference effect for patients was significant $(t(17)=3.3, p=0.004)$, whereas the $10 \mathrm{~ms}$ interference effect in siblings, and the $12 \mathrm{~ms}$ priming effect in control subjects were not significant ( $p>0.1$ ). 
Indirect condition. In the indirect condition, the priming effect of $25 \mathrm{~ms}$ in control participants $(t(17)=-5.5, p=0.000)$ as well as the $16 \mathrm{~ms}$ effect in siblings $(t(24)=-2.6, p=$ $0.016)$, and the $43 \mathrm{~ms}$ effect in patients $(t(17)=-2.6, p=0.02)$ revealed statistical significance.

Group differences of the semantic effect. At this long SOA, a significant group difference was found in the categorical condition $(F(2,58)=6.38, p=0.003)$. Patients showed an increased interference effect compared to controls $(F(1.34)=11.96, p=0.002)$ and siblings $(F(1.41)=5.13, p=0.029)$. Siblings and control participants showed a marginal difference in the categorical condition $(F(1,41)=3.17, p=0.083)$, with an increased interference effect in siblings. In the associative and the indirect condition, no differences between the groups were found $(F(2,58)=0.43, p=0.655)$.

\section{Influence of disorganization symptoms on the semantic effect}

Overall, the associations between the semantic effect and the disorganized symptom dimension, assessed with the BPRS in patients and the SPQ-B in siblings and healthy control participants, showed no meaningful pattern. In patients, no significant association was found ( 6 between -0.1893 and $0.221, p>0.1$ ). In the control and the sibling groups, marginal effects were found. In the control group, a trend towards significant associations was found for the associative condition (short SOA: $B=-0.3879, p=0.060$ ), the indirect condition (medium SOA: $B=-1.0758, p=0.050$ ), and the categorical condition (long SOA: $B=-0.8130, p=0.078)$. In the sibling group, only the associative condition showed a trend towards a significant association with the disorganized dimension at short SOA $(B=-$ $0.3879, p=0.058$ ). However, most of the associations were not in the expected direction of positive associations between priming and the symptom dimension as well as negative associations between interference and the symptom dimension. Therefore, due to a lack of any significant association in the patient group, these trend significant associations are difficult to interpret.

\section{Discussion}

The main purpose of the present study was to examine the categorical interference and associative/indirect facilitation pathways of the semantic network in patients with a 
psychotic disorder, using a PWI-task, which make it possible to investigate priming and interference effects in one paradigm. Additionally, these effects were also investigated in first-degree relatives of patients with a psychotic disorder, in order to investigate whether an abnormal semantic network system could be part of the familial vulnerability to psychosis.

\section{Facilitation and interference in healthy controls}

The validity of the PWI paradigm was supported by our replication of the results by Alario et al. (2000) in the healthy control group. At short SOA, a categorical interference effect was found together with the lack of a significant priming effect in the associative condition. At medium and long SOA, the reversed pattern was observed, showing a facilitation effect in the associative condition and no effect in the categorical condition. This dissociation can be explained by competition processes of categorically related words at short SOAs (Levelt, et al., 1999; Starreveld \& La Heij, 1996) and facilitation processes of associatively related words at long SOAs (Alario, et al., 2000) and further supports the assumption of a different representation of categorical and associative relations among words in the mental lexicon (Alario, et al., 2000; Sailor, et al., 2009). A related conceptualization of different representations of categorical and associative relations among words in the mental lexicon holds that categorical relations produce interference because of competition among lemmas, whereas associates produce facilitation effects because of excitatory connections among word forms (Cutting \& Ferreira, 1999; Sailor, et al., 2009).

The results for the indirect picture-word condition, which was a new element in the current study, resembled the effects described in previous semantic priming studies using word pairs (Moritz, et al., 1999; Spitzer, et al., 1993). At short SOA, healthy control subjects did not show any difference between the indirect and the unrelated condition, however with increasing SOAs the semantic priming effect grew stronger. This pattern of results tentatively suggests that the indirect relations of the picture-word pairs were not immediately available to healthy control subjects but became manifest with longer processing time, possibly supported by a shift from automatic to more controlled strategies with increased SOA (McNamara, 2005; Moritz, et al., 2001b). According to the semantic network model, this increase in priming effects possible emerges due to the 
same facilitative links as in the directly associative relations in the lexical-semantic representation system.

\section{Facilitation and interference in patients with a psychotic disorder}

The main difference between patients and healthy controls was in the categorical condition with long SOA. In line with the initial hypothesis, patients showed a stronger interference effect compared to healthy controls. Specifically, whereas healthy controls did no longer show any interference of the categorically related prime at the long SOA (and even showed a small and non-significant priming effect), patients continued to show evidence of categorical interference at long SOA. Apparently, while in healthy controls the competition between prime and target was resolved at longer SOA, in patients the prime is still competing with the lexical access and selection of the picture name. One explanation for this prolonged interference effect of categorically related items may be that patients with a psychotic disorder may - as a result of alterations in semantic memory - simultaneously activate more (related) categorical items in the semantic network, resulting in increased competition effects which were not resolved with longer SOA. Alternatively, the processing time of the prime of categorical relations of the mental network may take longer, therefore interfering with the lexical access of the picture. However, theories of semantic memory unfortunately make no exact claims about differences between categorical and associative relationships and correlated processing differences. It would be interesting to investigate the time course of this effect at even longer SOAs in future studies. It should be noted, however, that the lack of any interference effect in patients at the medium SOA is at odds with either explanation and calls for caution in interpreting the results.

Difficulties to suppress distractors surrounding a target stimulus (or impaired inhibitory control) is a key feature in psychosis, (Boucart, et al., 1999; Henik \& Salo, 2004). Speculatively, whereas priming effects possibly reflect the bottom-up spreading of activation through the semantic system, the top-down ability to suppress irrelevant distractors is known as inhibitory control. This ability has repeatedly been shown to be compromised in schizophrenia (for example, in terms of increased interference effects on the Stroop Colour-Word Task) (Boucart, et al., 1999; Henik \& Salo, 2004). Therefore, impaired inhibitory control could be an alternative explanation for the categorical interference effect. 
Furthermore, the results support the assumption that the spreading activation process is not an arbitrary process, which would form a disorganization of the semantic system itself (Goldberg, et al., 1998). A completely disorganized semantic network would assume similar effects of all conditions, probably varying in magnitude. This has not been found in the present study, therefore ruling out the possibility of a disorganized semantic network system in patients with a psychotic disorder, assuming a sort of organized structure between relations of concepts in patients with a psychotic disorder.

There were subtle differences between patients and controls in the other conditions, which did not reach statistical significance. Contrary to our hypothesis, patients did not show increased facilitative effects in the associative and indirect conditions. In fact, in patients, facilitative associative effects were absent at short SOA, and comparable to the control group but statistically unreliable at longer SOAs. In the indirect condition, the hypothesized indirect effect was observed at short SOA but it lacked statistical precision in patients, and did not significantly exceed the priming effect seen in the healthy controls. However, even though no significant group differences were found, the facilitative effects at both short and long SOAs were substantially larger in patients compared to controls, supporting a tentative explanation of an increased associative network of indirect relations in patients with a psychotic disorder (Gouzoulis-Mayfrank, et al., 2003; Moritz, et al., 2002; Spitzer, et al., 1993).

\section{Facilitation and interference in individuals at higher than average genetic risk for psychosis}

Contrary to the initial hypothesis, siblings did not show an attenuated version of the categorical interference effect in patients. Overall, siblings did not differ in semantic activation compared to control subjects, suggestive of an intact semantic network system in first degree relatives. This is also contradictory to earlier semantic priming studies showing abnormalities of the semantic network system in schizotypy (Niznikiewicz, et al., 2002) and first degree relatives (Guerra, et al., 2009). Possibly, as suggested by Kimble et al. (2000), deficits in semantic processing (investigated with a N400 sentence processing paradigm) is a psychometric risk indicator expressed as state schizotypy rather than a trait marker indexing familial liability. In the present study, siblings showed similarly low levels of psychopathology as the control group, and a study including first-degree relatives and/or controls with higher levels of (subclinical) psychopathology possibly would have 
yielded different results showing associations with state schizotypy. However, the absence with disorganization symptoms in all three groups suggest that any associations with state schizotypy may be better indexed by other symptoms dimensions.

Notably, there were slight differences between controls and siblings in the associative condition, which did not reach statistical significance in the between-group comparison. Siblings showed a significant priming effect already in the short SOA condition, whereas control subjects only did so at medium SOA. This may possibly point to a slight increase in spreading activation for the associative condition in the sibling group, however this result should be interpreted with caution. Furthermore, this effect was also present in the indirect condition but here the lack of a priming effect in siblings at the medium SOA is unexpected and requires a tentative interpretation of the results. Finally, siblings showed a trend effect in the categorical condition (long SOA) compared to the control group. This trend of categorical interference was in the same direction as the categorical interference effect in the patient group. This may similarly point to a stronger interference effect in siblings than in healthy controls (which was not resolved at the longest SOA) because the prime was still interfering with the lexical access and selection of the target picture name. Again, these results showed only a trend towards significance and should be interpreted with caution. The most conservative conclusion therefore is that on the basis of the current findings, an abnormal semantic network system does not represent trait familial liability to psychotic disorder.

\section{Facilitation and interference in relation to disorganized psychotic symptoms}

Contrary to the initial expectation, there were no large, consistent or meaningful associations between symptom dimensions and either the associative semantic priming or the categorical semantic interference effect. This suggests a relative independence between the abnormalities in the semantic network and symptoms of disorganization. However, one possible reason for the lack of any substantial association is that the level of symptoms of disorganization may have been too low in this sample and thus our experimental method may not have been sensitive enough to detect it (higher levels were found in earlier studies (Barch, et al., 1996; Condray, et al., 1999). Furthermore, in the patient group, the antipsychotic medication and length of illness could be another reason for the decreased influence of psychopathology and the smaller semantic effects. 
However, post-hoc analyses controlling for CPE and duration of illness did not change the pattern of results (results not shown).

\section{Limitations}

The results of the present study must be interpreted in the context of several methodological issues. First, in several previous studies (Goldberg \& Weinberger, 2000; Moritz, et al., 2002; Spitzer, et al., 1993) but not all (Mathalon, et al., 2002), increased semantic priming effects were found mainly in patients with FTD. In the present study, the symptom patterns in the patients did not allow for the formation of an FTD subgroup. Therefore, it may be that analyses using a patient FTD class would have changed the results, suggesting future studies may include an additional FTD subgroup.

Second, because the PWI-paradigm investigates speech production processes and therefore taps different processes in the semantic network compared to Lexical Decision Tasks (which investigate only speech comprehension processes), both tasks were not fully comparable, attributable to the order of the lexical access in speech production and speech comprehension (Cutting \& Ferreira, 1999). However, it can be assumed that the effects of the different conditions were in the same direction, since one previous study showed a decreased priming effect for categorical word pairs in patients with schizophrenia (compared to greater priming effects for associated word pairs), even though this study did not find interference effects (Nestor, et al., 2006). Therefore, it would be important to further investigate associative/indirect and categorical relations with a lexical-semantic priming task with different SOAs.

Third, in the patient group, the medium SOA effect of the categorical and the indirect condition were difficult to interpret. It seems that an inter-stimulus interval of $14 \mathrm{~ms}$ elicited another response mechanism in these groups. Replication of the study with larger sample size and increased SOA intervals could possibly shed more light on this issue.

Fourth, in the sibling group, no difference in semantic activation compared to control subjects was found, indicating an intact semantic network system in first degree family members, which is not in agreement with earlier semantic priming studies (Guerra, et al., 2009). The lack of an effect in siblings may possibly be the result of small sample sizes, not adequate to detect differences using this measure, or an insufficient sensitivity of the PWItask to detect differences in the associative/indirect connection system in the mental lexicon between the groups. 
Fifth, the sample size of the groups was relatively small. However, there are several studies showing significant semantic effects in healthy control groups (Alario, et al., 2000; La Heij, et al., 1990) and in semantic priming studies with patients with psychotic disorder (Soriano, et al., 2007) with comparable sample sizes. However, the results of the present study were not consistently in the expected direction, suggesting that the lack of significant differences were not solely due to lack of power. Still, further studies with larger sample size may usefully provide a clearer picture of the semantic priming effects in psychosis.

In conclusion, careful consideration of the semantic proximity is a necessary condition for establishing more clearly the nature of the mechanism leading to priming and interference effects in speech production which were reported in earlier studies on patients with a psychotic disorder.

\section{Acknowledgments}

This study was supported in part by GROUP (Genetic Risk and Outcome of Psychosis) Maastricht, and funded in part by the European Seventh Framework Programme (EU-GEI consortium). Dr L. Krabbendam was supported by the Netherlands Organization for Scientific Research (VIDI Grant no. 452-07-007). Prof N. O. Schiller was supported by the Netherlands Organization for Scientific Research (VICl Grant no. 453-02-006). We thank all participants for their cooperation. 
Table 1. Overview of the experimental paradigm. The 20 target pictures were paired with 20 prime words from the experimental condition and with 20 prime words from the unrelated condition. These prime word pairs remained the same for all three SOAs.

\begin{tabular}{llll}
\hline SOA & Target picture & Prime word experimental & Prime word unrelated \\
\hline SOA $-114,-234,-414$ & 20 Pictures & 20 associative words & 20 unrelated words \\
SOA $-114,-234,-414$ & 20 Pictures & 20 categorical words & 20 unrelated words \\
SOA $-114,-234,-414$ & 20 Pictures & 20 indirect words & 20 unrelated words \\
\hline
\end{tabular}


Table 2. Demographic and clinical characteristics of the groups.

\begin{tabular}{|c|c|c|c|c|c|}
\hline & $\begin{array}{l}\text { Controls } \\
(\mathrm{N}=18)\end{array}$ & $\begin{array}{l}\text { Siblings } \\
(N=25)\end{array}$ & $\begin{array}{l}\text { Patients } \\
(\mathrm{N}=18)\end{array}$ & $\mathrm{F}^{\mathrm{a}}$ & $\begin{array}{l}\text { Pairwise } \\
\text { comparisons } \\
d\end{array}$ \\
\hline & Mean (SD) & Mean (SD) & Mean (SD) & & \\
\hline Age (years) & $32.5(10.4)$ & $29.4(7.8)$ & $28.6(5.1)$ & $F_{2,49}=1.21, \quad p>0.10$ & \\
\hline $\begin{array}{l}\text { Education } \\
\text { (level achieved) }\end{array}$ & $6.4(1.6)$ & $6.2(1.7)$ & $5.9(1.5)$ & $F_{2,49}=0.35, \quad p>0.10$ & \\
\hline Gender $(m / f)$ & $7 / 11$ & $13 / 12$ & $13 / 5$ & $\mathrm{Chi}^{2, \mathrm{~b}}=4.01, \mathrm{p}>0.10$ & \\
\hline IQ & $117.5(12.4)$ & $111.2(17.8)$ & $97.7(13.9)$ & $F 2,49=7.95, \quad p<0.01$ & $\mathrm{C}>\mathrm{P} ; \mathrm{S}>\mathrm{P}$ \\
\hline GIT (words) & $14(2.03)$ & $13.6(2.2)$ & $14.1(2.6)$ & $F_{2,49}=0.33, \quad p>0.10$ & \\
\hline Drug use \% & $11 \%$ & $32 \%$ & $78 \%$ & $F 2,49=11.87, p<0.01$ & $\mathrm{P}>\mathrm{S}, \mathrm{C}$ \\
\hline $\begin{array}{l}\text { Medication } \\
\text { (CPE mg) }\end{array}$ & - & - & $521(545)$ & Range: 0-2000 & \\
\hline $\begin{array}{l}\text { Length of illness } \\
\text { (years) }\end{array}$ & - & - & $6.28(2.4)$ & Range: $2.9-10$ & \\
\hline \multicolumn{6}{|c|}{ Psychotic symptom scores } \\
\hline \multicolumn{6}{|c|}{ BPRS } \\
\hline positive & $1.06(0.14)$ & $1.02(0.09)$ & $1.84(0.94)$ & $F 2,49=15.02, p<0.01$ & $P>S, C$ \\
\hline negative & $1.00(0.00)$ & $1.03(0.11)$ & $1.54(0.42)$ & $F 2,49=29.74, p<0.01$ & $P>S, C$ \\
\hline depressive & $1.09(0.26)$ & $1.37(0.50)$ & $2.53(1.27)$ & $F_{2,49}=17.57, p<0.01$ & $P>S, C$ \\
\hline disorganized & $1.03(0.10)$ & $1.11(0.13)$ & $1.34(0.38)$ & $F_{2,49}=9.20, \quad p<0.01$ & $\mathrm{P}>\mathrm{S}, \mathrm{C}$ \\
\hline \multicolumn{6}{|l|}{ SPQ-B } \\
\hline disorganized & $0.06(0.13)$ & $0.13(0.25)$ & $0.42(0.3)$ & $F 2,49=11.68, p<0.01$ & $P>S, C$ \\
\hline
\end{tabular}

Notes: GIT, Groninger intelligence test; BPRS, Brief psychiatric rating scale; SPQ-B , Schizotype personality questionnaire.

${ }^{a}$ Group differences were tested by oneway analysis of variance (ANOVA).

${ }^{\mathrm{b}}$ Gender differences between groups were tested by Chi-square analyse test.

${ }^{c}$ Pairwise group differences were tested with Bonferroni pairwise comparisons.

${ }^{d}$ Significant differences between groups at the $p<.05$ level were listed: $C=$ Controls, $S=S i b s, P=P a t i e n t s$. 
Table 3. Mean reaction times (RT in $\mathrm{ms}$; and Standard Deviation (SD)) and mean semantic priming difference (SP) listed for each SOA, each condition, and each group.

\begin{tabular}{|c|c|c|c|c|c|c|c|c|c|}
\hline & $\begin{array}{l}\text { Controls } \\
(\mathrm{N}=18)\end{array}$ & & & $\begin{array}{l}\text { Siblings } \\
(\mathrm{N}=25)\end{array}$ & & & $\begin{array}{l}\text { Patients } \\
(\mathrm{N}=18)\end{array}$ & & \\
\hline & condition & unrelated & & condition & unrelated & & condition & unrelated & \\
\hline & $\mathrm{RT}(\mathrm{SD})$ & $\mathrm{RT}(\mathrm{SD})$ & SP & $\mathrm{RT}(\mathrm{SD})$ & $\mathrm{RT}(\mathrm{SD})$ & SP & $\mathrm{RT}(\mathrm{SD})$ & $\mathrm{RT}(\mathrm{SD})$ & $\mathrm{SP}$ \\
\hline \multicolumn{10}{|c|}{ SOA -114 } \\
\hline ASS & $580(70)$ & $592(72)$ & 12 & $638(128)$ & $650(123)$ & 17 & 754 (169) & 754 (157) & 0.1 \\
\hline CAT & $610(75)$ & $596(77)$ & -14 & $637(87)$ & $626(91)$ & -11 & 796 (163) & 757 (143) & -39 \\
\hline IND & $601(74)$ & $604(62)$ & 3 & 632 (107) & $643(110)$ & 11 & 763 (141) & 784 (164) & 21 \\
\hline \multicolumn{10}{|c|}{ SOA -234 } \\
\hline ASS & $668(80)$ & $683(78)$ & 15 & 704 (107) & 732 (119) & 28 & 809 (122) & $830(145)$ & 21 \\
\hline CAT & $690(82)$ & $696(75)$ & 6 & 721 (106) & 716 (109) & -5 & $827(182)$ & 833 (195) & 6 \\
\hline IND & $683(83)$ & 702 (84) & 15 & 719 (109) & $728(95)$ & 6 & $837(208)$ & 843 (157) & 5 \\
\hline \multicolumn{10}{|c|}{ SOA -414 } \\
\hline ASS & $643(94)$ & $666(92)$ & 23 & $671(121)$ & $699(108)$ & 29 & 799 (182) & $823(171)$ & 24 \\
\hline CAT & $649(72)$ & $660(71)$ & 12 & 701 (106) & $691(97)$ & -10 & 843 (197) & 804 (185) & -40 \\
\hline IND & $642(82)$ & $666(78)$ & 25 & $692(87)$ & 708 (99) & 16 & 812 (185) & 855 (237) & 43 \\
\hline
\end{tabular}

Notes: ASS, Associative condition; CAT, Categorical condition; IND, Indirect condition.

SP-effects were computed as follows: RT baseline condition - RT experimental condition; SOA, Stimulus Onset Asynchrony. 
Figure 1. Associative and indirect priming and categorical interference at Stimulus Onset Asynchronies (SOAs, in ms) of $-114,-234$, and -414 ms for controls, siblings, and patients. Asterisks show significant semantic effects within and between groups at the $0.05 \mathrm{p}$-value.
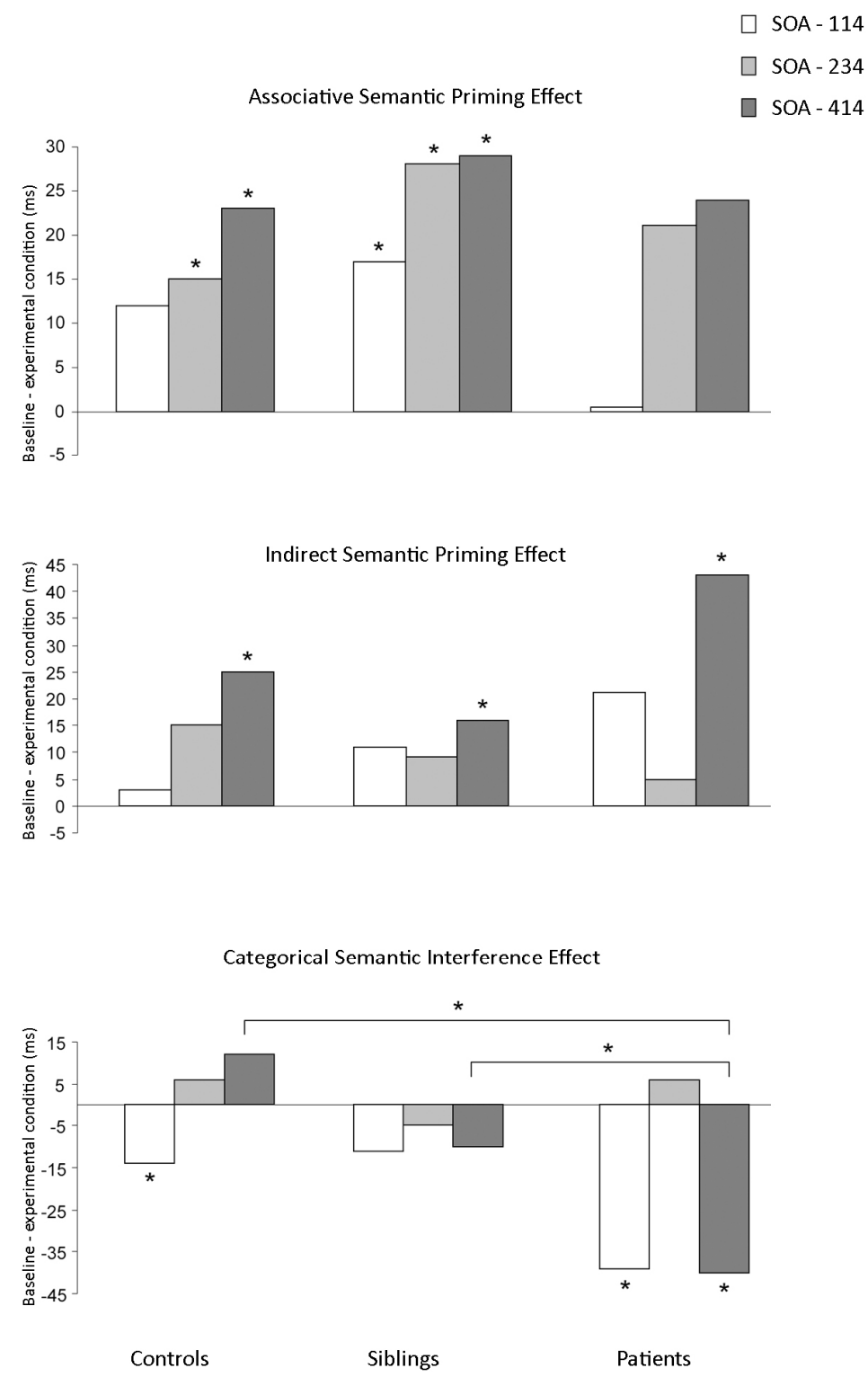


\section{References}

Alario, F. X., Segui, J. \& Ferrand, L. (2000). Semantic and associative priming in picture naming. Q J Exp Psychol A, 53(3), 741-764

Andreasen, N. C., Flaum, M. \& Arndt, S. (1992). The Comprehensive Assessment of Symptoms and History (CASH). An instrument for assessing diagnosis and psychopathology. Arch Gen Psychiatry, 49(8), 615623.

Baayen, R. H., Piepenbrock, R. \& Gulikers, L. (Eds.). (1995). The CELEX lexical database, Linguistic Data Consortium. University of Pennsylvania: Philadelphia

Barch, D. M., Cohen, J. D., Servan-Schreiber, D., Steingard, S., Steinhauer, S. S. \& van Kammen, D. P. (1996). Semantic priming in schizophrenia: an examination of spreading activation using word pronunciation and multiple SOAs. J Abnorm Psychol, 105(4), 592-601.

Blyler, C. R., Gold, J. M., lannone, V. N. \& Buchanan, R. W. (2000). Short form of the WAIS-III for use with patients with schizophrenia. Schizophr Res, 46(2-3), 209-215.

Boucart, M., Mobarek, N., Cuervo, C. \& Danion, J. M. (1999). What is the nature of increased Stroop interference in schizophrenia? Acta Psychol (Amst), 101(1), 3-25.

Collins, A. M. \& Loftus, E. F. (1975). A spreading activation theory of semantic processing. Psychol Rev, 82, $407-$ 428.

Condray, R., Steinhauer, S. R., Cohen, J. D., van Kammen, D. P. \& Kasparek, A. (1999). Modulation of language processing in schizophrenia: effects of context and haloperidol on the event-related potential. Biol Psychiatry, 45(10), 1336-1355.

Cutting, J. C. \& Ferreira, V. S. (1999). Semantic and phonological information flow in the production lexicon. J Exp Psychol Learn Mem Cogn, 25(2), 318-344.

de Groot, A. M. B. \& de Bil, J. M. (1987). Nederlandse Woordassociatienormen Met Reactietijden: 100 Woordassociaties Op 240 Substantieven, 80 Adjectieven En 80 Verba: Swets \& Zeitlinger.

DeLisi, L. E. (2001). Speech disorder in schizophrenia: Review of the literature and exploration of its relation to the uniquely human capacity for language. Schizophr Bull, 27(3), 481-496.

Dillmann, J. (2003). Negative Priming als kognitiver Vulnerabilitätsmarker bei Personen mit Schizotypie auf Basis faktor- und clusteranalytisch ermittelter multidimensionaler Merkmale. Friedrich-Schiller-Universitaet, Jena.

Faraone, S. V., Seidman, L. J., Kremen, W. S., Toomey, R., Pepple, J. R. \& Tsuang, M. T. (1999). Neuropsychological functioning among the nonpsychotic relatives of schizophrenic patients: a 4-year follow-up study. $J$ Abnorm Psychol, 108(1), 176-181.

Glaser, W. R. \& Dungelhoff, F. J. (1984). The time course of picture-word interference. J exp Psychol Human, 10(5), 640-654

Goldberg, T. E., Aloia, M., Gourovitch, M., Missar, D., Pickar, D. \& Weinberger, D. (1998). Cognitive Substrates of Thought Disorder, I: The Semantic System. Am J Psychiatry, 155, 1671-1676.

Goldberg, T. E. \& Weinberger, D. R. (2000). Thought Disorder in Schizophrenia: A Reappraisal of Older Formulations and an Overview of Some Recent Studies. Cognit Neuropsychiatry, 5(1), 1 - 19.

Gouzoulis-Mayfrank, E., Voss, T., Morth, D., Thelen, B., Spitzer, M. \& Meincke, U. (2003). Semantic hyperpriming in thought-disordered patients with schizophrenia: state or trait? A longitudinal investigation. Schizophr Res, 65(2-3), 65-73.

Guerra, S., Ibáñez, A., Martín, M., Bobes, M. A., Reyes, A., Mendoza, R., et al. (2009). N400 deficits from semantic matching of pictures in probands and first-degree relatives from multiplex schizophrenia families. Brain Cogn, 70(2), 221-230.

Henik, A. \& Salo, R. (2004). Schizophrenia and the stroop effect. Behav Cogn Neurosci Rev, 3(1), 42-59.

Kerns, J. G. \& Berenbaum, H. (2002). Cognitive impairments associated with formal thought disorder in people with schizophrenia. J Abnorm Psychol, 111(2), 211-224.

Kiang, M. \& Kutas, M. (2005). Association of schizotypy with semantic processing differences: an event-related brain potential study. Schizophr Res, 77(2-3), 329-342.

Kimble, M., Lyons, M., O'Donnell, B., Nestor, P., Niznikiewicz, M. \& Toomey, R. (2000). The effect of family status and schizotypy on electrophysiologic measures of attention and semantic processing. Biol Psychiatry, 47(5), 402-412

La Heij, W., Dirkx, J. \& Kramer, P. (1990). Categorical interference and asscoiative priming in picture naming. Brit J Psychol, 81, 511-525.

Lauteslager, M., Schaap, T. \& Schievels, D. (1986). Schriftelijke Woordassociatienormen Voor 549 Nederlandse Zelfstandige Naamwoorden: Swets \& Zeitlinger B.V. 
Leeson, V. C., Simpson, A., McKenna, P. J. \& Laws, K. R. (2005). Executive inhibition and semantic association in schizophrenia. Schizophr Res, 74(1), 61-67.

Levelt, W. J., Roelofs, A. \& Meyer, A. S. (1999). A theory of lexical access in speech production. Behav Brain Sci, 22(1), 1-38; discussion 38-75.

Lukoff, D., Nuechterlein, K. H. \& Ventura, J. (1986). Manual for the Expanded Brief Psychiatric Rating Scale. Schizophr Bull, 12, 594-602.

Lupker, S. J. (1979). The semantic nature of response competition in the picture-word interference task. Mem Cognition, 7, 485-495.

Maher, B. A. (1983). A tentative theory of schizophrenic utterance. Prog Exp Pers Res, 12, 1-52.

Manschreck, T. C., Maher, B. A., Milavetz, J. J., Ames, D., Weisstein, C. C. \& Schneyer, M. L. (1988). Semantic priming in thought disordered schizophrenic patients. Schizophr Res, 1(1), 61-66.

Mathalon, D. H., Faustman, W. O. \& Ford, J. M. (2002). N400 and automatic semantic processing abnormalities in patients with schizophrenia. Arch Gen Psychiatry, 5(7), 641-648.

McNamara, T. (2005). Semantic Priming. New York: Psychology Press.

Moritz, S., Andresen, B., Domin, F., Martin, T., Probsthein, E., Kretschmer, G., et al. (1999). Increased automatic spreading activation in healthy subjects with elevated scores in a scale assessing schizophrenic language disturbances. Psychol Med, 29(1), 161-170.

Moritz, S., Mersmann, K., Kloss, M., Jacobsen, D., Andresen, B., Krausz, M., et al. (2001a). Enhanced semantic priming in thought-disordered schizophrenic patients using a word pronunciation task. Schizophr Res, 48(2-3), 301-305.

Moritz, S., Mersmann, K., Kloss, M., Jacobsen, D., Wilke, U., Andresen, B., et al. (2001b). 'Hyper-priming' in thought-disordered schizophrenic patients. Psychol Med, 31(2), 221-229.

Moritz, S., Woodward, T. S., Kuppers, D., Lausen, A. \& Schickel, M. (2002). Increased automatic spreading of activation in thought-disordered schizophrenic patients. Schizophr Res, 59(2-3), 181-186.

Nestor, P. G., Valdman, O., Niznikiewicz, M., Spencer, K., McCarley, R. W. \& Shenton, M. E. (2006). Word priming in schizophrenia: associational and semantic influences. Schizophr Res, 82(2-3), 139-142.

NeurobehaviouralSystems (2006). Presentation. Albany.

Niznikiewicz, M. A., Shenton, M. E., Voglmaier, M., Nestor, P. G., Dickey, C. C., Frumin, M., et al. (2002). Semantic dysfunction in women with schizotypal personality disorder. Am J Psychiatry, 159(10), 1767-1774.

O'Connor, M., Harris, J. M., McIntosh, A. M., Owens, D. G. C., Lawrie, S. M. \& Johnstone, E. C. (2009). Specific cognitive deficits in a group at genetic high risk of schizophrenia. Psych Med, 39(10), 1649-1655.

Raine, A. \& Benishay, D. (1995). The SPQ-B: A brief screening instrument for schizotypal personality disorder. J Pers Disord, 9(4), 346-355.

Sailor, K., Brooks, P. J., Bruening, P. R., Seiger-Gardner, L. \& Guterman, M. (2009). Exploring the time course of semantic interference and associative priming in the picture-word interference task. $Q \mathrm{~J}$ Exp Psychol, 62(4), 789-801.

Schiller, N. O. \& Koppenhagen, H. (unpublished). Name agreement for a set of ambiguous and unambiguous black-and-white drawings in Dutch.

Schriefers, H., Meyer, A. S. \& Levelt, W. J. (1990). Exploring the Time Course of Lexical Access in Language Production: Picture-Word Interference Studies. J Mem Lang, 29, 86-102.

Shelton, J. R. \& Martin, R. C. (1992). How semantic is automatic semantic priming? J Exp Psychol Learn Mem Cogn, 18(6), 1191-1210.

Smeets, R. M. W. \& Dingemans, P. M. A. J. (1993). Composite International Diagnostic Interview (CIDI). Amsterdam/Geneva: World Health Organisation.

Soriano, M. F., Jimenez, J. F., Roman, P. \& Bajo, M. T. (2007). Cognitive Substrates in Semantic Memory of Formal Thought Disorder in Schizophrenia. J Clin Exp Neuropsychol, 1-13.

Spitzer, M. (1993). The psychopathology, neuropsychology, and neurobiology of associative and working memory in schizophrenia. Eur Arch Psychiatry Clin Neurosci, 243, 57-70.

Spitzer, M. (1997). A cognitive neuroscience view of schizophrenic thought disorder. Schizophr Bull, 23(1), 29-50.

Spitzer, M., Braun, U., Hermle, L. \& Maier, S. (1993). Associative Semantic Network Dysfunction in ThoughtDisordered Schizophrenic Patients: Direct Evidence from Indirect Semantic Priming. Biol Psychiatry, 34, 864-877.

Starreveld, P. A. \& La Heij, W. (1996). Time-Course Analysis of Semantic and Orthographic Context effects in Picture Naming. J Exp Psychol, 22(4), 896-918.

StataCorp (2007). STATA Statistical Software: Release 10.0. Texas: College Station.

Weisbrod, M., Kiefer, M., Winkler, S., Maier, S., Hill, H., Roesch-Ely, D., et al. (1999). Electrophysiological correlates of direct versus indirect semantic priming in normal volunteers. Brain Res Cognitive Brain Res, 8(3), 289-298. 

CHAPTER 5

\title{
Electrophysiological correlates of automatic spreading of activation in patients with psychotic disorder and \\ first-degree relatives
}

\author{
Stefanie Pfeifer ${ }^{1}$, Niels 0 . Schiller ${ }^{2,3}$, Jim van $0 s^{1,4}$, Wim J. Riedel ${ }^{5}$, Petra Vlamings ${ }^{6}$, \\ Claudia Simons $^{1,7}$ and Lydia Krabbendam ${ }^{1,8}$
}

\begin{abstract}
${ }^{1}$ Department of Psychiatry and Neuropsychology, South Limburg Mental Health Research and Teaching Network, EURON, Maastricht University Medical Centre, Maastricht, The Netherlands

${ }^{2}$ Leiden University Centre for Linguistics (LUCL), Faculty of Humanities, Leiden University, Leiden, The Netherlands

${ }^{3}$ Cognitive Psychology Unit \& Leiden Institute for Brain and Cognition (LIBC), Faculty of Social Sciences, Leiden University, Leiden, The Netherlands

${ }^{4}$ King's College London, King's Health Partners, Department of Psychosis Studies, Institute of Psychiatry, London SE5 8AF, UK

${ }^{5}$ Department of Neuropsychology and Psychopharmacology, Faculty of Psychology and Neuroscience, Maastricht University, Maastricht, The Netherlands

${ }^{6}$ Department of Neurocognitive Developmental Psychology, Faculty of Psychology and Neuroscience, Maastricht University, Maastricht, The Netherlands

${ }^{7}$ GGZ Eindhoven, Eindhoven, The Netherlands

${ }^{8}$ Centre Brain and Learning, Department of Psychology and Education, VU University, Amsterdam, The Netherlands
\end{abstract}

Submitted 


\section{Abstract}

Background. Semantic network abnormalities in patients with psychotic disorder were examined using associative prime-target relations with two short stimulus asynchronies (SOAs; $-250 \mathrm{~ms}$ and $-500 \mathrm{~ms}$; short and long SOA respectively) to assess the time course of automatic and more controlled processes of semantic priming. To investigate whether an aberrant semantic network system is part of the familial liability for psychosis, healthy siblings of patients with psychotic disorder were additionally examined. The N400 eventrelated brain potential (ERP) was used as a probe of semantic processing.

Method. Twenty-two patients with psychotic disorder, twenty siblings of patients with psychotic disorder and twenty controls participated in a Lexical Decision Task and ERPs were recorded to target words that were associatively, indirectly or not related to their preceding prime word.

Results. Patients showed an increased indirect priming effect of the N400 latency only at short SOA, with increased latency of the indirectly related compared to the unrelated condition, while controls did not show an indirect priming effect. The indirect priming N400 latency effect at short SOA was also found in siblings.

Conclusion. The results support the assumption of a hyperactive semantic network in patients with psychotic disorder, which develops under automatic processes and decreases with more controlled processes. Abnormal spreading of activation may underlie part of the familial liability to psychotic disorder. 


\section{Introduction}

Disturbances of thought and language have long been identified as core features of psychotic disorder (Bleuler, 1950), particularly disorganized thinking, or intrusion of inadequate semantic associational processes (Docherty, et al., 1996). Recent theories have posited that disorganized speech in patients with psychotic disorder result from an abnormal increase of spreading of activation in the semantic memory network (Spitzer, 1997).

Support for this hypothesis comes from behavioural reaction time (RT) studies using semantic priming paradigms (Kumar \& Debruille, 2004; Moritz, et al., 2001b; Spitzer, 1997). Semantic priming refers to the tendency to respond to a target stimulus more quickly if the target is preceded by a semantically related prime compared to an unrelated prime. It has been suggested that related words benefit from facilitation processes in the semantic network through an automatic spread of activation from the activated prime (Collins \& Loftus, 1975). According to this model, unusual associations appear as disorganization symptoms in speech production because the activation of associations in the network is increased as a consequence of a disinhibited semantic network. Abnormally large semantic priming effects (enlarged RT difference between the semantic condition and the unrelated condition) for directly associated words (e.g., glasses - eye) has been found in patients with psychotic disorder and especially in patients with formal thought disorder (FTD)(Chenery, et al., 2004; Moritz, et al., 2001a; Nestor, et al., 2006; Spitzer, et al., 1993; Spitzer, et al., 1994). This activation was found to be additionally increased when indirect associates were used (i.e., prime-target relations which are mediated by one other concept e.g., lion - stripes, mediated by the concept 'tiger'), indicative of excessive and further spreading of activation in patients with psychotic disorder (Moritz, et al., 2001b; Moritz, et al., 2002).

However, not all studies found increased direct and indirect semantic priming effects in patients with psychotic disorder but instead described normal (Barch, et al., 1996; Vinogradov, et al., 1992) or reduced (Ober, et al., 1997) effects. Multiple factors may contribute to the emergence - or lack of the semantic priming effect, including short stimulus asynchrony (SOA) and semantic relationship (Alario, et al., 2000; Moritz, et al., 2001b; Nestor, et al., 2006). Duration of SOA influences whether automatic or strategic processes operate in the semantic priming effect. When the prime is presented less than 
about $500 \mathrm{~ms}$ before the target, the mechanism for priming is assumed to be primarily due to automatic activation of the semantic network. At longer SOA, the automatic processes are complemented by controlled processes, meaning that strategic processes and working memory can influence the outcome (Mathalon, et al., 2010). It has been suggested that so-called hyperpriming effects, i.e., increased priming, in patients with psychotic disorder may develop mainly under automatic processing, whereas the opposite effect - reduced priming - may result from controlled processes leading to deficient activation of semantically related items (Kreher, et al., 2008; Minzenberg, et al., 2002; Moritz, et al., 2001b). Another source of variance in the semantic priming effect is the relationship between word-pairs. It has been hypothesized that associative relations (e.g., nest - bird) are represented differently in the mental lexicon than categorical relations (e.g., boat - train) (Alario, et al., 2000; Cutting \& Ferreira, 1999). One study investigated the effect of associative and categorical relationships in patients with psychotic disorder showing that patients had greater priming effects for associated words than for categorically related words (Nestor, et al., 2006).

Since RT as the final outcome variable is potentially confounded by all processes that occur between the presentation of the stimulus and the behavioural response, scalprecorded ERPs provide a more direct measure of neurophysiological events associated with the semantic dysfunction. One ERP component relevant to language processing is the N400, a negative deflection approximately around $400 \mathrm{~ms}$ after onset of a target word which develops in response to any meaningful stimulus (such as a word). The N400 is sensitive to the expectancy of a word in a given context, with unexpected words producing a larger N400 component than expected words (Kutas \& Hillyard, 1980). N400 amplitude can be used to index the degree to which concepts are activated in the semantic network, as a decreased amplitude is indicative of greater activation of these concepts (Kiang, et al., 2008). In a typical Lexical Decision Task, the N400 amplitude increases (i.e., becomes more negative) with semantic distance (more negative in the unrelated condition compared to the related condition) (Weisbrod, et al., 1999). This N400 effect was also found with indirect associations under automatic timing processes, albeit to a lesser degree compared to associative relations, suggesting the $\mathrm{N} 400$ is sensitive to automatic spreading of activation processes (Kreher, et al., 2006; Weisbrod, et al., 1999). Several ERP studies have investigated the latency of the N400 component. In healthy subjects, the N400 latency was found to resemble behavioural results, with the latest 
N400 latency for the unrelated condition, the shortest N400 latency for the related condition and the indirectly related condition in between (Kiefer, et al., 1998; Weisbrod, et al., 1999). A robust finding of previous $\mathrm{N} 400$ studies in patients with psychotic disorder was an overall increased latency of the N400 component, suggesting abnormal semantic processing (Nestor, et al., 1997) and general slowing of information processing (Kimble, et al., 2000; Kumar \& Debruille, 2004; Nestor, et al., 1997). Spitzer (1997) also reported a decreased $\mathrm{N} 400$ latency for the indirectly related condition compared to the unrelated condition, thus showing indirect priming of the N400 latency in patients with psychotic disorder, while this effect was not seen in the healthy control group (Spitzer, et al., 1997). Results of N400 amplitude are more mixed. Increased N400 semantic priming effects have been reported in patients with psychotic disorder, with abnormally small N400 amplitudes to directly associated words (compared to the unrelated condition) in patients with nonFTD (Mathalon, et al., 2002; Mathalon, et al., 2010), and indirectly related words in patients with FTD (Kreher, et al., 2010; Kreher, et al., 2008) at short SOA, indicative of a broader spreading of activation in the semantic network. In contrast, abnormally reduced N400 semantic priming effects have also been found in patients with psychotic disorder, with larger $\mathrm{N} 400$ amplitudes for the direct and indirect associative conditions (indicating no difference between the associatively/indirectly related and the unrelated N400 amplitude) at short and long SOA (Condray, et al., 2003; Kiang, et al., 2008). The reasons for the discrepancies in previous studies are not fully understood yet. Although similar to the behavioural studies, methodological differences such as SOA, relationship between word pairs or sample characteristics between the studies may play a role (Minzenberg, et al., 2002).

One way to further elucidate the nature of the semantic priming effect in patients with psychotic disorder is the study of non-clinical populations with a higher than average risk for developing the disorder, for example, first-degree relatives of patients with psychotic disorder. The advantage of this approach is that some of the confounding variables that may bias patient research can be avoided, e.g., reduced attention capacity, medication and chronic illness (Kimble, et al., 2000; Morgan, et al., 2006; Moritz, et al., 1999). In atrisk populations, similar but less severe impairments have been found in several areas of language function, including semantic priming (Moritz, et al., 1999) and wordpronunciation tasks (Kerns \& Berenbaum, 2000), and these abnormalities may also be present at the level of the N400 component (Guerra, et al., 2009; Niznikiewicz, et al., 
2004; Niznikiewicz, et al., 1999). The aim of the current study was to extend these findings using a Lexical Decision paradigm with direct and indirect semantic associations in patients with psychotic disorder, first-degree relatives (siblings) and healthy controls. Such a comparison may also shed light on whether the aberrant semantic network system in psychosis forms part of the familial liability to psychotic disorder.

The aim of the study was to further elucidate the nature of the semantic network system in relation to/liability for psychotic disorder, by investigating the $\mathrm{N} 400$ component in a semantic priming paradigm in patients and their nonpsychotic first-degree relatives. The design of the study allowed controlling for the impact of the type of the semantic association between prime and target (by using associative prime-target relations only) and SOA (by tapping only automatic processes of SOA -250 and -500 ms but investigating a possible latency shift of the semantic priming effect with this increase of SOA). We expected that across all participants, the $\mathrm{N} 400$ would be largest (most negative) and latest to the unrelated condition but smallest (most positive) and earliest to the associative condition. The $\mathrm{N} 400$ of the indirect condition was expected to be smaller and earlier compared to the unrelated condition, a pattern consistent with previous findings (Weisbrod, et al., 1999). If psychotic disorder is associated with a hyperactive semantic network, the results should show increased associative N400 priming effects. Since indirectly related word pairs were an additional and stronger indicator of excessive spreading of activation in the semantic network, it was hypothesized that prime words would activate indirectly related targets even more strongly in patients than in the control group. This effect will be strongest at shorter SOA, assuming that an increase in SOA would lead to an increase of controlled processes. If an aberrant semantic network system forms part of the familial liability to psychosis, the results for siblings should resemble those of the patients, although at smaller effect size.

\section{Methods}

\section{Sample}

The study sample consisted of patients with a diagnosis of nonaffective psychosis, firstdegree relatives (siblings) of patients with nonaffective psychosis and controls from the general population. Inclusion criteria of the participants of the present study were the following: (i) patients with a history of a nonaffective psychosis according to the DSM-IV 
and first contact with mental health facilities within the last ten years; (ii) siblings of these patients, free of any lifetime nonaffective psychotic disorder; (iii) healthy control participants without the occurrence of any psychotic disorder in either the participant him/herself or any first-degree relatives. Initial selection criteria for all participants were: between 18 and 50 years of age; native speakers of the Dutch language; an IQ value above 80; normal or corrected to normal vision, no history of dyslexia or neurological disorders such as epilepsy and or concussion with loss of consciousness, no psychotic disorder due to a general medical condition and no alcohol and drug related substance disorder according to the DSM-IV.

Patients were recruited from mental health services and psychiatric hospitals through caseloads of selected representative clinicians. All siblings were sampled through participating patients. Control participants were recruited through random mailings in nearby municipalities and through advertisements in newspapers. The Comprehensive Assessment of Symptoms and History (CASH; (Andreasen, et al., 1992)) sections on affective and psychotic disorders were used to confirm presence of a diagnosis of nonaffective psychosis in patients, the absence of such a diagnosis in siblings, and absence of a lifetime diagnosis of any psychotic disorder or any current affective disorder in the healthy controls. Additionally, current drug use was assessed with the Composite International Diagnostic Interview (CIDI; (Smeets \& Dingemans, 1993)) and IQ was assessed with the short version of the WAIS III (Wechsler, 1997). Participants also completed the wordlist subtest of the Groninger Intelligence Test (GIT) as an estimate of general cognitive ability (Luteijn \& van der Ploeg, 1983).

The standing ethics committee of Maastricht University Medical Centre approved the study. All participants signed an informed consent conforming to the local ethics committee guidelines. Participants received financial compensation for time and travel associated with the study.

\section{Assessment of symptomatology}

The Brief Psychiatric Rating Scale (BPRS) version 24 (Lukoff, et al., 1986) was used to determine the severity of positive, negative, depressive and disorganized symptomatology over the last two weeks. The BPRS is a semi-structured interview that consists of 24 items measuring positive/psychotic, negative, disorganized and depressive symptoms. The severity of symptoms of each item was assessed using a seven-point Likert 
rating scale (with 1 indicating 'absent' and 7 indicating 'very severe'). Weighted averages and subsequently standardized scores were calculated for each participant.

\section{Design and Materials}

The Lexical Decision Task consisted of a prime word followed by a target word in four different experimental conditions: associative, indirect, unrelated, and nonword condition. All stimuli consisted of 258 pairs of letter strings (primes and targets). All primes were real Dutch words. Half of the targets were existing Dutch words, and half of the targets were nonwords conforming to Dutch phonotactics. The 129 prime-target combinations with existing target words consisted of 43 associative pairs, 43 indirectly related pairs, and 43 unrelated pairs. Additionally, another 129 nonword combinations were presented. The experiment consisted of two separate blocks of $-250 \mathrm{~ms}$ and -500 ms SOA (hereafter short and long SOA). The prime-target pairs differed across the experimental conditions but were the same for both SOAs.

The participants received ten additional practice trials to familiarize them with the experimental procedure. These trials were taken from the same source as the other trials (described below) and were not used in the experiment. The practice trials were presented in the same order for each participant.

All prime-target pairs for each SOA were presented in 4 blocks, two consisting of 64 trials and two of 65 trials. Therefore, each participant received eight blocks in total. The blocks of each SOA and the SOAs were counterbalanced across participants and the trials were presented in a random order. Additionally, the first two trials of each block were added as warm-up trials (taken from the same source as the other trials and not used in the experiment). After each block, a short period of rest was provided.

All words were chosen from different semantic categories (e.g., animals, vehicles, body parts, food). Pairs of associative, indirectly related and unrelated words were selected according to the following criteria: Associative word pairs were based on a meaning relation (e.g., glasses - EYE) and were not from the same semantic category (e.g., body parts). Indirectly related word pairs were based on a meaning relation between the prime and the mediator and between the mediator and the target (e.g., white - MOUNTAIN, mediated by 'snow'), without being from the same semantic category. Further, prime and target of indirectly related word pairs were not associatively related. Unrelated word pairs had neither an associative nor an indirect relation (e.g., car - TABLE). 
Most word pairs of the associative and the indirect condition were derived from several Dutch association norms (de Groot \& de Bil, 1987; Lauteslager, et al., 1986; Starreveld \& La Heij, 1996). The associations of the remaining associative and indirect word pairs were gathered in our laboratory, where 23 healthy subjects, other than those participating in the present study, were given a sheet with words and were instructed to write down the word association first coming into their mind for each word. Word pairs of the unrelated condition were derived according to the selection criteria that no association and indirect association were found between prime and target. Phonotactically legal nonwords were pronounceable letter strings (e.g., PONT) that were selected from two nonword databases (Zeelenberg \& Pecher, 2002), based on existing Dutch words with change of at least one letter. The nonwords were matched with the words of the other conditions for word length and identity of first letter.

The prime words of each experimental condition had a mean frequency of occurrence of 63.6 per million ( $S D=131.5$, range: 0.3 - 820) for the associative condition; 36.4 per million ( $S D=45.6$, range: 2 - 190) for the indirect condition and 36.0 per million ( $S D=$ 46.93, range: 0.2 - 178) for the unrelated condition, as determined by the CELEX data base (Baayen, et al., 1995). Prime words across conditions were comparable regarding word frequency $(F=1.4, p>0.05)$. The target words of each experimental condition had a mean frequency of occurrence of 191.6 per million $(S D=729.6$, range: 0.1 - 4534) for the associative condition; 109.4 per million (SD=166.2, range: 1 - 961) for the indirect condition and 126.0 per million (SD= 263.4, range: 0.5 - 1219) for the unrelated condition, as determined by the CELEX data base (Baayen, et al., 1995). Target words across conditions were comparable regarding word frequency $(F=0.5, p>0.05)$.

Furthermore, phonological overlap and rhymes were controlled for each prime-target pair, and word length (word length was between 3 and 10 characters) and number of syllables did not differ between prime-target pairs ( $p>0.05$ ). Additionally, word length of each experimental condition was matched with word length of the nonword condition.

\section{Task procedure}

All participants were tested individually in an electrically shielded, soundproof chamber at Maastricht University. The participants were seated in front of a computer screen on which the stimuli were presented. During the experiment, both prime and target words were presented in white against a black background, at the same position as the fixation 
cross, in the middle of the screen with a viewing distance of approximately $72 \mathrm{~cm}$. The words were presented in lower case letters (font size: 30 points, style: Courier new).

Participants were instructed to rest their arms and hands on the table and were informed not to speak, blink or move their eyes while the words are presented on the screen. Online EEG data recordings were collected with Neuroscan software (Neuroscan version 4.2). In the experiment, participants were presented with the prime word, followed by the target word. The task of the participants was to press a button as quickly and accurately as possible to indicate whether the target is an existing word or a nonword. The participants were instructed to press a right button for a word and a left button for a nonword.

Every trial consisted of the following sequence of stimuli. First, a fixation cross was presented for $500 \mathrm{~ms}$. Second, a blank screen was presented for $200 \mathrm{~ms}$ in order to minimize after effects of the fixation cross. Third, the prime word was presented for 250 ms. Fourth, with the appropriate SOA, the target stimulus was presented until the participant gave a response but for maximum of $2000 \mathrm{~ms}$. After the disappearance of the target stimulus and the next fixation cross, a blank screen was presented for $1500 \mathrm{~ms}$. The experiment was programmed with the software Presentation (NeurobehaviouralSystems, 2006) which also registered RTs of button presses responses, measured from the onset of the target stimulus.

\section{EEG recording procedure and data-analysis}

Participants were asked to sleep normally the night before the experiment and to avoid drugs and alcohol the day before the experiment. Electrophysiological data were continuously collected from the scalp via a 64-channel system, using an EasyCap according to the international $10 \%$ system (equidistant electrodes). The on-line reference electrode was placed at the left mastoid and it was re-referenced off-line to the mean of the activity at the two mastoids. The FC5 electrode was used as ground electrode. Signals were filtered with a $.05-100 \mathrm{~Hz}$ band pass filter and digitized at $500 \mathrm{~Hz}$. A bipolar montage placed on the left upper and lower orbital ridge monitored eye blinks and vertical eye movements. Lateral eye movements were measured with a bipolar montage placed on the right and left external cantus. Electrode impedance for each electrode was kept below 5 $\mathrm{K} \Omega$.

During off-line processing of the continuously collected EEG data, epochs of 1350 ms were obtained, including a $450 \mathrm{~ms}$ pre-stimulus interval for the short SOA experiment and 
epochs of $1600 \mathrm{~ms}$, including a $700 \mathrm{~ms}$ pre-stimulus interval for the long SOA experiment. Those trials contaminated by eye movements or amplifier blocking were removed (Gratton, et al., 1983). After baseline correction (using an interval from - 450 to $-250 \mathrm{~ms}$ before target onset for the short SOA and from -700 to -500 ms before target onset for the long SOA), the signal was filtered with a 1-30 $\mathrm{Hz}$ band pass filter (12dB/oct). Any activation of the scalp or eye monitoring below $-75 \mu \mathrm{V}$ and above $+75 \mu \mathrm{V}$ was considered an artifact and was rejected from further analysis. Furthermore, all trials with incorrect responses were rejected from the dataset. Finally, ERPs were computed for each electrode by averaging the remaining trials for each stimulus condition. ERPs were averaged across groups.

For each condition, N400 latency was defined as the interval between target onset and the largest negative peak from $270-520$ ms post-target onset for both SOA experiments for the control and the sibling group and $240-540 \mathrm{~ms}$ for the patient group. All peaks at the $\mathrm{CZ}$ electrode were confirmed by visual inspection and corrected manually. This adjustment remained in the interval of $270-520$ ms for both SOAs. N400 amplitude was defined as the mean voltage from $270-520$ ms post-stimulus for both SOA experiments for the control and the sibling group and $240-540 \mathrm{~ms}$ for the patient group.

\section{Statistical analysis}

All analyses were carried out using SPSS version 16 (SPSS, 2008). First, RTs and errors were analyzed to evaluate the validity of the paradigm. Analyses of variance (ANOVAs) were conducted to test main effects of condition, group and their interactions at both SOAs separately. This resulted in $3 \times 2$ ANOVA with 3 levels of the between-subject factor Group (controls, siblings, patients) and 2 levels of the within-subject factor Condition (associatively related vs. unrelated; indirectly related vs. unrelated), separate for the RTs and errors.

Second, for the ERP analysis, a series of planned mixed model repeated measures analyses of variance (ANOVAs) were performed for 21 electrodes (FZ, CZ, PZ, F3, F5, C3, C5, P3, P5, P03, T7, CP5, F4, F6, C4, C6, P4, P6, P04, T8, CP6). A priori, we were interested in differences between the three groups in the associative N400 priming effect (associative vs. unrelated) and the indirect N400 priming effect (indirect vs. unrelated) for both SOAs separately (short and long). Therefore, the initial ANOVAs, separately for mean amplitude and peak latency, contained 3 levels of the between-subject factor Group (controls, 
siblings, patients) and 2 levels of the within-subject factor Condition (associative vs. unrelated, indirect vs. unrelated). Any significant Group x Condition interaction was split in separated group comparisons in order to investigate differences in associative and indirect N400 between 2 groups: 2 × 2 ANOVA was conducted with 2 levels of the between-subject factor Group (controls vs. patients; controls vs. siblings) and 2 levels of the within-subject factor Condition (associative vs. unrelated, indirect vs. unrelated). Any significant interaction between Group and Condition was followed by within-group ANOVAs to establish whether the N400 priming effects were significant within each group independently (Kreher, et al., 2008). In each analysis, Electrode was a second withinsubject factor (21 electrodes). To investigate scalp distribution, any significant Condition $\mathrm{x}$ Electrode effect was additionally performed at central (FZ, CZ, PZ), left (F3, F5, C3, C5, P3, $\mathrm{P5}, \mathrm{P03}, \mathrm{T7}, \mathrm{CP} 5)$ and right (F4, F6, C4, C6, P4, P6, P04, T8, CP6) brain areas.

All ANOVAs underwent Greenhouse-Geisser epsilon correction to protect against type 1 error resulting from violations of sphericity (Greenhouse \& Geisser, 1959). Since we tested a priori specified hypotheses, the significance level alpha was set at 0.05 .

In an exploratory analysis, the relationship between psychotic symptoms and N400 latency and amplitude in patients with psychotic disorder was investigated by calculating Pearson's Correlation coefficients for the N400 at CZ (where N400 effects were maximal), for each condition and each SOA, to the positive and disorganized psychotic symptom dimension of the BPRS.

\section{Results}

\section{Sample}

The sample initially consisted of 25 patients with a nonaffective psychosis, 24 siblings of patients and 22 healthy controls. Due to technical difficulties, three participants of the patient group, four of the sibling group and two of the control group were excluded.

The final study group thus consisted of 22 patients, 20 siblings and 20 controls pertaining to 55 different families. All three groups were frequency-matched on age, gender and education. Patients had lower IQ compared to the siblings $(F 1,40=4.7, p<0.05)$ but not compared to the controls ( $p>0.1$ ), and controls and siblings did not differ on IQ either ( $p>$ 0.1). The three groups did not differ on either vocabulary as assessed with the Groninger Intelligence Test (GIT) or drug use in the last year. Patients had higher levels of 
psychopathology. In the control group, all subjects were right-handed. In the sibling group, one subject was left-handed and in the patient group two subjects were left-handed. Socio-demographic and psychopathological characteristics of the sample are given in Table 1.

Eleven patients met the criteria for schizophrenia/paranoid type, two for schizophrenia/undifferentiated type, two for schizoaffective disorder, two for psychotic disorder NOS and two for schizophreniform disorder; one patient met the criteria for schizophrenia/residual type, one for schizophrenia/residual chronic type and one for schizophrenia/disorganized type. Five siblings met the criteria for Major Depressive Disorder, Single Episode, in full remission and two met the criteria for Major Depressive Disorder, recurrent, in partial remission.

At the time of testing, fifteen patients received second-generation antipsychotics (i.e., six patients risperidone, three aripiprazole, two amisulpride, two olanzapine, one quetiapine, and one clozapine) and two patients were taking first generation antipsychotics (i.e., one haloperidol and one pimozide). One patient received two different types of secondgeneration antipsychotics (olanzapine, aripripazole), one patient received a combination of one first and one second-generation antipsychotic (haloperidol and aripripazole) and one patient received one first-generation and two second-generation antipsychotics (clothiapine, risperidone and amisulpride). Two patients were not currently taking any medication. The mean Chlorpromazine equivalent (CPE) is given in Table 1. All siblings were medication-free at the time of testing.

\section{Behavioural data}

In order to normalize the data, RTs of more than three standard deviations from the mean of each participant were considered as outliers and were excluded from the RT analyses (La Heij, et al., 1990). All trials in which participants gave incorrect responses were excluded from the RT analyses. The mean number of trials that were used in the short SOA experiment for the associative, indirect and unrelated condition was: $42.0(S D=0.8), 41.0$ $(S D=1.6)$ and $41.5(S D=1.4)$, respectively for the patients; $42.3(S D=0.9), 41.3(S D=1.2)$ and $41.6(\mathrm{SD}=1.1)$, respectively for the siblings; and $42.5(\mathrm{SD}=0.7), 41.3(\mathrm{SD}=1.7)$ and 41.5 $(S D=1.4)$, respectively for the controls. In the long SOA experiment, the mean number of trials for the associative, indirect and unrelated condition was 41.9 (SD=1.3), 41.1 (SD= 1.3) and $41.7(S D=1.0)$, respectively for the patients; $42.4(S D=0.7), 41.5(S D=1.1)$ and 
$41.6(\mathrm{SD}=1.1)$, respectively for the siblings; and $42.5(\mathrm{SD}=0.8), 41.3(\mathrm{SD}=1.8)$ and 41.3 $(S D=1.6)$, respectively for the controls. For both SOAs, there was no significant difference between the three groups with respect to the number of trials of each condition.

Mean RTs and error rates of each group, condition and each SOA are given in Table 2.

Reaction time analysis. There was no main effect of Group across both condition comparisons (associative vs. unrelated, indirect vs. unrelated) at short and long SOA (all Fs $2,58<2.9$, all ps $>0.05)$. There was a main effect of Condition at short SOA (F1,58=28.12, $p<$ $0.001)$ and long SOA $(F 1,58=16.51, p<0.001)$, with decreased RTs for the associative compared to the unrelated condition (associative priming) across all participants. There was no difference in the RTs of the indirectly related and the unrelated condition across all participants, as indicated by non-significant main effects of Condition at both SOAs (all Fs $1,58<1$, all ps> 0.6). There was no Group by Condition interaction across both conditions and both SOAs (all Fs2,58<2.1, all ps>0.1), suggesting that the three groups did not differ in RTs.

Error analysis. Overall, the rates of correct responses of the three groups showed that participants were attending to the stimuli. There was no main effect of Group across both condition comparisons and at both SOAs (all Fs2,58<1, all ps>0.7). There was a main effect of Condition at short SOA $(F 1,58=16.34, p<0.001)$ and long SOA $(F 1,58=15.53, p<0.001)$, with higher accuracy for the associated condition than for the unrelated condition across all participants. There was no main effect of Condition, indicating no difference in error rate in the indirectly related and the unrelated condition across all participants at both SOAs (all Fs1,58<2.6, all ps> 0.1). There was no Group by Condition interaction at either condition comparison or SOA (all Fs2,58<2.5, all ps>0.09).

\section{Event-related potential analysis}

Grand average ERPs of the associative N400 priming effect and the indirect N400 priming effect are shown in Figure 1 and 2, respectively, for the control, the sibling and the patient group.

N400 amplitude: Associative and indirect N400 priming. There was no significant main effect of Group across all conditions and SOAs (all Fs2,59<2.0, all ps>0.1). Reflected by 
significant main effects of Condition at short SOA (F1,59=21.3, $p<0.001)$ and long SOA $(F 1,59=23.5, p<0.001)$, an associative $N 400$ priming effect across all participants was shown, with more negative amplitudes of the unrelated condition compared to the associative condition. Although the difference in N400 amplitude between the associative and the unrelated condition was broadly distributed over the scalp, the difference was largest central and over the right hemisphere for short SOA (Condition $\mathrm{x}$ Electrode interaction: $\mathrm{F} 20,1180=8.7, \mathrm{p}<0.001$ ), and central for long SOA (Condition $\mathrm{x}$ Electrode interaction: $F 20,1180=4.0, p=0.005)$. This distribution was consistent with previous studies of word reading (Federmeier \& Kutas, 1999; Kiang \& Kutas, 2005; Kiang, et al., 2008).

The main effects of the indirectly related compared to the unrelated condition were not significant at both SOAs (short SOA: F1,59=0.3, p> 0.5; long SOA: F1,59=2.5, p>0.1). There was no Group x Condition interaction in the three groups across both conditions and SOAs (all Fs2,59< 1.2, all ps>0.03).

N400 latency: Associative N400 priming. There was no significant main effect of Group at short $(F 2,59=0.1, p>0.8)$ and long SOA $(F 2,59=0.9, p>0.3)$. Comparisons between the associatively related and unrelated conditions showed a significantly earlier N400 for the associative condition across all participants and both SOAs, indicated by significant main effects of Condition (short SOA: F1,59=26.1, $p<0.001$; long SOA: F1,59=8.6, $p=0.005$ ). The difference between the associative and the unrelated condition was broadly distributed over the scalp for both SOAs (short SOA: Condition x Electrode interaction: F20,1180=0.6, p> 0.4), however, at long SOA it was largest at the right hemisphere (Condition $x$ Electrode interaction: $F 20,1180=2.0, p=0.040)$. There was no Group $x$ Condition interaction of the three groups, indicating that the degree of associative priming of $\mathrm{N} 400$ latency was similar across the three groups (short SOA: F1,59=0.5, p>0.6; long SOA: F1,59=0.1, p>0.9).

\section{N400 latency: Indirect N400 priming.}

$\underline{S O A-250 ~ m s}$ : There was no significant main effect of Group ( $F 2,59=0.04, p>0.9)$. The main effect of Condition was marginally significant $(F 1,59=3.0, p=0.087)$, showing faster $N 400$ latencies of the indirect condition compared to the unrelated condition (indirect priming) broadly distributed over the scalp (Condition x Electrode interaction: F20,1180=1.0, p>0.4). The Group $x$ Condition interaction of the three groups approached significance $(F 2,59=2.7$, $p=0.077)$. The $2 \times 2$ ANOVAs showed a significant Group $x$ Condition interaction in the 
control and patient group $(F 1,40=5.0, p=0.030)$ and a marginally significant interaction in the control and sibling group $(F 1,38=3.8, p=0.059)$. The within-group ANOVA in the control group showed no difference in $\mathrm{N} 400$ latency between the indirect and the unrelated condition $(\mathrm{F} 1,19=1.1, \mathrm{p}>0.3$; mean difference between the unrelated and the indirect condition: $-5.20 \mathrm{~ms})$. In contrast, patients did show an significant increase in indirect priming of the $\mathrm{N} 400$ latency $(F 1,21=4.5, p=0.046)$, with faster $\mathrm{N} 400$ of the indirect compared to the unrelated condition (mean difference: $11.55 \mathrm{~ms}$ ). Siblings did not show a statistical difference between the two conditions $(F 1,19=2.7, p=0.116)$, however, the numerical values showed the same pattern of an indirect priming effect as the patient group (mean difference: $11.15 \mathrm{~ms}$, see Figure 3). There was no Group $\mathrm{x}$ Condition $\mathrm{x}$ Electrode interaction, indicating that the indirect $\mathrm{N} 400$ latency priming effect was broadly distributed over the scalp (controls vs. patients: $F_{20,800}=1.0, p>0.4$, controls vs. siblings $F 20,700=1.0, p>0.4)$.

$\underline{S O A}-500$ ms: There was no significant main effect of Group ( $F 2,59=0.9, p>0.4)$. Comparisons between the indirectly related and unrelated condition showed a significantly earlier $\mathrm{N} 400$ for the indirect condition across all participants, indicated by a significant main effect of Condition ( $F 1,59=5.7, p=0.020)$ and broadly distributed over the scalp (Condition $x$ Electrode interaction: $F 20,1180=0.9, p>0.5$ ). There was no Group $x$ Condition interaction of the three groups $(F 2,59=0.91, p>0.9)$, showing that the degree of indirect priming of the $\mathrm{N} 400$ latency was similar across the three groups

\section{Exploratory correlational analyses of N400 amplitudes and N400 latencies with symptom} dimensions in patients with psychotic disorder

Higher positive psychotic symptom scores were correlated with more negative N400 amplitudes of the indirect condition $(r=-0.46, p=0.030)$ and of the unrelated condition ( $r=$ $-0.58, p=0.004)$ at long SOA. All other correlations of positive and disorganization symptoms and N400 amplitude were not significant ( $r$ 's between -0.25 and $0.28, p>0.05$ in all cases).

In addition, higher positive psychotic symptom scores were correlated with slower N400 latencies for the associative condition at short SOA ( $r=0.44, p=0.042)$. All other correlations of positive and disorganization symptoms on the one hand and N400 latency on the other were not significant ( $r$ 's between -0.33 and $0.37, p>0.05$ for all). 


\section{Discussion}

This study examined associative and indirect semantic priming effects by investigating the amplitude and the latency of the ERP N400 component in patients with psychotic disorder and in siblings of patients with psychotic disorder, to further elucidate semantic network abnormalities in psychosis. In line with the initial hypothesis, all participants showed an associative priming effect of the N400 amplitude and latency, with largest (most negative) and latest N400 to the unrelated condition and smallest (least negative) and earliest N400 to the associative condition. Thus, consistent with earlier studies (Kiang, et al., 2008; Kutas \& Hillyard, 1980; Weisbrod, et al., 1999), our data showed that the N400 is a sensitive index of the structure of the semantic network. Further, the current paradigm elicited priming effects at two different SOAs (short: SOA $-250 \mathrm{~ms}$ and long: SOA $-500 \mathrm{~ms}$ ), making it possible to assess the time course of automatic and more controlled processes of semantic priming.

Under automatic processing at short SOA, patients with psychotic disorder showed indirect priming of the $\mathrm{N} 400$ latency, with decreased latency of the indirect compared to the unrelated condition, while controls did not show an indirect priming effect. This latency priming effect was found only at short SOA, supporting the assumption of a hyperactive semantic network in patients with psychotic disorder developing under automatic processes and decreasing with more controlled processes (Mathalon, et al., 2002; Mathalon, et al., 2010). Contrary to the initial hypothesis, no between-group differences in indirect priming of the N400 amplitude were found.

The increased indirect priming effect of the N400 latency in patients with psychotic disorder is largely consistent with earlier behavioural studies under automatic processing, where patients showed faster RTs to the indirect compared to the unrelated condition, whereas controls did not show increased indirect priming effects (Chenery, et al., 2004; Manschreck, et al., 1988; Moritz, et al., 2002; Spitzer, et al., 1993; Weisbrod, et al., 1998). The results of the RTs of the present study did not show indirect semantic priming differences between groups. This may be due to the relatively small sample size, suggesting that priming effects at behavioural level were primarily observable with larger sample sizes (Spitzer, et al., 1993). The dissociation of the behavioural results and the N400 latency possibly indicates an increased sensitivity of neurophysiological markers to semantic processing abnormalities in patients with psychotic disorders. This may be the 
case because at the behavioural level, RT may be influenced by controlled processes, for example, response selection, inhibition and execution. The N400 is a more direct measure of neural processes, providing an earlier time window of the cognitive processes underlying semantic priming than behavioural measures.

Previous studies have not consistently reported increased priming effects of the N400 latency in patients with psychotic disorder (Kiang, et al., 2008; Kreher, et al., 2008; Mathalon, et al., 2010; Nestor, et al., 1997). However, there is one study of Spitzer and colleagues (1997) which is comparable with our results, reporting similar indirect priming effects of the N400 latency in patients with psychotic disorder, with faster N400 latencies in the indirect condition compared to the unrelated condition in patients but not in the control group (Spitzer, et al., 1997). Together with the results from the current study, this is indicative of an abnormal spreading of activation in the semantic network system in patients with psychotic disorder. We could not replicate an indirect priming N400 latency effect in the control group at short SOA, which was demonstrated earlier (Weisbrod, et al., 1999). Possibly, control subjects process indirectly related targets as unrelated, whereas patients exhibit a further spreading of activation between these prime-target relations. At longer SOA, all groups showed an indirect priming effect of the N400 latency, suggesting that the control and the sibling group had 'caught up' with the patients, in spreading activation to more remote associates (Kreher, et al., 2008).

Increased associative priming of N400 amplitude and latency was not found in patients with psychotic disorder. Therefore, the result of increased N400 latency effect in patients with psychosis might be related to inappropriate timing processes of neural activity within the semantic network which is possibly related to a further spread of activation to associations which are further away in the semantic network, instead of a faster spreading activation which can be elicited with directly associated targets. This is in line with previous studies, supporting hyper-priming for indirect associates in the spreading activation model in psychosis (Kreher, et al., 2008; Moritz, et al., 2001b; Moritz, et al., 2002; Spitzer, 1997; Spitzer, et al., 1993; Spitzer, et al., 1997). However, an indirect priming effect of the $\mathrm{N} 400$ amplitude across all participants and, most notably, an increase in this effect in patients with psychotic disorder was not observed. It is possible that increased indirect priming $\mathrm{N} 400$ amplitude effects were particularly visible in patients with FTD, as was found in earlier studies (Kreher, et al., 2010; Kreher, et al., 2008), whereas 
increased indirect priming N400 latency effects were already noticeable in patients with psychotic disorder (without selecting for FTD).

The increased indirect priming N400 latency effect at short SOA was also observed in the sibling group, although this effect lacked statistical precision in the between-group as well as the within-group comparison, possible due to an insufficient sample size. Indeed, inspection of the data revealed that the direction of indirect priming of the N400 latency in siblings was similar to the patient group and in clear contrast to the control group (Figure 3). A cautious interpretation of this indirect priming N400 latency effect in siblings may be a minor increase of spreading activation representing a familial liability to psychotic disorder. However, this interpretation clearly requires additional empirical support from future studies.

The finding that the $\mathrm{N} 400$ amplitude and latency was correlated with the positive psychotic symptom dimension was somewhat unexpected, since the theoretical background suggests that disorganization symptoms in patients with psychotic disorders result from an abnormal spreading activation process in the semantic memory network (Kreher, et al., 2008; Spitzer, 1997). However, our finding is not isolated, as several previous studies (Kiang, et al., 2007, 2008; Mathalon, et al., 2002; Mathalon, et al., 2010; Nestor, et al., 1997; Niznikiewicz, et al., 1997) did not detect correlations between N400 and disorganized symptoms either.

The results of the present study extend previous findings in several ways. First, the study was designed to control for the impact of the type of the semantic association (by using associative prime-target relations only) and SOA (to assess the time course of automatic to more controlled processes of semantic priming by investigating a possible latency shift of the semantic priming effect with an increase in SOA), thus we can assume that the results were not influenced by these factors. Second, an increased indirect priming N400 latency effect was found in siblings, who have a higher than average risk of developing the disorder. This corroborates the findings in the patients, as the effect in siblings is free of confounding effects of antipsychotic medication and other illness-related factors. Third, an increased indirect priming N400 latency effect was also found in patients with psychotic disorder without selecting only patients with FTD, which was previously found to enlarge and reveal priming effects in patients, both at the behavioural (Chenery, et al., 2004; Moritz, et al., 2001a; Spitzer, et al., 1993) and at the neural level (Kreher, et al., 2008; Weisbrod, et al., 1998). A relatively short length of illness (LOI) in the previous study, not 
exceeding 10 years, could be a possible reason for the enlarged priming effect in patients, as it has been shown that priming effects decrease with length of illness (Maher, et al., 1996). Fifth, it was demonstrated that increased indirect priming effects of the N400 latency in patients can be elicited with a traditional Lexical Decision Task, requiring a behavioural response, and is therefore in contrast with the assumption that increased priming effects in patients can be elicited only with implicit tasks (Kreher, et al., 2010).

\section{Limitations}

Siblings and controls were screened for psychotic and affective disorders and were excluded if they had a lifetime history of psychotic disorder. In the present study, seven of the siblings met criteria for a major depressive disorder (five had a single episode in full remission and two had recurrent episodes in partial remission) that could have biased the results of the sibling group. However, previous studies have observed normal N400 effects in patients with major depressive disorders and dysthymia (Deldin, et al., 2006; lakimova, et al., 2009).

At the time of testing, almost all patients were on antipsychotic medication, which may have influenced the magnitude of the observed differences in N400 latency in the indirect priming condition between controls and patients. However, this effect in siblings was in the same direction (albeit not statistically conclusive), suggesting that antipsychotic medication cannot fully explain the observed between-group difference.

We did not find an association between amplitude or latency of the N400 component and the disorganized dimensions. One possible reason could be that the assessment of symptomatology with the BPRS was not sufficiently precise in picking up all aspects of disorganized thinking in patients with psychosis as more specialized instruments (Andresen \& Moritz, 2000; Moritz, et al., 2002).

The sample size of the groups was relatively small which could have influenced the results. However, several studies showed significant priming effects with comparable sample sizes in healthy controls and in patients with psychotic disorder (Kreher, et al., 2008; Mathalon, et al., 2002), suggesting that a lack of power is less likely to have influenced the results. 


\section{Acknowledgments}

This study was supported in part by GROUP (Genetic Risk and Outcome of Psychosis) Maastricht, and funded in part by the European Seventh Framework Programme (EU-GEI consortium). Dr L. Krabbendam was supported by the Netherlands Organization for Scientific Research (VIDI Grant no. 452-07-007). Prof N. O. Schiller was supported by the Netherlands Organization for Scientific Research (VICI Grant no. 453-02-006). We thank all participants for their cooperation. 
Table 1. Demographic and clinical characteristics of the groups.

\begin{tabular}{|c|c|c|c|c|c|}
\hline & $\begin{array}{c}\text { Controls } \\
(\mathrm{N}=20)\end{array}$ & $\begin{array}{l}\text { Siblings } \\
(\mathrm{N}=20)\end{array}$ & $\begin{array}{c}\text { Patients } \\
(\mathrm{N}=22)\end{array}$ & $\begin{array}{l}\text { Group } \\
\text { comparisons }^{a}\end{array}$ & $\begin{array}{l}\text { Pairwise } \\
\text { comparisons }^{c, d}\end{array}$ \\
\hline & Mean (SD) & Mean (SD) & Mean (SD) & & \\
\hline Age (years) & $32.1(10.7)$ & $32.2(8.1)$ & $29.7(8.3)$ & $F_{2,59}=0.5 ; p>.10$ & \\
\hline $\begin{array}{l}\text { Education } \\
\text { (level achieved) }\end{array}$ & $5.9(1.6)$ & $6.2(1.3)$ & $5.6(1.9)$ & $F 2,59=0.8 ; p>.10$ & \\
\hline Gender (m/f) & $9 / 11$ & $12 / 08$ & $12 / 10$ & $\mathrm{Chi}^{2, \mathrm{~b}}=0.9 ; \mathrm{p}>.10$ & \\
\hline IQ & $108.0(11.2)$ & $109.8(17.6)$ & $99.5(13.4)$ & $F 2,59=3.2 ; p<.05$ & $\mathrm{P}<\mathrm{S}$ \\
\hline GIT (words) & $14.4(1.07)$ & $14(2.0)$ & $13.8(2.2)$ & $F 2,59=0.5 ; p>.10$ & \\
\hline Drug use \% & $5.0 \%$ & $15 \%$ & $4.6 \%$ & $F_{2,59}=0.9 ; p>.10$ & \\
\hline $\begin{array}{l}\text { Medication } \\
\text { (CPE mg) }\end{array}$ & - & - & $521(545)$ & & \\
\hline $\begin{array}{l}\text { Length of illness } \\
\text { (years) }\end{array}$ & - & - & $6.28(2.4)$ & Range: $2.9-10$ & \\
\hline
\end{tabular}

\section{Psychotic symptom scores}

\section{BPRS}

positive $\quad 1.02(0.06) \quad 1.13(0.21) \quad 1.76(1.03) \quad F 2,59=8.6 ; p<.00 \quad P>S, C$

negative $\quad 1.13(0.16) \quad 1.34(0.68) \quad 1.71(0.48) \quad F 2,59=7.6 ; p<.00 \quad P>S, C$

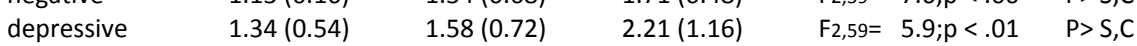

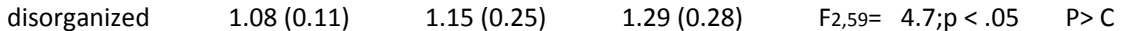

\begin{tabular}{|c|c|}
\hline total & $1.13(0.15)$ \\
\hline
\end{tabular}

Notes: GIT, Groninger Intelligence Test; BPRS, Brief Psychiatric Rating Scale.

${ }^{a}$ Group differences were tested by one-way analysis of variance (ANOVA).

${ }^{b}$ Gender differences between groups were tested by Chi-square analysis.

${ }^{\mathrm{c}}$ Pairwise group differences were tested with Bonferroni pairwise comparisons.

${ }^{d}$ Significant differences between groups at the $\mathrm{p}<.05$ level were listed: $\mathrm{C}=$ Controls, $\mathrm{S}=\mathrm{Sibs}, \mathrm{P}=$ Patients. 
Table 2. Mean reaction times were listed for SOA -250 ms and SOA -500 ms, each condition and each group.

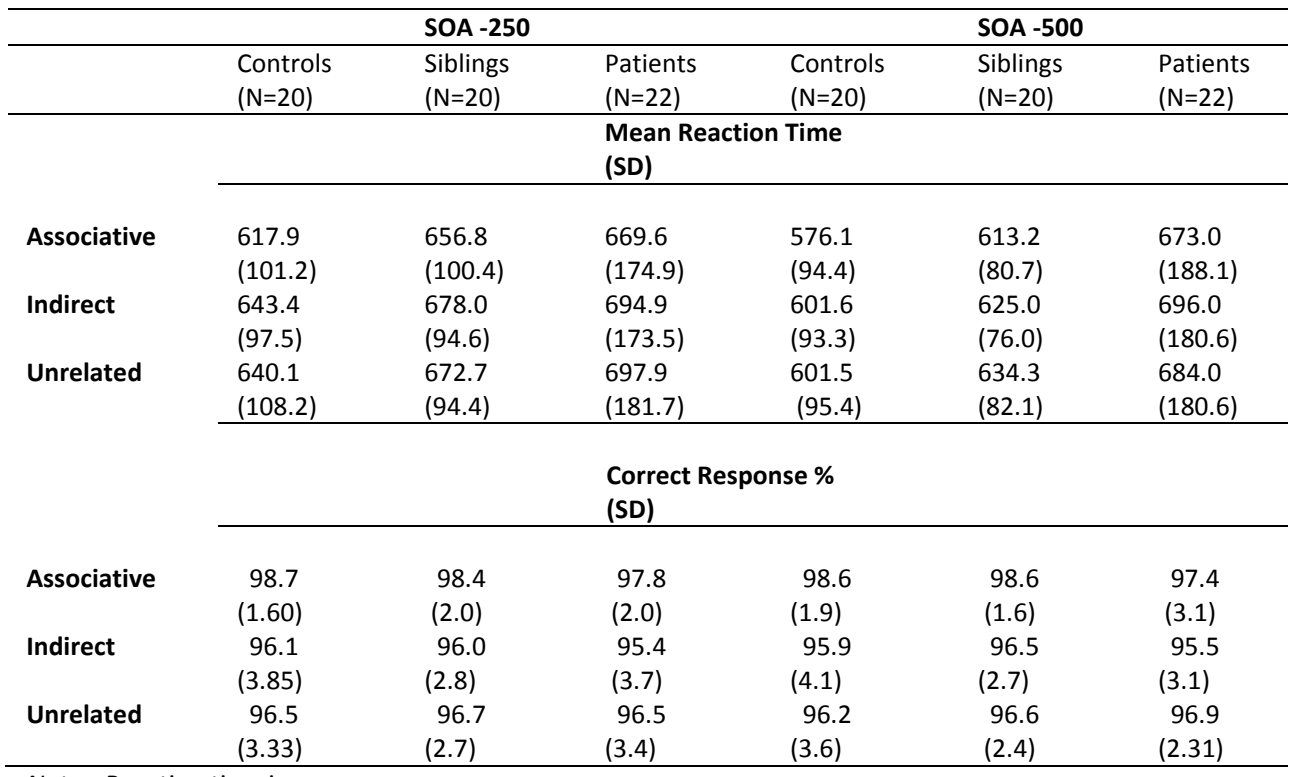

Notes: Reaction time in ms. 
Figure 1. ERPs to the unrelated and associative condition at three electrode sites. Voltage maps show the scalp distribution of the differences in ERPs of the unrelated- associatively related condition.

\section{Associative Priming SOA - 250}

Controls

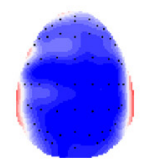

CZ

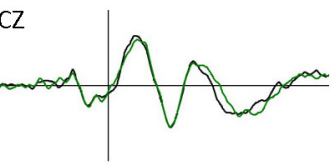

C3

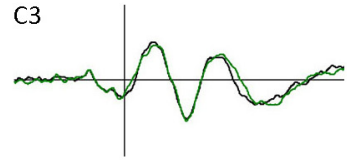

C4

Associative Priming SOA - 500

Controls

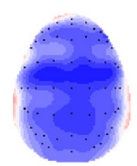

CZ

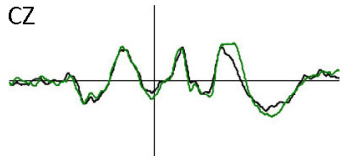

C3

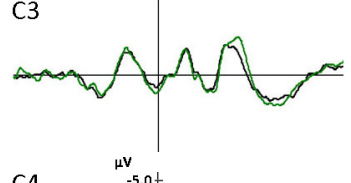

C4

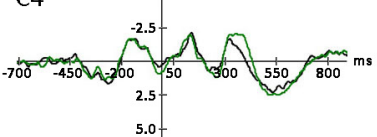

5.0
Siblings
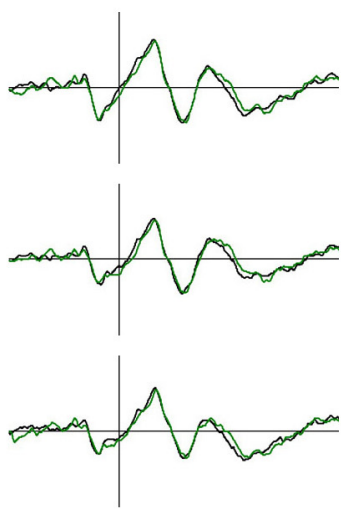

Siblings
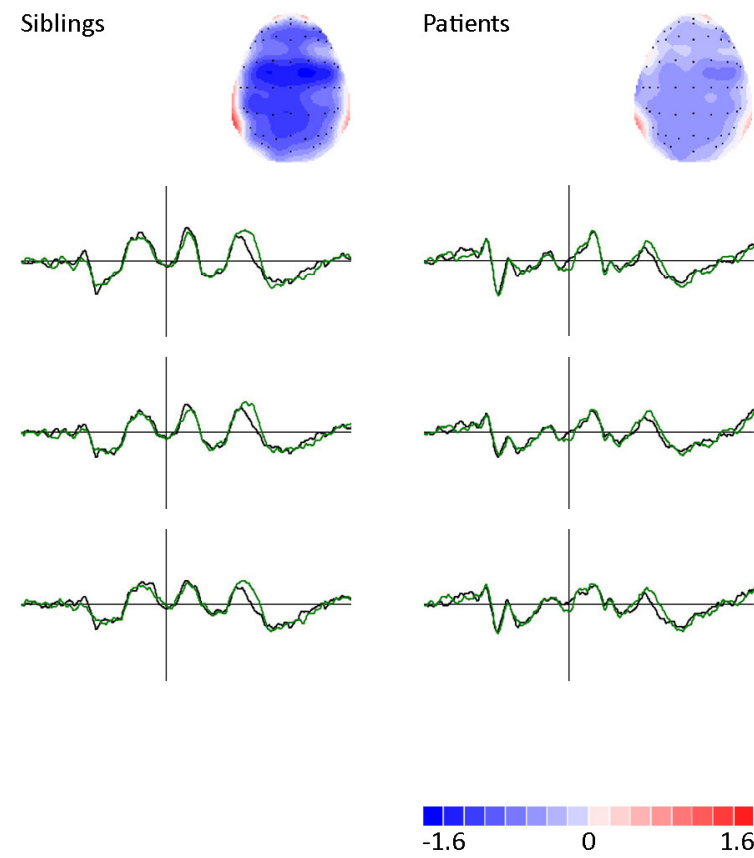

Patients
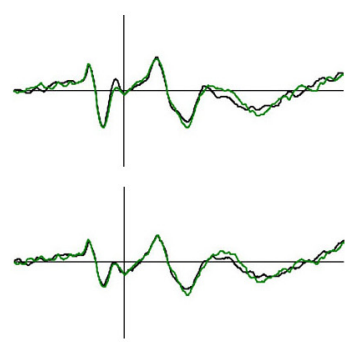

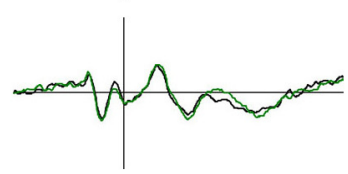

Patients
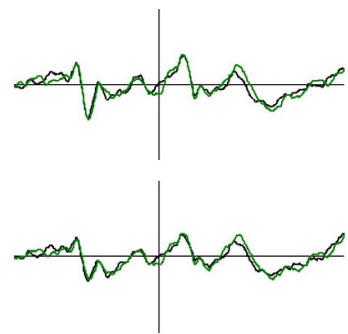

1000010 
Figure 2. ERPs to the unrelated and indirect condition at three electrode sites. Voltage maps show the scalp distribution of the differences in ERPs of the unrelated- indirectly related condition.

\section{Indirect Priming SOA - 250}

Controls

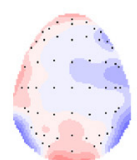

CZ

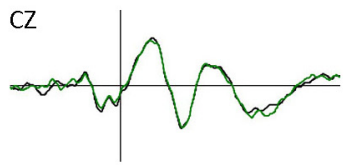

C3
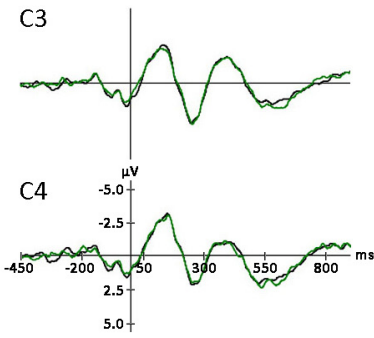

Indirect Priming SOA - 500

Controls
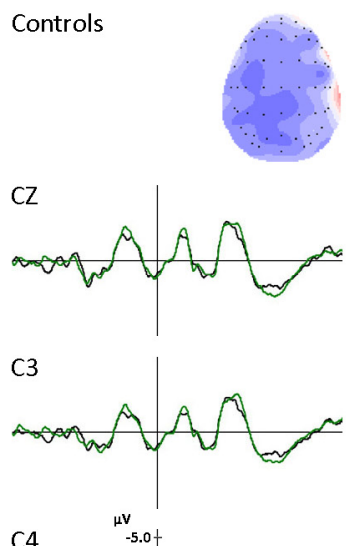

C4 -700
Siblings
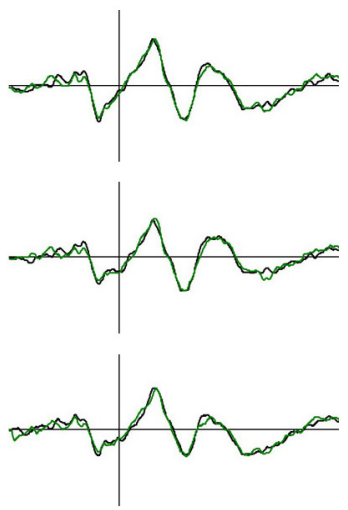

Siblings
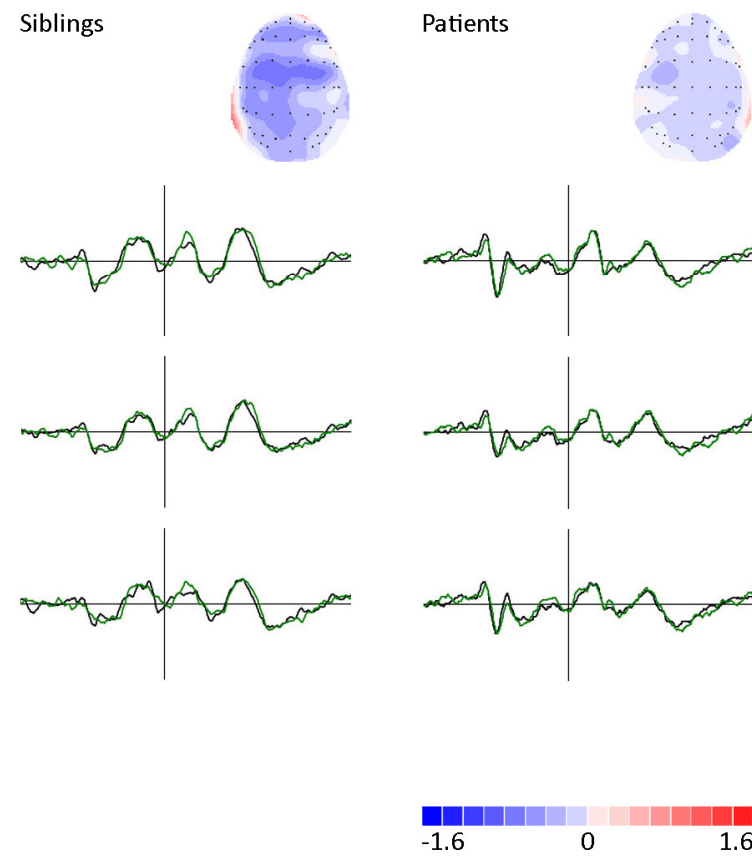

Patients
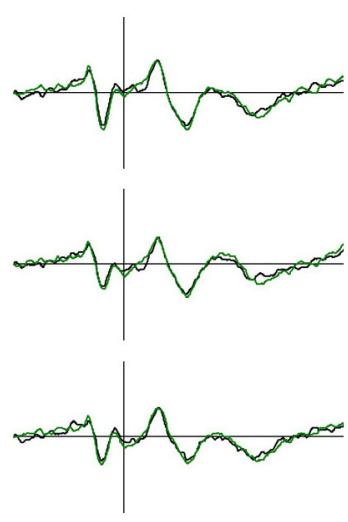

Patients
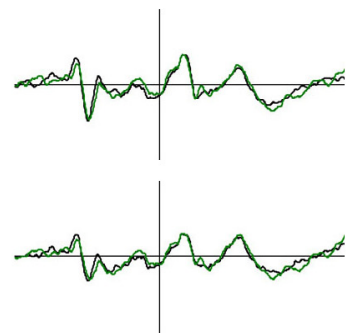

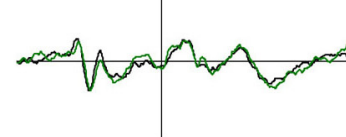


Figure 3. Indirect N400 latency priming effect. Group mean N400 latencies (+standard error of the mean) of the indirect and the unrelated condition for the SOA -250 ms are shown for the control, sibling and the patient group.

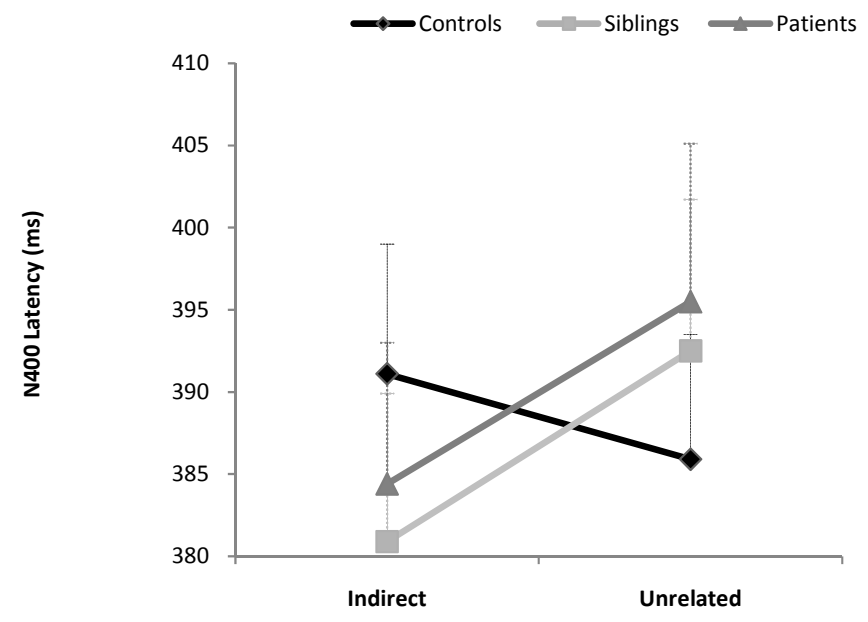




\section{References}

Alario, F. X., Segui, J. \& Ferrand, L. (2000). Semantic and associative priming in picture naming. Q J Exp Psychol A, 53(3), 741-764.

Andreasen, N. C., Flaum, M. \& Arndt, S. (1992). The Comprehensive Assessment of Symptoms and History (CASH). An instrument for assessing diagnosis and psychopathology. Arch Gen Psychiatry, 49(8), 615623.

Andresen, B. \& Moritz, S. (2000). Positive and Negative and Disorganized Symptom Sale (PANADSS). Manual. Westerau, Germany: PPV.

Baayen, R. H., Piepenbrock, R. \& Gulikers, L. (Eds.). (1995). The CELEX lexical database, Linguistic Data Consortium. University of Pennsylvania: Philadelphia

Barch, D. M., Cohen, J. D., Servan-Schreiber, D., Steingard, S., Steinhauer, S. S. \& van Kammen, D. P. (1996). Semantic priming in schizophrenia: an examination of spreading activation using word pronunciation and multiple SOAs. J Abnorm Psychol, 105(4), 592-601.

Bleuler, E. (1950). Dementia praecox or the group of schizophrenias. [Translated by J. Zinkin]. New York: International Universities Press.

Chenery, H. J., Copland, D. A., McGrath, J. \& Savage, G. (2004). Maintaining and updating semantic context in schizophrenia: an investigation of the effects of multiple remote primes. Psychiatry Res, 126(3), 241 252.

Collins, A. M. \& Loftus, E. F. (1975). A spreading activation theory of semantic processing. Psychol Rev, 82, 407428.

Condray, R., Siegle, G. J., Cohen, J. D., van Kammen, D. P. \& Steinhauer, S. R. (2003). Automatic activation of the semantic network in schizophrenia: evidence from event-related brain potentials. Biol Psychiatry, 54(11), 1134-1148.

Cutting, J. C. \& Ferreira, V. S. (1999). Semantic and phonological information flow in the production lexicon. J Exp Psychol Learn Mem Cogn, 25(2), 318-344.

de Groot, A. M. B. \& de Bil, J. M. (1987). Nederlandse Woordassociatienormen Met Reactietijden: 100 Woordassociaties Op 240 Substantieven, 80 Adjectieven En 80 Verba: Swets \& Zeitlinger.

Deldin, P., Keller, J., Casas, B. R., Best, J., Gergen, J. \& Miller, G. A. (2006). Normal N400 in mood disorders. Biol Psychol, 71(1), 74-79.

Docherty, N. M., DeRosa, M. \& Andreasen, N. C. (1996). Communication disturbances in schizophrenia and mania. Arch Gen Psychiatry, 53(4), 358-364.

Federmeier, K. D. \& Kutas, M. (1999). Right words and left words: electrophysiological evidence for hemispheric differences in meaning processing. Brain Res Cogn Brain Res, 8(3), 373-392.

Gratton, G., Coles, M. G. \& Donchin, E. (1983). A new method for off-line removal of ocular artifact. Electroencephalogr Clin Neurophysiol, 55(4), 468-484

Greenhouse, S. W. \& Geisser, S. (1959). On methods in the analysis of profile data. Psychometrika, 24, 95-112.

Guerra, S., Ibáñez, A., Martín, M., Bobes, M. A., Reyes, A., Mendoza, R., et al. (2009). N400 deficits from semantic matching of pictures in probands and first-degree relatives from multiplex schizophrenia families. Brain Cogn, 70(2), 221-230.

lakimova, G., Passerieux, C., Foynard, M., Fiori, N., Besche, C., Laurent, J. P., et al. (2009). Behavioral measures and event-related potentials reveal different aspects of sentence processing and comprehension in patients with major depression. Journal of Affective Disorders, 113(1-2), 188-194.

Kerns, J. G. \& Berenbaum, H. (2000). Aberrant semantic and affective processing in people at risk for psychosis. $J$ Abnorm Psychol, 109(4), 728-732.

Kiang, M. \& Kutas, M. (2005). Association of schizotypy with semantic processing differences: an event-related brain potential study. Schizophr Res, 77(2-3), 329-342.

Kiang, M., Kutas, M., Light, G. A. \& Braff, D. L. (2007). Electrophysiological insights into conceptual disorganization in schizophrenia. Schizophr Res, 92(1-3), 225-236.

Kiang, M., Kutas, M., Light, G. A. \& Braff, D. L. (2008). An Event-Related Brain Potential Study of Direct and Indirect Semantic Priming in Schizophrenia. Am J Psychiatry, 165(1), 74-81.

Kiefer, M., Weisbrod, M., Kern, I., Maier, S. \& Spitzer, M. (1998). Right hemisphere activation during indirect semantic priming: evidence from event-related potentials. Brain Lang, 64(3), 377-408.

Kimble, M., Lyons, M., O'Donnell, B., Nestor, P., Niznikiewicz, M. \& Toomey, R. (2000). The effect of family status and schizotypy on electrophysiologic measures of attention and semantic processing. Biol Psychiatry, $47(5), 402-412$ 
Kreher, D. A., Goff, D. \& Kuperberg, G. R. (2010). Why all the confusion? Experimental task explains discrepant semantic priming effects in schizophrenia under "automatic" conditions: Evidence from Event-Related Potentials. Schizophr Res, 111(1-3), 147-181.

Kreher, D. A., Holcomb, P. J., Goff, D. \& Kuperberg, G. R. (2008). Neural Evidence for Faster and Further Automatic Spreading Activation in Schizophrenic Thought Disorder. Schizophr Bull, 34(3), 473-482.

Kreher, D. A., Holcomb, P. J. \& Kuperberg, G. R. (2006). An electrophysiological investigation of indirect semantic priming. Psychophysiology, 43(6), 550-563.

Kumar, N. \& Debruille, J. B. (2004). Semantics and N400: insights for schizophrenia. J Psychiatry Neurosci, 29(2), 89-98.

Kutas, M. \& Hillyard, S. A. (1980). Reading senseless sentences: brain potentials reflect semantic incongruity. Science, 207(4427), 203-205.

La Heij, W., Dirkx, J. \& Kramer, P. (1990). Categorical interference and asscoiative priming in picture naming. Brit J Psychol, 81, 511-525.

Lauteslager, M., Schaap, T. \& Schievels, D. (1986). Schriftelijke Woordassociatienormen Voor 549 Nederlandse Zelfstandige Naamwoorden: Swets \& Zeitlinger B.V.

Lukoff, D., Nuechterlein, K. H. \& Ventura, J. (1986). Manual for the Expanded Brief Psychiatric Rating Scale. Schizophr Bull, 12, 594-602.

Luteijn, F. \& van der Ploeg, F. (1983). Handleiding Groninger Intelligentietest (GIT) [Manual Groningen Intelligence Test]. Lisse, The Netherlands: Swets \& Zeitlinger.

Maher, B. A., Manschreck, T. C., Redmond, D. \& Beaudette, S. (1996). Length of illness and the gradient from positive to negative semantic priming in schizophrenic patients. Schizophr Res, 22(2), 127-132.

Manschreck, T. C., Maher, B. A., Milavetz, J. J., Ames, D., Weisstein, C. C. \& Schneyer, M. L. (1988). Semantic priming in thought disordered schizophrenic patients. Schizophr Res, 1(1), 61-66.

Mathalon, D. H., Faustman, W. O. \& Ford, J. M. (2002). N400 and automatic semantic processing abnormalities in patients with schizophrenia. Arch Gen Psychiatry, 5(7), 641-648.

Mathalon, D. H., Roach, B. J. \& Ford, J. M. (2010). Automatic semantic priming abnormalities in schizophrenia. Int J Psychophysiol, 75(2), 157-166.

Minzenberg, M. J., Ober, B. A. \& Vinogradov, S. (2002). Semantic priming in schizophrenia: a review and synthesis. J Int Neuropsychol Soc, 8(5), 699-720.

Morgan, C., Bedford, N. \& Rossell, S. L. (2006). Evidence of semantic disorganisation using semantic priming in individuals with high schizotypy. Schizophr Res, 84(2-3), 272-280.

Moritz, S., Andresen, B., Domin, F., Martin, T., Probsthein, E., Kretschmer, G., et al. (1999). Increased automatic spreading activation in healthy subjects with elevated scores in a scale assessing schizophrenic language disturbances. Psychol Med, 29(1), 161-170.

Moritz, S., Mersmann, K., Kloss, M., Jacobsen, D., Andresen, B., Krausz, M., et al. (2001a). Enhanced semantic priming in thought-disordered schizophrenic patients using a word pronunciation task. Schizophr Res, 48(2-3), 301-305

Moritz, S., Mersmann, K., Kloss, M., Jacobsen, D., Wilke, U., Andresen, B., et al. (2001b). 'Hyper-priming' in thought-disordered schizophrenic patients. Psychol Med, 31(2), 221-229.

Moritz, S., Woodward, T. S., Kuppers, D., Lausen, A. \& Schickel, M. (2002). Increased automatic spreading of activation in thought-disordered schizophrenic patients. Schizophr Res, 59(2-3), 181-186.

Nestor, P. G., Kimble, M. O., O'Donnell, B. F., Smith, L., Niznikiewicz, M., Shenton, M. E., et al. (1997). Aberrant semantic activation in schizophrenia: a neurophysiological study. Am J Psychiatry, 154. (5), 640-646.

Nestor, P. G., Valdman, O., Niznikiewicz, M., Spencer, K., McCarley, R. W. \& Shenton, M. E. (2006). Word priming in schizophrenia: associational and semantic influences. Schizophr Res, 82(2-3), 139-142.

NeurobehaviouralSystems (2006). Presentation. Albany.

Niznikiewicz, M. A., Friedman, M., Shenton, M. E., Voglmaier, M., Nestor, P. G., Frumin, M., et al. (2004). Processing sentence context in women with schizotypal personality disorder: An ERP study. Psychophysiology, 41(3), 367-371.

Niznikiewicz, M. A., O'Donnell, B. F., Nestor, P. G., Smith, L., Law, S., Karapelou, M., et al. (1997). ERP assessment of visual and auditory language processing in schizophrenia. J Abnorm Psychol, 106(1), 85-94.

Niznikiewicz, M. A., Voglmaier, M., Shenton, M. E., Seidman, L. J., Dickey, C. C., Rhoads, R., et al. (1999). Electrophysiological correlates of language processing in schizotypal personality disorder. Am J Psychiatry, 156(7), 1052-1058.

Ober, B. A., Vinogradov, S. \& Shenaut, G. K. (1997). Automatic versus controlled semantic priming in schizophrenia. Neuropsychology, 11(4), 506-513.

Smeets, R. M. W. \& Dingemans, P. M. A. J. (1993). Composite International Diagnostic Interview (CIDI). Amsterdam/Geneva: World Health Organisation. 
Spitzer, M. (1997). A cognitive neuroscience view of schizophrenic thought disorder. Schizophr Bull, 23(1), 29-50.

Spitzer, M., Braun, U., Hermle, L. \& Maier, S. (1993). Associative Semantic Network Dysfunction in ThoughtDisordered Schizophrenic Patients: Direct Evidence from Indirect Semantic Priming. Biol Psychiatry, 34, 864-877.

Spitzer, M., Weisbrod, M., Winkler, S. \& Maier, S. (1997). Event-related potentials in semantic speech processing by schizophrenic patients. Nervenarzt, 68(3), 212-225.

Spitzer, M., Weisker, I., Winter, M., Maier, S., Hermle, L. \& Maher, B. A. (1994). Semantic and phonological priming in schizophrenia. J Abnorm Psychol, 103(3), 485-494.

SPSS (2008). SPSS for Windows Rel. 16.0.2. Chicago: SPSS Inc.

Starreveld, P. A. \& La Heij, W. (1996). Time-Course Analysis of Semantic and Orthographic Context effects in Picture Naming. J Exp Psychol, 22(4), 896-918.

Vinogradov, S., Ober, B. A. \& Shenaut, G. K. (1992). Semantic priming of word pronunciation and lexical decision in schizophrenia. Schizophr Res, 8(2), 171-181.

Wechsler, D. (1997). Wechsler Adult Intelligence Scale- 3rd Edition. San Antonio, TX: Harcourt Assessment.

Weisbrod, M., Kiefer, M., Winkler, S., Maier, S., Hill, H., Roesch-Ely, D., et al. (1999). Electrophysiological correlates of direct versus indirect semantic priming in normal volunteers. Brain Res Cognitive Brain Res, 8(3), 289-298.

Weisbrod, M., Maier, S., Harig, S., Himmelsbach, U. \& Spitzer, M. (1998). Lateralised semantic and indirect semantic priming effects in people with schizophrenia. Br J Psychiatry, 172, 142-146.

Zeelenberg, R. \& Pecher, D. (2002). False memories and lexical decision: even twelve primes do not cause longterm semantic priming. Acta Psychol (Amst), 109(3), 269-284. 

CHAPTER 6

Epilogue 


\section{Epilogue}

Impaired cognitive functioning has widely been recognised as a core feature in patients with psychotic disorder (Heinrichs \& Zakzanis, 1998; Keefe, et al., 2006; Krabbendam, et al., 2001). However, subtle cognitive abnormalities in similar domains have also been found to covary with the subclinical psychosis phenotype, since cognitive abnormalities were found in nonaffected first-degree relatives of patients with psychotic disorder (Hughes, et al., 2005; Krabbendam, et al., 2001; Kremen, et al., 1994; Sitskoorn, et al., 2004) and in individuals with psychometrically defined risk for the disorder (Dinn, et al., 2002; Krabbendam, et al., 2005b; Simons, et al., 2007; VogImaier, et al., 1997). This finding suggests that cognitive impairments are promising markers of psychosis liability and can be considered as candidate intermediate phenotypes of psychosis. Intermediate phenotypes are heritable traits underlying the disorder with a simpler genetic basis than the more complex psychosis phenotype (van Os \& Kapur, 2009), thus making them good candidates for detecting underlying mechanisms that play a part in psychotic symptom formation (see Figure 1).

According to the criteria of Gottesman and Gould (2003), cognition can form a useful intermediate phenotype because cognitive deficits are (i) heritable, (ii) present in relatives of patients with psychotic disorder and in individuals from the general population with subclinical psychotic symptoms, (iii) cluster in families of patients with psychotic disorder, and (iv) are associated with psychotic disorder but are not the consequence of the illness (Gottesman \& Gould, 2003).

The studies described in this thesis focused on cognition as intermediate phenotype, how cognitive intermediate phenotypes are related to symptom formation in psychosis and how cognition as intermediate phenotype interacts with environmental influences. More specifically, it was examined whether the presence of various cognitive intermediate phenotypes is associated with specific symptoms of psychosis and how environmental risk factors interact with cognitive intermediate phenotypes in the development of specific symptoms of psychosis. The broader psychosis phenotype was the focus of the studies described in chapters 2 and 3 , examining general cognitive impairments in individuals with a psychometrically defined risk for psychosis. In chapters 4 and 5, specific cognitive abnormalities at the behavioural and at the neurophysiological level, were investigated in patients with psychotic disorder and unaffected first-degree relatives of patients with 
psychotic disorder. By including unconfounded at risk populations, we aimed to further elucidate the underlying cognitive mechanisms that play a part in psychotic symptom formation.

\section{Cognitive speed as an intermediate phenotype of psychosis and its interaction with environmental influences}

The study in chapter 2 examined in a general population female twin sample whether cognitive speed can be interpreted as a valid marker of genetic risk for psychosis and, second, whether increased genetic risk (or impaired cognitive speed performance) moderates the association between childhood trauma and positive psychotic symptoms. A cross-twin, cross-trait design that typically examines the association between two traits in twin pairs was used: one trait was measured in twin 1 (the proband twin), and another trait was measured in twin 2 (the co-twin). Because one trait can be contaminated with the other trait in the same person, this design allows the examination of the relationship in an unconfounded fashion. A total of 165 monozygotic (MZ) twin pairs and 110 dizygotic (DZ) twin pairs from the general population were included in the study. The inclusion of the DZ twin pairs allowed us to assess whether cognitive speed can be validly interpreted as marker of genetic risk by comparing the association between positive psychotic symptoms (in the proband twin) and cognitive speed (in the co-twin) between MZ and DZ twin pairs. Cognitive speed was objectively measured with three neurocognitive tests measuring speed of information processing (Stroop Colour-Word Test, Concept Shifting test and the Letter Digit Substitution test). The factor 'cognitive speed' was created from previous principal component analysis in an earlier study of our group in the same twin sample (Simons, et al., 2007). Positive subclinical psychotic symptoms were assessed with the Community Assessment of Psychic Experiences (CAPE) questionnaire (Stefanis, et al., 2002), and childhood trauma was measured using a short version of the Childhood Trauma Questionnaire (Arntz \& Wessel, 1996; Bernstein, et al., 1994).

The first question of the study was whether cognitive speed can be interpreted as a genetic risk marker of positive psychotic symptoms. Using the cross-twin, cross-trait design, it was examined whether the association between cognitive speed (assessed in the co-twin) and positive psychotic symptoms (assessed in the proband twin) was stronger in $M Z$ twins than in DZ twins. The reason to measure cognitive speed in the co-twin was to avoid a possible contamination of the outcome measure with this variable when it is 
measured in the same person. The interpretation of cognitive speed as a genetic risk marker was found to be valid because the association was only significant in MZ twins who share all of their genetic material and not in DZ twins who share only part of their genetic material. In line with previous studies (Dickinson, et al., 2007; Jabben, et al., 2007), this result confirms the assumption that cognitive speed can be seen as a useful and robust intermediate phenotype of psychosis.

The second question of the study was whether genetic risk associated with psychosis increases the association between self-reported childhood trauma and psychotic symptom formation in adulthood. Childhood trauma is one of the environmental risk factors that possibly contributes to the development of clinical and nonclinical psychotic symptoms. However, although most of the studies support the role of childhood trauma in the development of psychotic symptoms (Bebbington, et al., 2004; Janssen, et al., 2004; Read, et al., 2005; Spauwen, et al., 2006), it is less clear why not every individual who experiences a traumatic event develops psychotic symptoms. The risk-increasing effect of childhood trauma must operate in conjunction with other background vulnerability factors. It is possible that childhood trauma, as a relevant environmental risk factor, may contribute interactively with genetic factors in the development of psychotic symptom formation (van Os, et al., 2005). This assumption would imply that the association between childhood trauma and psychosis outcome is more apparent in individuals with an established vulnerability to psychosis. This question was investigated in the second part of chapter 2 in $\mathrm{MZ}$ twins. Using the cross-twin, cross-trait design, it was examined whether the association between childhood trauma and positive psychotic symptoms as the outcome measure, as assessed in the proband twin, was moderated through cognitive speed (as an indicator of genetic risk), as assessed in the co-twin. The results confirmed the assumption of a gene-adversity interaction in the formation of positive psychotic symptoms. More specifically, it was found that the association between childhood trauma and positive psychotic symptoms increased with a higher level of genetic risk (expressed as low cognitive speed performance). A similar approach was followed by Weiser and colleagues (2007), who found that the association between urbanisation and a later risk for psychotic disorder was increased by poor social and cognitive functioning, which was defined as an expression of genetic risk (Weiser, et al., 2007).

Gene-adversity interactions were reported in the context of other psychiatric phenotypes, e.g., by Caspi and colleagues (2002), who found that a functional polymorphism of the 
MAOA (monoamine oxidase A) gene was found to modify the influence of maltreated children in developing antisocial behaviour (Caspi, et al., 2002). Cognitive speed as an intermediate phenotype, or as a proxy of genetic risk, could possibly assist the search for specific genetic variations associated with this gene-adversity interaction. For example, the COMT (catechol-O-methyltransferase) gene could be a possible candidate, as the COMT Val ${ }^{158}$ Met polymorphism was found to be associated with cognitive performance in patients with psychotic disorder (Bilder, et al., 2002) and possibly moderates the risk for psychosis in terms of the interaction with environmental influences.

\section{Subjective cognitive failures as a risk factor for negative symptoms of psychosis: Individual rather than familial liability}

Cognitive impairment is usually assessed with objective measurements in the laboratory (i.e., test performance); however, a subjective estimation of one's own cognitive capacities may also be essential for our understanding of the mechanisms of symptom formation in psychosis. This point is particularly important as inner experiences of cognitive deficits were found to predict symptomatic outcome in patients with psychotic disorder (Bechdolf, et al., 2002; Klosterkotter, et al., 1997; Moritz, et al., 2000).

The frequency of subjective cognitive failures, which are cognitively based mistakes on simple tasks that can normally be achieved without failure (e.g., throwing out a new pen and keeping the old one), can be reliably measured with the self-report Cognitive Failure Questionnaire (CFQ). In a longitudinal approach, the relationship between the experience of subjective cognitive failures at baseline and subclinical psychotic symptoms at follow-up within and between genetically related individuals was examined in a family study of the general population (Chapter 3). An additional advantage of the study was the use of two valid instruments to assess subclinical psychotic symptoms in the general population: the CAPE questionnaire (measuring the frequency of and distress associated with subclinical psychotic symptoms), administered at baseline and follow-up, and the Structured Interview for Schizotypy-Revised (SIS-R) (measuring the frequency of subclinical psychotic symptoms), administered at follow-up. At baseline, the CFQ and the CAPE were administered in 755 individuals. All participants and their family members who scored high ( $>75^{\text {th }}$ percentile) or average (between $40^{\text {th }}$ and $60^{\text {th }}$ percentile) on the CAPE at baseline were asked to participate in the follow-up measurement and were reinterviewed with the CAPE $(n=501)$ and the SIS-R $(n=488)$ at follow-up. 
First, a cross-trait, within-relative approach showed that proneness to baseline subjective cognitive failures increases the risk of developing both self-reported and interview-based negative psychotic symptoms in individuals at follow-up. This association was not only found for the frequency of negative psychotic symptoms but also for the distress associated with these negative symptoms. This result suggests that not only the mere experience of the negative psychotic symptoms but also the distress associated with the subclinical psychotic experience, are affected when individuals were prone to subjective cognitive failures. The distress associated with subclinical psychotic symptoms is an important issue, as earlier studies have indicated that the transition from the subclinical to the clinical state is more related to the distress associated with the subclinical psychotic experience as the experience itself (Krabbendam, et al., 2005a; Morrison, 2001).

In line with previous studies reporting an association between high levels of the CFQ with anxiety and depression (Merckelbach, et al., 1996; Power, 1988), subjective cognitive failures were found to be associated with the frequency of self-reported depressive symptoms; however, this association did not apply to the distress associated with depressive symptoms. Furthermore, the association between subjective cognitive failures and negative and depressive symptoms was independent of the presence of these subclinical symptoms at the baseline measurement. Therefore, it can be assumed that proneness to subjective cognitive failures contributes to the development or persistence of negative or depressive psychotic symptoms and can thus be interpreted as risk factor for psychosis.

Second, the cross-trait, between-relative analyses showed no association between subjective cognitive failures in one relative and subclinical psychotic symptoms measured in the other relative. These results support an individual rather than a familial vulnerability in the development of psychotic symptoms when the experience of subjective cognitive failures is increased. Psychological mechanisms may possibly account for the association between subjective cognitive failures and subclinical psychotic symptoms. For example, it could be possible that individuals with higher levels of subjective cognitive failures try to avoid social situations where the probability of experiencing a cognitive failure is increased. As a result of this avoidance behaviour, negative symptoms may possibly develop. 
In sum, the findings from the first part of this thesis suggests that objective cognitive disturbances, such as the speed of information processing (Chapter 2), can be validly interpreted as genetic risk markers of positive psychotic experiences in $M Z$ twins and interacts with environmental risk (childhood trauma) in the development of positive psychotic symptoms. The results of the study in chapter 3 showed that the experience of subjective cognitive disturbances, such as subjective cognitive failures, can be interpreted as a potential risk factor for negative symptoms of psychosis in individuals but did not show familial continuity between the two phenotypes at the level of the general population. These findings parallel studies investigating neurocognitive test performance in relatives of patients with psychosis (Grove, et al., 1991; Nuechterlein, et al., 2002) and in individuals with a psychometrically determined proneness for psychosis (Dinn, et al., 2002; Krabbendam, et al., 2005b), showing that impaired cognition cosegregates with higher levels of subclinical psychotic symptoms. There is evidence from recent studies that the co-occurrence of these two phenotypes has a common familial basis (Krabbendam, et al., 2005b; Simons, et al., 2007). This assumption was supported by the study described in chapter 2, whereas the finding of the study in chapter 3 did not point to a shared genetic basis. These results may point to relatively independent constructs of subjective cognitive failures and objective neurocognitive test performance, which is in line with previous studies (Rabbitt \& Abson, 1990). However, it should be noted that the studies described in chapters 2 and 3 vary in respect to their sample population (MZ twin pairs versus family members of the general population). In chapter 2 the cross-sibling relationship between cognitive speed and positive psychotic symptoms was found in $\mathrm{MZ}$ twins only. This may suggest that such associations only emerge between individuals with a strong genetic relationship.

In general, cognitive impairment has been found to be more strongly correlated with the negative rather than the positive symptom dimension, both in patients with psychotic disorder and in first-degree family members (Dominguez, et al., 2009; Grove, et al., 1991). Subjective cognitive failures were expected to be more strongly related with the positive symptom dimension, as the concept of cognitive failures reflects a vulnerability to automatic intrusions that interfere with conscious processes (Power, 1988), similar to positive psychotic symptoms. However, the results of the studies in chapters 2 and 3 are not necessarily discordant with these earlier assumptions, as the correlations between the separate psychotic symptom dimensions are much higher in subclinical samples of the 
general population than in clinical samples (Stefanis, et al., 2002), thus leading to more overlap between the dimensions. A possible explanation for this finding is that the expression of the symptom dimensions is much more attenuated in the general population.

\section{Semantic network system as a specific neurocognitive intermediate phenotype of psychosis: Heterogeneous results regarding familial liability}

Abnormalities in the associative connections between words and concepts in the semantic network, resulting in intrusions of inappropriate semantic associations between words, have been found to characterise language dysfunctions in patients with psychotic disorder (Docherty, et al., 1996). Particularly, disorganized speech in patients with psychotic disorder may be related to abnormalities in the semantic memory network system. Abnormal priming effects have been detected at the behavioural level (Kerns \& Berenbaum, 2000; Moritz, et al., 1999; Moritz, et al., 2001; Spitzer, 1997) and at the neurophysiological level (Kreher, et al., 2008; Mathalon, et al., 2010a; Niznikiewicz, et al., 2004), which is consistent with broader or increased spreading activation. These impairments were also found in groups at high risk for psychosis, albeit to a lesser degree than in clinically diagnosed patients (Guerra, et al., 2009; Moritz, et al., 1999; Niznikiewicz, et al., 2004). Thus, the semantic network system of psychosis might be another possible, more specific intermediate phenotype of psychosis. The studies described in chapter 4 and chapter 5 investigated how a semantic network abnormality may be associated with the mechanism of psychotic symptom formation and whether the semantic network can be seen as an intermediate phenotype of psychosis.

The study in chapter 4 used a behavioural paradigm, the Picture-Word Interference (PWI) task, to investigate semantic priming and semantic interference effects in speech production. In the PWI-task, participants were successively presented with a prime word and a target picture. The target picture must be named while the prime word is ignored. The time course of the events (the Stimulus Onset Asynchrony, SOA) and the relationship between the prime-target pair (associative or categorical relation) were important parameters that lead to priming or interference effects within this paradigm. Priming effects can be observed if two stimuli are associated with each other because they occur frequently together in speech but do not belong to the same category (e.g., cow - milk). In contrast, interference effects can be observed if two stimuli belong to the same category 
(e.g., cow - dog) without being associatively related. Categorical interference effects are already observable at short SOA, whereas associative priming effects develop with slightly longer SOAs. In the study of chapter 4, semantic priming and interference effects were investigated in eighteen patients with psychotic disorder, twenty-five siblings of patients with psychotic disorder and eighteen healthy controls. Semantic strength was manipulated with associatively, indirectly and categorically related picture-word pairs. To reveal the time course of the priming and interference effect, three SOAs (short (-114 ms), medium (-234 ms), and long (-414 ms)) were used. Patients showed a stronger categorical interference effect at long SOAs, whereas controls did not show any interference of the categorically related prime. In contrast, siblings did not show any differences compared to the control group in any condition. These findings provide evidence for an abnormal semantic network in psychosis, showing increased interference effects in patients with psychotic disorder. However, this abnormality does not represent trait familial liability of psychosis, given a lack of group differences between siblings and healthy controls.

Semantic priming, in relation to the psychosis phenotype and as a possible liability marker of psychosis, was examined at the neurophysiological level in chapter 5 . The N400 was investigated as a relevant event-related potential (ERP) component in language processing. The $\mathrm{N} 400$ component is characterised by a negative deflection that develops around $400 \mathrm{~ms}$ after a word-stimulus is presented, and it is sensitive to the expectancy of a word in a given context, with unexpected words producing a larger (more negative) N400 component than expected words (Kutas \& Hillyard, 1980). A Lexical Decision Task was used in the study of chapter 5 . In this task, a prime word is followed by a target word and the participants had to indicate by a button press whether the target word is a real word or a nonword. The N400 was investigated after stimulus presentation of the targetword in a Lexical decision Task in twenty-two patients with a psychotic disorder, twenty siblings of patients with a psychotic disorder and twenty healthy controls. The impact of the semantic association between prime and target was controlled with associative, indirect and unrelated prime-target relations. Two SOAs (short $(-250 \mathrm{~ms})$ and long ($500 \mathrm{~ms})$ ) were used to assess the time course of automatic to more controlled processes of semantic priming. By inspecting the amplitude and the latency of the N400 component, the associative and the indirect semantic priming effect were investigated. Patients with a psychotic disorder showed an indirect priming effect of the N400 latency at automatic conditions, whereas controls did not show an indirect priming effect. In line with theories 
of a hyperactive semantic network system in psychosis (Manschreck, et al., 1988; Moritz, et al., 2002; Spitzer, 1997), these results indicate abnormal spreading of activation in the semantic network in patients with a psychotic disorder. A similar indirect priming effect of the N400 latency was also observed in the siblings, albeit statistically insignificant. However, the direction of indirect priming of the N400 latency in siblings was similar to the patient group (both showing indirect priming effects) and in clear contrast to the control group (showing no priming effect). A cautious interpretation of this indirect priming $\mathrm{N} 400$ latency effect in siblings may be that a minor increase of spreading activation may represent a possible familial vulnerability to psychotic disorder. Thus, these results provide tentative support for the $\mathrm{N400}$ as a marker for psychosis liability, representing increased semantic network activation in a high risk group.

In summary, the findings from the second part of this thesis suggest that patients with a psychotic disorder have abnormalities of the semantic network system at the behavioural (Chapter 4) and at the neurophysiological level (Chapter 5). Abnormalities of the semantic network system may reflect familial liability at the neurophysiological level (by using a Lexical Decision Task, tapping speech comprehension processes) but not at the behavioural level (by using a PWI-task, tapping speech production processes). Previous studies showed abnormalities of the semantic network system in individuals with a high risk of psychosis at the behavioural level (Moritz, et al., 1999) and at the neurophysiological level (Guerra, et al., 2009; Niznikiewicz, et al., 2002). However, other investigators have reported only very specific task-related semantic processing deficits (in a semantic categorisation task) in high schizotypy, with more intact global semantic processing (Morgan, et al., 2009), and unaffected semantic priming in individuals with high schizotypy (Morgan, et al., 2006). To date, the results of the studies in chapters 4 and 5 do not yield robust evidence for the semantic network as a candidate intermediate phenotype of psychosis, as we did not find clear abnormalities of the semantic network system in siblings of patients with psychosis.

Further, similar to previous semantic priming studies (Kiang, et al., 2008; Mathalon, et al., 2010b), the studies of chapters 4 and 5 found no relationship between semantic processing and the disorganized psychotic symptom dimension. This result possibly indicates a relative independence between abnormalities in cognitive mechanisms of the semantic network system and the disorganized symptom dimension. Another possibility 
could be that the assessment of the psychotic symptomatology, with the Brief Psychiatric Rating Scale (BPRS) in both studies, was not suitable for picking up all features of disorganized thinking in patients with psychosis.

\section{How is cognitive impairment associated with specific symptom formation?}

Although the present thesis suggests that cognitive impairment is associated with psychotic symptoms in the general population, in patients with psychotic disorder and, albeit less robustly, in first-degree family members, the results do not point to much specificity in the symptom - cognition relationship. Cognitive speed was found to be a valid marker of genetic risk in relation to the positive subclinical psychotic dimension (Chapter 2). In chapter 3, proneness to subjective cognitive failures was found to contribute to the development of negative and, to a lesser extent, to the depressive symptom domain, and this finding was only the case within rather than between siblings. The results of chapters 4 and 5 showed an abnormality of the semantic network system in patients with psychotic disorder but not specifically in relation to the disorganized dimension, which would have been plausible given that the notion of semantic network abnormalities in psychosis is meant to explain disorganized symptoms in speech. Therefore, this thesis does not provide much support for the hypothesis that cognitive impairment is linked with specific psychosis psychopathology. This may suggest that cognition, as measured in this thesis, reflects a relatively independent domain of impairment in psychosis, rather than being causally implicated in the aetiology of specific symptoms. This conclusion may also fit with the notion that there is a common underlying aetiology of all diagnostic categories of psychotic disorders (as currently categorised in the DSM-IV), as it was shown that shared genetic causes underlie the separate diagnosis in the psychotic disorder category (Cardno, et al., 2002; Kendler \& Diehl, 1993; van Os \& Kapur, 2009), suggesting more quantitative variation of the symptom dimensions, rather than qualitative differences. This being said, it should also be noted that this conclusion is partly based on findings in the general population, where a lack of specificity may be partly due to the high correlations between the separate psychotic symptom dimensions (Stefanis, et al., 2002). 


\section{Implications for clinical practice}

The results of the present thesis suggest that cognitive deficits are not only observable in patients with a psychotic disorder but can also be measured in the general population and, albeit less robustly, in first-degree relatives of patients with psychosis. Although, this vulnerability to psychosis can be measured at the subclinical level, preventive strategies in the general population are not useful, as screening for psychosis on the basis of these experiences in the general population lacks sensitivity and specificity (Van Os \& Delespaul, 2005). However, the present findings do lead to several clinical recommendations.

First, our findings indicate that cognitive deficits possibly form a relatively independent domain of impairment in psychosis. Therefore, clinical attention should be given to the cognitive deficits in patients with psychotic disorder as an independent impairment of the disorder and not only as side effect that fluctuates with the symptoms of psychosis. Thus, cognitive deficits in patients with psychotic disorder should be treated, as previous research has found that functional outcome is more strongly related to stable illness characteristics such as cognitive impairment and to a lesser extent to the more fluctuating positive symptoms of psychosis (Green, et al., 2004).

Second, informing patients about cognitive abnormalities as part of the psychosis phenotype could increase the adherence to antipsychotic medication because patients often attribute their cognitive problems to medication usage only (Ascher-Svanum, et al., 2006).

Finally, the evidence that psychotic symptoms occur not only in patients with psychotic disorders but also in individuals from the general population, albeit to a lesser degree, may help to decrease the extent of stigmatisation of psychotic disorders and mental disorders in general (Gonzalez-Torres, et al., 2007).

\section{Recommendations for further research}

The use of intermediate phenotypes, which are measurable, biologically based traits, may guide genetic research, as intermediate phenotypes are possibly closer to alterations in gene function than the psychosis phenotype. However, genetic risk alone is less likely to have a direct effect on the psychosis phenotype but genetic factors are more likely to influence the developing brain system in interactions with environmental factors (van Os \& Kapur, 2009). Therefore, intermediate phenotypes are not only useful in understanding the genetic basis of psychosis (Braff, et al., 2007) but also as broadly defined proxy 
measures of the genetic risk for psychosis in studies investigating gene-environment interactions. This strategy may be fruitful because the identification of the molecular genetic variations associated with psychosis is still in an exploratory phase (van Os \& Murray, 2008). Using this approach, Weiser et al. (2007) reported that poor premorbid social and cognitive abilities, as a proxy of genetic risk, increased the association between urbanicity and hospitalisation for schizophrenia in men (Weiser, et al., 2007). For future research, the incorporation of environmental factors in genetic risk studies, will give a broader picture of the underlying aetiology of psychosis than the investigation of intermediate phenotypes in isolation.

This thesis could not support an association between cognitive impairment and a specific symptom dimension of psychosis. The question arises whether the assessment protocols of subclinical and clinical symptoms were sensitive enough to pick up all of the aspects of the separate psychosis dimensions that are related to cognitive dysfunction. This implies that psychosis-specific assessment protocols merit further research. Finally, establishing the separate areas of cognition (objective measurable cognition, subjective experience of cognition and more specifically defined cognition) among the different psychosis dimensions in one design may possibly provide a clearer picture about the relationship between cognition and the aetiology of symptom formation in psychosis. 
Figure 1. Cognition as intermediate phenotype of psychosis in relation to predisposing genes and the psychosis phenotype.

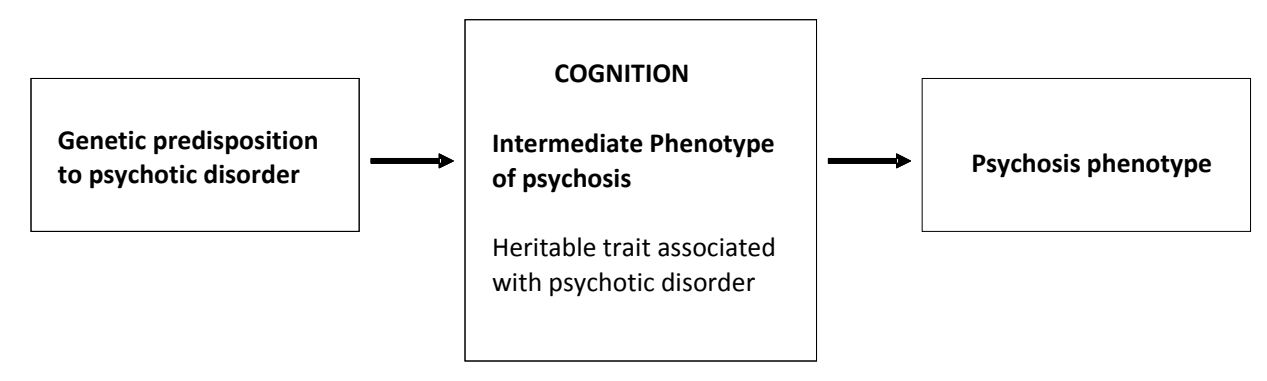




\section{References}

Arntz, A. \& Wessel, I. (1996). Jeugd Trauma Vragenlijst (Dutch version of the Childhood Trauma Questionnaire). Maastricht.

Ascher-Svanum, H., Zhu, B., Faries, D., Lacro, J. P. \& Dolder, C. R. (2006). A prospective study of risk factors for nonadherence with antipsychotic medication in the treatment of schizophrenia. J Clin Psychiatry, 67(7), 1114-1123.

Bebbington, P. E., Bhugra, D., Brugha, T., Singleton, N., Farrell, M., Jenkins, R., et al. (2004). Psychosis, victimisation and childhood disadvantage: evidence from the second British National Survey of Psychiatric Morbidity. Br J Psychiatry, 185, 220-226.

Bechdolf, A., Schultze-Lutter, F. \& Klosterkotter, J. (2002). Self-experienced vulnerability, prodromal symptoms and coping strategies preceding schizophrenic and depressive relapses. Eur Psychiatry, 17(7), 384-393.

Bernstein, D. P., Fink, L., Handelsman, L., Foote, J., Lovejoy, M., Wenzel, K., et al. (1994). Initial reliability and validity of a new retrospective measure of child abuse and neglect. Am J Psychiatry, 151(8), 1132 1136.

Bilder, R. M., Volavka, J., Czobor, P. á., Malhotra, A. K., Kennedy, J. L., Ni, X., et al. (2002). Neurocognitive correlates of the COMT Val158Met polymorphism in chronic schizophrenia. Biol Psychiatry, 52(7), 701-707.

Braff, D. L., Freedman, R., Schork, N. J. \& Gottesman, II (2007). Deconstructing schizophrenia: an overview of the use of endophenotypes in order to understand a complex disorder. Schizophr Bull, 33(1), 21-32.

Cardno, A. G., Rijsdijk, F. V., Sham, P. C., Murray, R. M. \& McGuffin, P. (2002). A twin study of genetic relationships between psychotic symptoms. Am J Psychiatry, 159(4), 539-545.

Caspi, A., McClay, J., Moffitt, T. E., Mill, J., Martin, J., Craig, I. W., et al. (2002). Role of genotype in the cycle of violence in maltreated children. Science, 297(5582), 851-854.

Dickinson, D., Ramsey, M. E. \& Gold, J. M. (2007). Overlooking the obvious: a meta-analytic comparison of digit symbol coding tasks and other cognitive measures in schizophrenia. Arch Gen Psychiatry, 64(5), 532542.

Dinn, W. M., Harris, C. L., Aycicegi, A., Greene, P. \& Andover, M. S. (2002). Positive and negative schizotypy in a student sample: neurocognitive and clinical correlates. Schizophr Res, 56(1-2), 171-185.

Docherty, N. M., DeRosa, M. \& Andreasen, N. C. (1996). Communication disturbances in schizophrenia and mania. Arch Gen Psychiatry, 53(4), 358-364.

Dominguez, M. G., Viechtbauer, W., Simons, C. J., van Os, J. \& Krabbendam, L. (2009). Are psychotic psychopathology and neurocognition orthogonal? A systematic review of their associations. Psychol Bull, 135(1), 157-171.

Gonzalez-Torres, M. A., Oraa, R., Aristegui, M., Fernandez-Rivas, A. \& Guimon, J. (2007). Stigma and discrimination towards people with schizophrenia and their family members. A qualitative study with focus groups. Soc Psychiatry Psychiatr Epidemiol, 42(1), 14-23.

Gottesman, II \& Gould, T. D. (2003). The endophenotype concept in psychiatry: etymology and strategic intentions. Am J Psychiatry, 160(4), 636-645.

Green, M. F., Kern, R. S. \& Heaton, R. K. (2004). Longitudinal studies of cognition and functional outcome in schizophrenia: implications for MATRICS. Schizophr Res, 72(1), 41-51.

Grove, W. M., Lebow, B. S., Clementz, B. A., Cerri, A., Medus, C. \& lacono, W. G. (1991). Familial prevalence and coaggregation of schizotypy indicators: a multitrait family study. J Abnorm Psychol, 100(2), 115-121.

Guerra, S., Ibáñez, A., Martín, M., Bobes, M. A., Reyes, A., Mendoza, R., et al. (2009). N400 deficits from semantic matching of pictures in probands and first-degree relatives from multiplex schizophrenia families. Brain Cogn, 70(2), 221-230.

Heinrichs, R. W. \& Zakzanis, K. K. (1998). Neurocognitive deficit in schizophrenia: a quantitative review of the evidence. Neuropsychology, 12(3), 426-445.

Hughes, C., Kumari, V., Das, M., Zachariah, E., Ettinger, U., Sumich, A., et al. (2005). Cognitive functioning in siblings discordant for schizophrenia. Acta Psychiatr Scand, 111(3), 185-192.

Jabben, N., van Os, J., Janssen, I., Versmissen, D. \& Krabbendam, L. (2007). Cognitive alterations in groups at risk for psychosis: neutral markers of genetic risk or indicators of social disability? Acta Psychiatr Scand, 116(4), 253-262.

Janssen, I., Krabbendam, L., Bak, M., Hanssen, M., Vollebergh, W., de Graaf, R., et al. (2004). Childhood abuse as a risk factor for psychotic experiences. Acta Psychiatr Scand, 109(1), 38-45.

Keefe, R. S., Bilder, R. M., Harvey, P. D., Davis, S. M., Palmer, B. W., Gold, J. M., et al. (2006). Baseline neurocognitive deficits in the CATIE schizophrenia trial. Neuropsychopharmacol, 31(9), 2033-2046. 
Kendler, K. S. \& Diehl, S. R. (1993). The genetics of schizophrenia: a current, genetic-epidemiologic perspective. Schizophr Bull, 19(2), 261-285.

Kerns, J. G. \& Berenbaum, H. (2000). Aberrant semantic and affective processing in people at risk for psychosis. J Abnorm Psychol, 109(4), 728-732.

Kiang, M., Kutas, M., Light, G. A. \& Braff, D. L. (2008). An Event-Related Brain Potential Study of Direct and Indirect Semantic Priming in Schizophrenia. Am J Psychiatry, 165(1), 74-81.

Klosterkotter, J., Schultze-Lutter, F., Gross, G., Huber, G. \& Steinmeyer, E. M. (1997). Early self-experienced neuropsychological deficits and subsequent schizophrenic diseases: An 8-year average follow-up prospective study. Acta Psychiatr Scand, 95(5), 396-404.

Krabbendam, L., Marcelis, M., Delespaul, P., Jolles, J. \& van Os, J. (2001). Single or multiple familial cognitive risk factors in schizophrenia? Am J Med Genet, 105(2), 183-188.

Krabbendam, L., Myin-Germeys, I., Hanssen, M., de Graaf, R., Vollebergh, W., Bak, M., et al. (2005a). Development of depressed mood predicts onset of psychotic disorder in individuals who report hallucinatory experiences. Br J Clin Psychol, 44(Pt 1), 113-125.

Krabbendam, L., Myin-Germeys, I., Hanssen, M. \& van Os, J. (2005b). Familial covariation of the subclinical psychosis phenotype and verbal fluency in the general population. Schizophr Res, 74(1), 37-41.

Kreher, D. A., Holcomb, P. J., Goff, D. \& Kuperberg, G. R. (2008). Neural Evidence for Faster and Further Automatic Spreading Activation in Schizophrenic Thought Disorder. Schizophr Bull, 34(3), 473-482.

Kremen, W. S., Seidman, L. J., Pepple, J. R., Lyons, M. J., Tsuang, M. T. \& Faraone, S. V. (1994). Neuropsychological risk indicators for schizophrenia: a review of family studies. Schizophr Bull, 20(1), 103-119.

Kutas, M. \& Hillyard, S. A. (1980). Reading senseless sentences: brain potentials reflect semantic incongruity. Science, 207(4427), 203-205.

Manschreck, T. C., Maher, B. A., Milavetz, J. J., Ames, D., Weisstein, C. C. \& Schneyer, M. L. (1988). Semantic priming in thought disordered schizophrenic patients. Schizophr Res, 1(1), 61-66.

Mathalon, D. H., Roach, B. J. \& Ford, J. M. (2010a). Automatic semantic priming abnormalities in schizophrenia. Int J Psychophysiol, 75(2), 157-166.

Mathalon, D. H., Roach, B. J. \& Ford, J. M. (2010b). Automatic semantic priming abnormalities in schizophrenia. Int J Psychophysiol, 75(2), 157-166.

Merckelbach, H., Muris, P., Nijman, H. \& de Jong, P. J. (1996). Self-reported cognitive failures and neurotic symptomatoloy. Pers Indiv Differ, 20(6), 715-724.

Morgan, C., Bedford, N. \& Rossell, S. L. (2006). Evidence of semantic disorganisation using semantic priming in individuals with high schizotypy. Schizophr Res, 84(2-3), 272-280.

Morgan, C. J., Bedford, N. J., O'Regan, A. \& Rossell, S. L. (2009). Is semantic processing impaired in individuals with high schizotypy? J Nerv Ment Dis, 197(4), 232-238.

Moritz, S., Andresen, B., Domin, F., Martin, T., Probsthein, E., Kretschmer, G., et al. (1999). Increased automatic spreading activation in healthy subjects with elevated scores in a scale assessing schizophrenic language disturbances. Psychol Med, 29(1), 161-170.

Moritz, S., Krausz, M., Gottwalz, E., Lambert, M., Perro, C., Ganzer, S., et al. (2000). Cognitive dysfunction at baseline predicts symptomatic 1-year outcome in first-episode schizophrenics. Psychopathology, 33(1), 48-51.

Moritz, S., Mersmann, K., Kloss, M., Jacobsen, D., Wilke, U., Andresen, B., et al. (2001). 'Hyper-priming' in thought-disordered schizophrenic patients. Psychol Med, 31(2), 221-229.

Moritz, S., Woodward, T. S., Kuppers, D., Lausen, A. \& Schickel, M. (2002). Increased automatic spreading of activation in thought-disordered schizophrenic patients. Schizophr Res, 59(2-3), 181-186.

Morrison, A. P. (2001). The interpretation of intrusions in psychosis: An integrative cognitive approach to hallucinations and delusions. Behav and Cogn Psychother, 29(3), 257-276.

Niznikiewicz, M. A., Friedman, M., Shenton, M. E., Voglmaier, M., Nestor, P. G., Frumin, M., et al. (2004). Processing sentence context in women with schizotypal personality disorder: An ERP study. Psychophysiology, 41(3), 367-371.

Niznikiewicz, M. A., Shenton, M. E., Voglmaier, M., Nestor, P. G., Dickey, C. C., Frumin, M., et al. (2002). Semantic dysfunction in women with schizotypal personality disorder. Am J Psychiatry, 159(10), 1767-1774.

Nuechterlein, K. H., Asarnow, R. F., Subotnik, K. L., Fogelson, D. L., Payne, D. L., Kendler, K. S., et al. (2002). The structure of schizotypy: relationships between neurocognitive and personality disorder features in relatives of schizophrenic patients in the UCLA Family Study. Schizophrenia Research, 54(1-2), 121130.

Power, M. J. (1988). Cognitive failures, dysfunctional attitudes, and symptomatology: A longitudinal study. Cog Emo, 2(2), 133-143. 
Rabbitt, P. \& Abson, V. (1990). 'Lost and found': some logical and methodological limitations of self-report questionnaires as tools to study cognitive ageing. Br J Psychol, 81(Pt 1), 1-16.

Read, J., van Os, J., Morrison, A. P. \& Ross, C. A. (2005). Childhood trauma, psychosis and schizophrenia: a literature review with theoretical and clinical implications. Acta Psychiatr Scand, 112(5), 330-350.

Simons, C. J., Jacobs, N., Jolles, J., van Os, J. \& Krabbendam, L. (2007). Subclinical psychotic experiences and cognitive functioning as a bivariate phenotype for genetic studies in the general population. Schizophr Res, 92(1-3), 24-31.

Sitskoorn, M. M., Aleman, A., Ebisch, S. J., Appels, M. C. \& Kahn, R. S. (2004). Cognitive deficits in relatives of patients with schizophrenia: a meta-analysis. Schizophr Res, 71(2-3), 285-295.

Spauwen, J., Krabbendam, L., Lieb, R., Wittchen, H. U. \& van Os, J. (2006). Impact of psychological trauma on the development of psychotic symptoms: relationship with psychosis proneness. Br J Psychiatry, 188, 527533.

Spitzer, M. (1997). A cognitive neuroscience view of schizophrenic thought disorder. Schizophr Bull, 23(1), 29-50.

Stefanis, N. C., Hanssen, M., Smirnis, N. K., Avramopoulos, D. A., Evdokimidis, I. K., Stefanis, C. N., et al. (2002). Evidence that three dimensions of psychosis have a distribution in the general population. Psychol Med, 32(2), 347-358.

Van Os, J. \& Delespaul, P. (2005). Toward a world consensus on prevention of schizophrenia. Dialogues Clin Neurosci, 7(1), 53-67.

van Os, J. \& Kapur, S. (2009). Schizophrenia. Lancet, 374(9690), 635-645.

van Os, J., Krabbendam, L., Myin-Germeys, I. \& Delespaul, P. (2005). The schizophrenia envirome. Curr Opin Psychiatry, 18(2), 141-145.

van Os, J. \& Murray, R. (2008). Introduction. Schizophr Bull, 34(6), 1064-1065.

VogImaier, M. M., Seidman, L. J., Salisbury, D. \& McCarley, R. W. (1997). Neuropsychological dysfunction in schizotypal personality disorder: a profile analysis. Biol Psychiatry, 41(5), 530-540.

Weiser, M., van Os, J., Reichenberg, A., Rabinowitz, J., Nahon, D., Kravitz, E., et al. (2007). Social and cognitive functioning, urbanicity and risk for schizophrenia. Br J Psychiatry, 191, 320-324. 



\section{SUMMARY}

The presence of cognitive impairments is not only a core feature of psychotic disorders but alterations in cognitive mechanisms can also be found in individuals with a higher than average risk to develop psychosis, suggesting that cognitive impairments form a vulnerability marker for psychosis. This thesis entitled 'At risk for psychosis: Exploring cognition as intermediate phenotype' examines cognition as intermediate phenotype, how dysfunction in cognitive processes is related to symptom formation of the broader psychosis phenotype and how it interacts with environmental influences.

Chapter 1 provides an introduction to the phenomenology and the aetiology of psychosis. The search for causal mechanisms of psychosis has led to an increased interest in the study of intermediate phenotypes, which are measurable and trait-related characteristics, indicating a covert link between a predisposition to psychosis and the psychosis phenotype. Cognitive functioning has been found to be a promising indicator of psychosis liability. The chapter gives an overview of the evidence for cognition as intermediate phenotype of psychosis. Cognitive speed, as an example of objective measurable cognition, is presented as a promising cognitive intermediate phenotype, and it is suggested that it may interact with environmental influences in the development of psychosis. Subjective cognitive experiences are discussed in the context of psychotic symptom formation and familial liability. Finally, the semantic network system is presented in relation to symptom formation of psychosis and in the context of a more specific neurocognitive intermediate phenotype of psychosis.

Chapter 2 examines the effect of cognitive impairment as a genetic risk marker on the association between childhood trauma and positive psychotic symptom formation in a general population twin study. A total of 165 monozygotic (MZ) twin pairs and 110 dizygotic (DZ) twin pairs from the general population were included in the study. Cognitive impairment was assessed with a composite measure reflecting cognitive speed. Positive subclinical psychotic symptoms were assessed with the Community Assessment of Psychic Experiences Questionnaire and childhood trauma was measured using a short version of 
the Childhood Trauma Questionnaire. First, it was established whether cognitive speed can be validly interpreted as a genetic risk marker of positive psychotic symptoms, and whether it should thus be considered an intermediate phenotype of psychosis. By using a cross-twin, cross-trait design, this assumption was confirmed by the finding that the link between subclinical psychotic symptoms (measured in the proband twin) and cognitive speed (measured in the co-twin) was much stronger in $M Z$ twin pairs compared to $D Z$ twin- pairs. Second, it was investigated whether cognitive speed, as a genetic risk marker, moderates the impact of self-reported childhood trauma on the development of psychotic symptoms in $\mathrm{MZ}$ twins. It was shown that the risk increasing effect of childhood trauma in the development of positive psychotic symptoms in later life in the proband twin was increased with a decreased level of cognitive speed in the co-twin, indicating that the experience of traumatic events in childhood is particularly negative when cognitive functioning is impaired as part of the genetic liability of psychosis.

Chapter 3 examines whether proneness to subjective cognitive failures, as measured with the self-report Cognitive Failure Questionnaire (CFQ), contributes to the development or persistence of subclinical psychotic symptoms, as assessed with the self-report Community Assessment of Psychic Experiences (CAPE) questionnaire and the Structured Interview for Schizotypy-Revised (SIS-R). The CFQ and the CAPE were assessed at the baseline measurement $(n=755)$. Individuals scoring high $\left(>75^{\text {th }}\right.$ percentile) or average (between $40^{\text {th }}$ and $60^{\text {th }}$ percentile) on the CAPE were reinterviewed with the CAPE $(n=501)$ and the SIS-R $(n=488)$ at follow-up. The association between subjective cognitive failures and subclinical psychotic symptoms was investigated within and between genetically related individuals of the general population. First, it was shown that within individuals, proneness to baseline subjective cognitive failures increases the risk of follow-up negative (self-report and interview based) and depressive (only self-report) psychotic symptoms but not of positive psychotic symptoms. Moreover, this association was independent of the presence of negative or depressive symptoms at the baseline measurement. These results showed that proneness to subjective cognitive failures increases the risk for the development of negative or depressive symptoms of psychosis. Second, the betweenrelative analyses showed no association between subjective cognitive failures in one relative and subclinical psychotic symptom measured in the other relative, indicating that there is no direct genetic transmission of the two phenotypes. The results thus suggest 
that the experience of subjective cognitive failures may be a risk factor for the development of negative and depressive symptoms in individuals but the overlap between the cognitive and symptom domains do not have a familial basis.

Chapter 4 reports a study investigating semantic priming and interference effects in speech production in 18 patients with a psychotic disorder, 25 siblings of patients with a psychotic disorder and 18 healthy controls. A picture-word interference task was used in which participants were presented with a to-be-named target picture and an accompanying prime word which must be ignored. Reaction times were measured by verbal naming latencies of the picture onset until the participants gave a response. Semantic strength was varied by using categorically (based on the same semantic category of prime and target, e.g., wolf - lion), associatively (based on the meaning relationship of the prime and target, e.g., glasses - eye) and indirectly (associatively related but the association between prime and target is only obvious through a mediating associated word, e.g., lemon - (sour) - sweet) related picture-word pairs. Three different Stimulus Onset Asynchronies (SOAs; short (- $114 \mathrm{~ms})$, medium (-234 ms), and long (-414 ms)) were used to reveal the time course of the priming and interference effect. The results showed a stronger interference effect in patients with a psychotic disorder compared to the siblings and the healthy control group only at the long SOA. In contrast to prior reports of increased associative and indirect spreading of activation in patients with a psychotic disorder, patients did not show enhanced semantic priming effects compared to firstdegree relatives or controls at any SOA. Further, the present study failed to find any significantly altered semantic priming and interference effects in siblings. These findings demonstrate that an abnormal semantic network is associated with psychotic disorder but does not represent trait familial liability.

Chapter $\mathbf{5}$ examines semantic priming effects in 22 patients with a psychotic disorder, 20 siblings of patients with a psychotic disorder and 20 healthy controls at a neurophysiological level. A Lexical Decision Task was used in which a prime word was followed by a target word, and the participants had to indicate by a button press whether the target word is a real word or a nonword. Event-related potentials (ERPs) were recorded to target words that were associatively related (based on a meaning relationship of the prime and target, e.g., glasses - eye), indirectly related (associatively related but the 
association between prime and target is only obvious through a mediating associated word, e.g., lemon - (sour) - sweet) and un-related (no relation of prime and target, e.g., car - table) to their preceding prime word. Latency and amplitude of the N400 ERP component were measured as a probe of semantic processing. The $\mathrm{N} 400$ component is a negative deflection around $400 \mathrm{~ms}$ after target onset, which develops in response to any meaningful stimulus. To assess the time course of automatic to more controlled processes of semantic priming, two Stimulus Onset Asynchronies (SOAs; short (-250ms) and long ($500 \mathrm{~ms})$ ) were investigated. Under automatic experimental conditions, patients with a psychotic disorder showed indirect priming of the $\mathrm{N} 400$ latency, supporting the assumption of a hyperactive semantic network in patients with a psychotic disorder, whereas indirect N400 amplitude did not differ between the three groups. There was no increase in the associative priming effect in patients with psychotic disorder, neither at N400 latency nor at the N400 amplitude. The results in siblings mimicked the indirect N400 latency priming effect found in the patient group, suggesting that abnormal spreading activation represents a possible familial liability to psychotic disorder.

Chapter 6 presents a summary of the main findings of the thesis. This chapter discusses cognition as intermediate phenotype, its relationship to symptom formation of the broader psychosis phenotype and its interaction with environmental influences. Finally, clinical recommendations and directions for further research are given. 




\section{Samenvatting}

Het optreden van cognitieve stoornissen is niet alleen een hoofdkenmerk van patiënten met een psychotische stoornis, maar veranderingen in cognitieve mechanismen worden ook gevonden in individuen met een verhoogd risico op het ontwikkelen van psychotische klachten. Dit duidt erop dat cognitie als een marker voor kwetsbaarheid voor psychose kan worden gezien. Dit proefschrift getiteld 'At risk for psychosis: Exploring cognition as intermediate phenotype' onderzoekt cognitie als intermediair fenotype, hoe dysfuncties van cognitieve processen zich verhouden tot symptoom formatie van het bredere psychosefenotype en hoe deze cognitieve intermediaire fenotypen zich verhouden in interactie met de omgeving.

Hoofdstuk 1 geeft een introductie betreffende de fenomenologie en etiologie van psychose. Het zoeken naar causale mechanismen van psychose heeft geleid tot een toenemende interesse in het bestuderen van intermediaire fenotypen. Intermediaire fenotypen zijn meetbare eigenschappen, die op een predispositie voor psychose wijzen. Cognitief functioneren wordt beschouwd als een veelbelovend intermediair fenotype. Snelheid van informatieverwerking is een voorbeeld van objectief meetbare cognitie. Eerder onderzoek heeft aangetoond dat snelheid van informatieverwerking een veelbelovend intermediaire fenotype is en mogelijk interacteert met de omgeving tot het ontstaan van psychotische symptomen. Vervolgens worden subjectieve cognitieve ervaringen binnen de context van ontwikkeling van psychotische symptomen en familiare kwetsbaarheid voor psychose besproken. Daarnaast wordt het semantische netwerk als model voor psychotische symptomen en als mogelijk specifiek intermediair fenotype voor psychose beschreven.

Hoofdstuk 2 onderzocht het effect van cognitie als genetische risicomarker en de invloed op de associatie van trauma in de kindertijd en positieve psychotische symptomen. Dit wordt onderzocht in een algemene populatie steekproef van vrouwelijke tweelingparen (165 monozygote en 110 dizygote tweelingparen). Snelheid van informatieverwerking werd bepaald met een samengestelde meetbatterij van cognitieve testen, positieve 
symptomen op subklinisch niveau werden met de CAPE vragenlijst (Community Assessment of Psychic Experiences) gemeten en trauma in de kindertijd met een korte versie van de CTQ (Childhood Trauma Questionnaire). Ten eerste wordt in dit hoofdstuk vastgesteld of snelheid van informatieverwerking als genetische risicomarker van positieve psychotische symptomen kan worden geïnterpreteerd en daarmee als intermediaire fenotype kan worden gezien. Deze assumptie werd bevestigd middels een cross-twin, cross-trait onderzoeksopzet: positieve symptomen op subklinisch niveau (gemeten in tweeling 1) en snelheid van informatieverwerking (gemeten in tweeling 2) waren sterker geassocieerd in monozygote tweelingen dan in dizygote tweelingen. Ten tweede wordt onderzocht of snelheid van informatieverwerking (als genetisch risicomarker) een modererende invloed heeft op de associatie van trauma in de kindertijd en psychotische symptomen in monozygote tweelingen. Uit de resultaten bleek dat het effect van trauma in de kindertijd op het ontstaan van positieve psychotische symptomen later in het leven in tweeling 1 was toegenomen met een afnemende prestatie van informatiesnelheid in tweeling 2. Deze bevindingen geven aan dat het meemaken van een traumatische gebeurtenis in de kindertijd voornamelijk negatieve consequenties heeft bij een verhoogde genetische kwetsbaarheid voor psychose.

Hoofdstuk 3 onderzoekt het optreden van subjectieve cognitieve fouten, gemeten met de Cognitive Failure Questionnaire (CFQ) in relatie tot het ontwikkelen of persisteren van subklinische psychotische ervaringen. Subklinische psychotische ervaringen worden gemeten met de CAPE zelfrapportage vragenlijst en de Structured Interview for Schizotypy-Revised (SIS-R). De CFQ en de CAPE waren op de baseline meting afgenomen $(n=755)$. Proefpersonen die hoog $\left(>75^{\text {ste }}\right.$ percentiel) of gemiddeld (tussen $40^{\text {ste }}$ en $60^{\text {ste }}$ percentiel) op de CAPE scoorden, werden nogmaals geïnterviewd met de CAPE ( $n=501)$ en de SIS-R ( $n=488$ ) op de follow-up meting. De associatie tussen subjectieve cognitieve fouten en subklinische psychotische symptomen werd binnen en tussen genetischgerelateerde personen van de algemene populatie onderzocht. Ten eerste werd aangetoond dat binnen personen met een neiging tot subjectieve cognitieve fouten het risico toeneemt om negatieve (zelfrapportage en met interview gemeten) en depressieve (enkel zelfrapportage) psychotische symptomen op follow-up te laten zien. Deze associatie was onafhankelijk van het hebben van negatieve of depressieve symptomen op de baseline meting. Deze bevindingen geven aan dat een neiging tot subjectieve 
cognitieve fouten het risico laat toenemen om negatieve of depressieve symptomen van psychose te ontwikkelen. Ten tweede heeft de analyse tussen familieleden geen associatie laten zien van subjectieve cognitieve fouten in een familielid en subklinische psychotische symptomen in het andere familielid. Dit toont aan dat er geen directe genetische transmissie van deze twee fenotypen is. Deze resultaten suggereren dat de ervaring van subjectieve cognitieve fouten mogelijk een risicofactor is voor de ontwikkeling van negatieve en depressieve symptomen, maar dat er onder de overlap tussen het cognitieve en het symptoomdomein geen familiaire basis ligt.

Hoofdstuk 4 rapporteert een studie die semantische priming- en interferentie-effecten in spraakproductie in 18 patiënten met een psychotische stoornis, 25 broers/zussen van patiënten met een psychotische stoornis en 18 gezonde controles onderzoekt. Een plaatje-woord interferentietaak (Picture-Word Interference task) wordt gebruikt, waarin proefpersonen een targetplaatje (wat ze moesten benoemen) en een bijgaand primewoord (wat ze moesten negeren) te zien krijgen. Reactietijden worden bepaald door het meten van de tijd van de beginpresentatie van het plaatje tot het moment waarop de proefpersoon een verbale respons heeft gegeven. Semantische sterkte wordt gevarieerd door categorisch (gebaseerd op dezelfde semantische categorie van prime en target, bijv. wolf- leeuw), associatief (gebaseerd op de relatie van betekenis van prime en target, bijv. bril - oog) en indirect (associatief gerelateerd, maar de associatie tussen prime en target is alleen zichtbaar door een niet weergegeven verbindingswoord, bijv. citroen - (zuur) zoet) gerelateerde plaatje-woord paren te gebruiken. Drie verschillende stimulus onset asynchronies (SOAs; kort (- $114 \mathrm{~ms})$, medium (-234 ms) en lang (-414 ms)) werden gebruikt om het tijdsverloop van het priming- en interferentie-effect te verduidelijken. De bevindingen lieten een sterker interferentie-effect zien na een lange SOA in patiënten met een psychotische stoornis vergeleken met de broers/zussen en gezonde controles. In vergelijking met eerdere studies die toegenomen priming-effecten in patiënten met een psychotische stoornis hebben aangetoond vergeleken met gezonde controles, lieten patiënten in de huidige studie geen toegenomen semantische priming-effecten zien vergeleken met de andere groepen. De huidige studie liet verder geen significante veranderingen in semantische priming en interferentie in broers/zussen zien. Deze bevindingen demonstreren dat een abnormaal semantisch netwerk geassocieerd is met psychotische stoornissen, maar geen familiaire kwetsbaarheid representeert. 
Hoofdstuk $\mathbf{5}$ onderzocht semantische priming-effecten op neurofysiologisch niveau in 22 patiënten met een psychotisch stoornis, 20 broers/zussen van patiënten met een psychotische stoornis en 20 gezonde controles. Een lexicale beslissingstaak wordt gebruikt, waarbinnen een primewoord wordt gepresenteerd, gevolgd door een targetwoord. De proefpersonen moeten middels een druk op de knop aangeven of het target woord een echt woord of een non-woord is. Event-gerelateerde potentialen (ERPs) worden opgenomen. De prime-target relaties waren associatief (gebaseerd op de relatie van betekenis van prime en target, bijv. bril - oog), indirect (associatief gerelateerd, maar de associatie tussen prime en target is alleen zichtbaar door een niet weergegeven verbindingswoord, bijv. citroen - (zuur) - zoet) en niet-gerelateerde (geen relatie van prime en target, bijv. auto- tafel). Latentie en amplitude van de N400 ERP-component worden gemeten. De N400 is een negatieve buiging ongeveer $400 \mathrm{~ms}$ na de onset van de target en ontwikkelt zich als antwoord op elke betekenisvolle stimulus. Om het tijdsverloop van automatische naar meer gecontroleerde processen van semantische priming te onderzoeken, zijn twee SOAs (kort $(-250 \mathrm{~ms})$ en lang $(-500 \mathrm{~ms})$ ) gebruikt. De resultaten lieten zien dat patiënten met een psychotische stoornis indirecte priming van de N400 latentie alleen onder automatische condities lieten zien. Dit ondersteunt de assumptie van een hyperactief semantisch netwerk in patiënten met een psychotische stoornis. De indirecte priming op de $\mathrm{N} 400$ amplitude liet geen verschil tussen de drie groepen zien. De huidige studie kon echter geen toenemend associatieve priming-effect in patiënten met een psychotische stoornis aantonen, noch voor de N400 latentie, noch voor de N400 amplitude. De bevindingen in broers/zussen zijn identiek aan de indirecte N400 latentie priming-effect die in de patiëntgroep was gevonden. Dit suggereert dat abnormale spreiding van activiteit een mogelijke familiare kwetsbaarheidsmarker voor psychotisch stoornis is.

Hoofdstuk 6 presenteert een samenvatting van de belangrijkste bevindingen van dit proefschrift. Het hoofdstuk illustreert cognitie as een intermediair fenotype, hoe dysfuncties van cognitieve processen zich verhouden tot symptoomformatie van het bredere psychosefenotype en hoe deze cognitieve intermediaire fenotypen zich in interactie met de omgeving verhouden. Het hoofdstuk besluit met klinische implicaties en suggesties voor toekomstig onderzoek. 




\section{Dankwoord}

Graag wil ik een woord van dank aan al die mensen richten die een bijdrage hebben geleverd aan de totstandkoming van dit proefschrift.

Allereerst gaat mijn dank uit naar mijn promotor, Prof. dr. Jim van Os en mijn copromotor Dr. Lydia Krabbendam. Beste Jim, dank je wel dat ik bij jou mocht leren en werken, en bedankt voor al je kennis, inspiratie en gedrevenheid en voor al je optimisme dat je overbrengt op alle AIOs en mensen met wie je samenwerkt. Beste Lydia, dank je wel dat ik de kans heb gekregen om van jouw deskundigheid te kunnen leren, voor de altijd prettige samenwerking en de vrijheid die ik in denken en handelen van jou heb gekregen. En voor het altijd warme welkom bij jouw thuis.

Ook wil ik mijn tweede promotor, Prof. dr. Niels Schiller en Prof. dr. Wim Riedel bedanken voor de samenwerking bij de priming studies. Bedankt voor de interesse in de vooruitgang van de studies, het overleg als er technische problemen waren en de feedback op de manuscripten van de studies. Verder wil ik Prof. dr. Inez Myin-Germeys bedanken. Inez, jouw gedrevenheid, enthousiasme en de altijd prettige gesprekken hebben vooral in de laatste jaren mijn AiO-periode tot een heel plezierige tijd gemaakt. Door jou kon ik altijd weer de (onderzoeks)-wereld in een breder licht zien.

Ook wil ik alle proefpersonen bedanken die hebben meegewerkt aan Geestkracht, en in het bijzonder de proefpersonen die bereid waren om urenlang achter een scherm te zitten om niet de aller-interessantste taken uit te voeren. Zonder hulp van een aantal stagiaires en onderzoeksassistenten was de dataverzameling van deze studie niet tot stand gekomen: Ellen, Karlijn, Crispijn en Ilse, bedankt voor jullie ondersteuning.

Tevens wil ik alle coauteurs bedanken voor de prettige samenwerking en voor de gelegenheid die ik in de afgelopen jaren heb gekregen om met de hele mooie COMED en TWIN dataset te mogen werken. Ook wil ik alle leden van de leescommissie en corona bedanken. 
Tineke en Christine, mijn paranimfen, jullie van harte bedankt dat jullie op die dag aan mijn kant staan. Tineke, mijn kamergenoot, bedankt voor de gezellige praatjes, het brainstormen en de gezellige tijd op congressen. Christine, Danke für die interessanten Gespräche, dein immer offenes Ohr und die ganzen Kuchen- und Reiswaffelkrümel die wir in unseren Wohnzimmern produziert haben.

Ook wil ik de andere collega's van SP en KP bedanken. ledereen heeft eraan bijgedragen dat ik het altijd prettig en gezellig vond om hier te werken en dat ik de sfeer van SP ontzettend ga missen. In het bijzonder wil ik alle onderzoeksassistenten en stagiaires bedanken voor jullie hulp bij de dataverzameling. Truda, Inge, Frida, Miriam en Debora, bedankt voor de ondersteuning bij het project en de data-invoer. Ron, bedankt voor het altijd snelle oplossen van alle problemen die een computer met zich meebrengt. Trees, Jolanda, Ine en Leni, bedankt voor de administratieve ondersteuning en het zorgen dat er altijd iets lekkers voor de medewerkers was. En natuurlijk alle AIOs voor de fijne samenwerking en al die gezelligheid in de laatste jaren. Ik had me geen betere werkplek kunnen wensen.

Marijntje, Claudia, Odette en Viviane, jullie waren geweldige kamergenoten. Bedankt voor de mooie tijd.

Verder wil ik Dr. Franz Wojciechowski en het team van de eetpoli in het AZM bedanken dat ik de kans bij jullie heb gekregen om klinische ervaring op te doen.

Bedanken möchte ich mich auch bei Prof. Dr. Bernadette Jansma. Bernadette, durch dich habe ich wissenschaftliches Arbeiten schon im Studium kennen gelernt und meine Freude an der Forschung entdeckt. Danke, dass ich so viel von Dir lernen konnte und Du es mir ermöglicht hast bei SP als AIO anzufangen. Ook wil ik Mart en Petra van harte bedanken. Door jullie enthousiasme in het doen van onderzoek gepaard met jullie optimisme heb ik onderzoek doen op een heel fijne manier leren kennen. Bedankt ook dat ik altijd bij jullie mocht aankloppen. Petra, bedankt voor de ondersteuning van het EEG project.

Liebe Freunde und Familie, von Herzen vielen Dank für die wunderschöne Zeit in Aachen/Maastricht/Augsburg. Miri, Karen, Johanna und Christine meine liebsten 
Weggefährten, ich werde euch schrecklich vermissen. Cecile, bedankt voor al het lachen over de onderzoeks- en familiewereld. Zonder jou was het werk half zo plezierig geweest.

Babsi, von Herzen Danke für Deine Hilfe, Unterstützung, Fröhlichkeit, Deinen unglaublichen Optimismus und Deine Fähigkeit alles wieder ins Lot zu rücken. Du bist die beste Schwester auf der ganzen Welt. Und Deinen Jungs, Kili und Lorenz, meine allerliebsten Neffen und weltbesten Cousins, ich freue mich schon auf eure roten Wangen beim stundenlangen Wii spielen, wenn ihr mit euren Nasen vor dem Bildschirm klebt.

Liebe Mama, lieber Papa, es gibt keine Worte für Eure Liebe und Unterstützung, Euer Vertrauen in mich und für alle Chancen und Möglichkeiten die Ihr mir gegeben habt. Von Herzen Danke für alles.

Andi und Elea, Ihr seid für mich das größte Glück auf Erden. Danke. Und für Elea: einen dicken Kuss von Deiner Mama. 



\section{Curriculum vitae}

Stefanie Pfeifer was born on October 11, 1975 in Augsburg, Germany. After finishing secondary school in 1994 (Fachoberschule Augsburg), she studied nursing in Augsburg (Zentralklinikum Augsburg), finished her training in 1998 and worked for one year at the same hospital. Thereafter, she studied three semester social pedagogy at the University of Applied Science in Koblenz, Germany. In October 2001, she started studying Psychology at Maastricht University. She graduated in August 2005 and received her master's degree in Biological Psychology (Biological Developmental Psychology). In October 2005, she started working as a PhD student at the Department of Psychiatry and Neuropsychology, School for Mental Health and Neuroscience, Maastricht University, where she investigated cognitive intermediate phenotypes in psychosis. In her dissertation project she was involved as researcher in the Genetic Risk and Outcome of Psychosis study (www.groupproject.nl), a nationwide study investigating vulnerability and protective factors in psychosis.

Currently, she is a postdoctoral fellow at the Department of Psychiatry and Neuropsychology, Maastricht University. Stefanie Pfeifer is married to Andreas Zimmerer and together they have a one year old daughter, Elea. 



\section{List of publications}

\section{International journals}

Pfeifer S, van Os J, Hanssen M, Delespaul P \& Krabbendam L (2009). Subjective experience of cognitive failures as possible risk factor for negative symptoms of psychosis in the general population. Schizophrenia Bulletin, 35(4): 766-774.

Pfeifer S, Krabbendam L, Myin-Germeys I, Derom C, Wichers M, Thiery E \& van Os J (2010). A cognitive endophenotype study confirming possible gene-early adversity interaction in psychosis outcome: A general population twin study. Psychosis, 2(1): 1-11.

Pfeifer S, Krabbendam L \& van Os J (2010). Psychosis, 2(3): 268-269.

\section{Submitted papers}

Pfeifer S, Schiller NO, van Os J, Riedel JW, Simons C \& Krabbendam L. Semantic activation in patients with a psychotic disorder and first-degree relatives. Submitted.

Pfeifer S, Schiller NO, van Os J, Riedel JW, Simons C, Vlamings P \& Krabbendam L. Electrophysiological correlates of automatic spreading of activation in patients with psychotic disorder and first-degree relatives. Submitted.

Simons C, Sambeth A, van Os J, Krabbendam L, Pfeifer S \& Riedel WJ. Auditory P300 and N100 components as intermediate phenotypes for psychotic disorder: familial liability and reliability. Submitted.

Heins M, van Os J, Lataster T, Simons C, Pfeifer S, Versmissen D, Lardinois M, Habets P, Krabbendam L \& Myin-Germeys I. Childhood trauma and psychosis: Evidence from a large epidemiological sample. Submitted. 


\section{Conference presentations}

Pfeifer S, van Os J, Hanssen M \& Krabbendam L (2007). Subjective Experience of Cognitive Failures predicts subclinical negative symptoms in the General Population. Schizophrenia Bulletin, 33 (2): 244.

Pfeifer S, Schiller NO, van Os J \& Krabbendam L (2008). Abnormale semantische Aktivierung bei Patienten mit psychotischer Störung und deren Geschwistern: Eine BildWort Interferenz Studie. DGPPN Kongress Berlin, 2008: 72.

Pfeifer S, Krabbendam L, Myin-Germeys I, Derom C \& van Os J (2008). A cognitive endophenotype study confirming possible gene-early adversity interaction in psychosis outcome. Schizophrenia Research, 102 (1-3), Supplement 2: 125.

Pfeifer S, van Os J, Hanssen M, Delespaul P \& Krabbendam L (2008). Subjectieve ervaring van cognitieve fouten als potentieel risico factor voor psychotische symptomen in de algemene populatie. DePsychiater, 15 (1): 40.

Pfeifer S, Schiller NO, van Os J, Riedel JW \& Krabbendam L (2009). Semantic Priming abnormalities as putative endophenotype of psychosis: an event-related potential study. Schizophrenia Bulletin, 35 (1): 248.

Pfeifer S, Schiller NO, van Os J, Riedel JW, Simons C, Vlamings P \& Krabbendam L (2010). Abnormal activation of semantic networks as intermediate phenotype for psychotic disorder. Early Intervention in Psychiatry, $7^{\text {th }}$ International conference onEarly Psychosis, Amsterdam, 2010, 4, Supplement 1: 42. 

